\title{
DIRECT INTEGRAL DECOMPOSITIONS AND MULTIPLICITIES FOR INDUCED REPRESENTATIONS OF NILPOTENT LIE GROUPS
}

\author{
L. CORWIN, F. P. GREENLEAF AND G. GRELAUD
}

\begin{abstract}
Let $K$ be a Lie subgroup of the connected, simply connected nilpotent Lie group $G$, and let $\mathfrak{k}, \mathfrak{g}$ be the corresponding Lie algebras. Suppose that $\sigma$ is an irreducible unitary representation of $K$. We give an explicit direct integral decomposition of $\operatorname{Ind}_{k \rightarrow G} \sigma$ into irreducibles. The description uses the Kirillov orbit picture, which gives a bijection between $G^{\wedge}$ and the coadjoint orbits in $\mathfrak{g}^{*}$ (and similarly for $\left.K^{\wedge}, \mathfrak{f}^{*}\right)$. Let $P: \mathfrak{1}^{*} \rightarrow \mathrm{g}^{*}$ be the canonical projection, let $\mathcal{O}_{\mathrm{o}} \subset \mathfrak{k}^{*}$ be the orbit corresponding to $\sigma$, and, for $\pi \in G^{\wedge}$, let $\mathcal{O}_{\pi} \subset \mathrm{g}^{*}$ be the corresponding orbit. The main result of the paper says essentially that $\pi \in G^{\wedge}$ appears in the direct integral iff $P^{-1}\left(\mathcal{O}_{\sigma}\right)$ meets $\mathcal{O}_{\pi}$; the multiplicity of $\pi$ is the number of $\operatorname{Ad}^{*}(K)$-orbits in $\mathcal{O}_{\pi} \cap P^{-1}\left(\mathcal{O}_{\sigma}\right)$. There is also a natural description of the measure class in the integral.
\end{abstract}

1. Let $G$ be a connected, simply connected, nilpotent Lie group, with Lie algebra $\mathrm{g}$, let $\mathfrak{f}$ be a subalgebra of $\mathrm{g}$, and let $K$ be the corresponding subgroup of $G$. Given an irreducible unitary representation $\chi$ of $K$, let $\rho=\operatorname{Ind}_{K \rightarrow G} \chi$ be the unitary representation of $G$ induced from $\chi$. Kirillov theory associates an $\operatorname{Ad}^{*}(K)$-orbit $\mathcal{O}_{\chi} \subseteq \mathfrak{f}^{*}$ with $\chi$. Denote by $P$ the canonical projection of $\mathrm{g}^{*}$ on $\mathfrak{H}^{*}$. In his fundamental paper [9], Kirillov remarks that $\rho$ is a direct integral of the irreducible unitary representations of $G$ whose corresponding orbits meet $P^{-1}\left(\mathcal{O}_{\chi}\right)$. This statement is rather vague, since it fails to address the question of multiplicity. It is shown in [7] that $\rho$ is quasi-equivalent to the direct integral (with respect to an appropriate measure) of the set of representations $\pi \in G^{\wedge}$ such that $\mathcal{O}_{\chi}$ meets $\rho^{-1}\left(\mathcal{O}_{\sigma}\right)$. However, $\rho$ need not be multiplicity-free, or even of uniform multiplicity. (We shall give examples in §7.) More, then, is needed for a complete description of $\rho$. In fact, it is possible to use the Kirillov orbital picture to give this description, as we shall show.

We will give a direct integral decomposition of $\rho=\operatorname{Ind}_{K \rightarrow G}(\chi)$ in which both the base space and the multiplicities are explicitly computed in terms of the geometry of orbits. For this we employ the theory of semialgebraic sets, introduced by Tarski and Seidenberg [14, 12]. If $V \cong \mathbf{R}^{n}$, a set $S \subseteq V$ is semialgebraic if it is determined by a finite number of polynomial equalities $p_{i}(v)=0$ and inequalities $p_{i}(v)>0$ (where $p_{i}$ are polynomials over $\mathbf{R}$ ), or if it is a Boolean combination of such sets and their complements. If $S$ is semialgebraic it has a stratification $\mathscr{P}$, a partition $S=S_{1}$ $\cup \cdots \cup S_{m}(m<\infty)$ such that

(i) Each $S_{i}$ is a connected, embedded submanifold in $V$ (manifold topology = relative topology).

Received by the editors November 21, 1986.

1980 Mathematics Subject Classification (1985 Revision). Primary 22E27.

(C)1987 American Mathematical Society $0002-9947 / 87 \$ 1.00+\$ .25$ per page 
(ii) For all $x \in V$, there exists a neighborhood $N_{x} \subseteq V$ such that $N_{x} \cap S_{i}$ is connected (or empty), for $i=1,2, \ldots, m$.

(iii) $S_{i} \cap S_{j}^{-} \neq \varnothing \Rightarrow S_{i} \subseteq S_{j}^{-}$.

(iv) Each $S_{i}$ is semialgebraic.

The main properties we need are:

(v) If $f: V \rightarrow V$ is a polynomial map (or is rational, nonsingular on $S$ ) then $f(S$ ) is semialgebraic.

This is one of the main results of Tarski-Seidenberg, see [14].

(vi) If $\mathscr{P}_{1}, \mathscr{P}_{2}$ are stratifications of $S$, there is a stratification $\mathscr{P}$ that is a refinement of both.

Let $k=k(S)=\max \left\{\operatorname{dim} S_{i}: 1 \leqslant i \leqslant m\right\}$ for a stratification of $S$. This is independent of the stratification, by (vi). For any $\mathscr{P}$ we can define a measure $\nu_{\mathscr{P}}$ on $S$ by taking a nonvanishing $k$-dimensional volume on each $k$-dimensional piece of $S$. Various stratifications of $S$ give the same measure class [ $\nu$ ]. If $A \subseteq S$ is semialgebraic and dense in $S$, then $\operatorname{dim}(S \backslash A)<\operatorname{dim} S$, and $\nu(S \backslash A)=0$.

A set is algebraic if it is the difference of Zariski-open sets, i.e. the intersection of a Zariski-closed set with a Zariski-open set. The spectrum of the induced representation may lie anywhere in $G^{\wedge}$, and does not necessarily consist of representations in general position. Thus we need a cross-section for all $\mathrm{Ad}^{*}(G)$-orbits in $\mathrm{g}^{*}$. Following Pukanszky [10], one can always partition $\mathrm{g}^{*}$ into $\mathrm{Ad}^{*}(G)$-invariant layers $U_{e^{(1)}} \cup \cdots \cup U_{e^{(r)}}$ such that each $U_{e}$ is a computable algebraic set and has a computable cross-section $\Sigma_{e}$, also an algebraic set. $\mathrm{Ad}^{*}(G)$-orbits in each layer $U_{e}$ all have the same dimension. The set $\Sigma=\bigcup_{i=1}^{r} \sum_{e^{(i)}}$ is then a semialgebraic set cross-sectioning all orbits in $\mathrm{g}^{*}$. We will show that the layers may be chosen so that $W_{i}=$ (union of the first $i$ layers) is a Zariski-open set in $\mathrm{g}^{*}$ for $1 \leqslant i \leqslant r$; in particular, the set $U_{e^{(1)}}$ is Zariski-open, and constitutes the generic orbits.

Now $P^{-1}\left(\mathcal{O}_{\chi}\right)$ is an irreducible algebraic variety, since this is true of $\mathcal{O}_{\chi} \subseteq \mathbb{F}^{*}$. Therefore, if $U_{e}=U_{e^{(i)}}$ is the first layer that meets $P^{-1}\left(\mathcal{O}_{\chi}\right)$, the intersection $U_{e} \cap P^{-1}\left(\mathcal{O}_{\chi}\right)=W_{i} \cap P^{-1}\left(\mathcal{O}_{\chi}\right)$ is Zariski-open in this variety. Let $\Sigma^{\chi}=\Sigma_{e} \cap$ $\operatorname{Ad}^{*}(G) P^{-1}\left(O_{\chi}\right)$ be the orbit representatives for this intersection. It is not hard to see that $\Sigma^{\chi}$ is a semialgebraic set, and so determines a unique measure class $\left(\Sigma^{x}, \nu\right)$. This class is the base space for the direct integral decomposition.

In terms of Zariski-open sets in the variety $P^{-1}\left(\mathcal{O}_{\chi}\right)$, we can define the following parameters without reference to orbit cross-sections. Noting that $P^{-1}\left(\mathcal{O}_{\chi}\right)$ is $\operatorname{Ad}^{*}(K)$-invariant, because $\mathcal{O}_{\chi}$ is a $K$-orbit in $\mathfrak{F}^{*}$, we let

$r=$ generic (maximal) dimension of an $\mathrm{Ad}^{*}(K)$-orbit in $P^{-1}\left(\mathcal{O}_{\chi}\right)$,

$s=$ generic value of $\operatorname{dim} \mathcal{O}_{l}$ for $\operatorname{Ad}^{*}(G)$-orbits $\mathcal{O}_{l}=\operatorname{Ad}^{*}(G) l$ in $\mathrm{g}^{*}$ that meet $P^{-1}\left(\mathcal{O}_{\chi}\right)$.

Clearly, $s=\operatorname{dim} \operatorname{Ad}^{*}(G) l$ for $l \in U_{e}$. With this definition in mind, we define the "defect" parameter

$$
\tau_{0}=s-2 r+\operatorname{dim} \mathcal{O}_{\chi}
$$

and can now state our main theorem. 
THEOREM. Let $K$ be a closed connected subgroup of a nilpotent Lie group $G$. Let $\chi \in K^{\wedge}$ correspond to the $\operatorname{Ad}^{*}(K)$-orbit $\mathcal{O}_{\chi} \subseteq \mathfrak{k}^{*}$, and let $P^{-1}\left(\mathcal{O}_{\chi}\right)$ be its liftback under the natural projection $P: \mathrm{g}^{*} \rightarrow \mathfrak{1}^{*}$. Define $r, s$, and the defect parameter $\tau_{0}$ as above. Given a strong Mal'cev basis $X_{1}, \ldots, X_{n}$ in $\mathrm{g}$, construct the layers $U_{e^{(i)}}$ and cross-sections $\Sigma_{e^{(i)}}$ in $\mathrm{g}^{*}$, determine the first layer $U_{e}$ that meets $P^{-1}\left(\mathcal{O}_{\chi}\right)$, and construct the unique measure class $\left(\Sigma^{x}, \nu\right)$ as described above.

If $\tau_{0}>0$, all multiplicities are infinite and

$$
\operatorname{Ind}_{K \rightarrow G}(\chi)=\int_{\Sigma^{x}}^{\oplus} \infty \cdot \pi_{l} d \nu(l)
$$

where $\pi_{l} \in G^{\wedge}$ is the representation associated with $l \in \mathrm{g}^{*}$.

If $\tau_{0}=0$, there is a stratification of $\Sigma^{x}$ such that the union $S=S_{1} \cup \cdots \cup S_{p}$ of its maximum dimensional pieces has the following properties:

(a) If $l \in S$, then $\mathcal{O}_{1}=\operatorname{Ad}^{*}(G)$ l meets $P^{-1}\left(\mathcal{O}_{\chi}\right)$ in a closed submanifold.

(b) If $l \in S$, the connected components of $\mathcal{O}_{1} \cap P^{-1}\left(\mathcal{O}_{\chi}\right)$ are precisely the $\operatorname{Ad}^{*}(K)$ orbits in this intersection, and are finite in number.

(c) The function $n(l)=$ number of $\operatorname{Ad}^{*}(K)$-oribts in $P^{-1}\left(\mathcal{O}_{\chi}\right) \cap \mathcal{O}_{l}$ is constant on each piece $S_{i}, 1 \leqslant i \leqslant p$. In this case we have

$$
\operatorname{Ind}_{K \rightarrow G}(\chi)=\int_{\Sigma^{x}}^{\oplus} n(l) \pi_{l} d \nu(l)=\int_{S}^{\oplus} n(l) \pi_{l} d \nu(l) .
$$

In particular, there is a uniform bound $n(l) \leqslant N$ for the multiplicities.

This description of the multiplicity of $\pi_{l}$ in the induced representation as the number of $\operatorname{Ad}^{*}(K)$-orbits in $\mathcal{O}_{1} \cap P^{-1}\left(\mathcal{O}_{\chi}\right)$ bears a striking similarity to some results in [4], where the following problem was considered: Let $\Gamma$ be a discrete, cocompact subgroup of $G$, and let $\chi_{0}$ be a 1-dimensional unitary representation of $\Gamma$. Suppose that

(a) $\Lambda=\log \Gamma \subseteq g$ is a Lie ring;

(b) $\chi_{0}$ extends to a 1-dimensional representation of $G$ (exponentiated from if $\left.{ }^{\prime}, f^{\prime} \in g^{*}\right)$.

Consider $\operatorname{Ind}_{\Gamma \rightarrow G}\left(\chi_{0}\right)=\rho_{0}$. It is a discrete direct sum of irreducible unitary representations. Which ones occur, and with what multiplicities? The answer is that $\pi$ occurs iff $\mathcal{O}_{\pi}$ meets $\Lambda^{\perp}+f^{\prime}$ and that the multiplicity is given by a formula involving the $\operatorname{Ad}^{*}(\Gamma)$-orbits in $\Lambda^{\perp}+f$. (See [5] for additional information about the constants in the formula.)

The theorem stated above was proved simultaneously and independently by the first two authors (Corwin and Greenleaf) and by the third (Grelaud). The methods used in the two approaches are quite different. The proof given here is the first of these, not because we think it is necessarily intrinsically better but because one account of the other approach is by now accessible in the literature [8]. Here is a brief sketch of the other proof, which is measure theoretic in its details. Let $\mu$ be a finite measure on $P^{-1}\left(\mathcal{O}_{\chi}\right)$ equivalent to $q$-dimensional measure, where $q=$ $\operatorname{dim} P^{-1}\left(\mathcal{O}_{\chi}\right)$. The first step is to show that $\operatorname{Ind}_{K \rightarrow G}(\chi)$ is quasi-equivalent to $\int_{P^{-1}\left(O_{\chi}\right)}^{\oplus} \pi_{l} d \mu(l)$. This is done by induction on $\operatorname{dim} G$; one can assume that, for a 
normal subgroup $G_{0}$ of codimension 1,

$$
\text { Ind }_{K \rightarrow G_{0}}(\chi) \text { quasi-equivalent to } \int_{P_{0}^{-1}\left(O_{\chi}\right)}^{\oplus} \pi_{l^{\prime}} d \mu\left(l^{\prime}\right)
$$

where $\mathrm{g}_{0}$ is the Lie algebra of $G_{0}$ and $P_{0}: \mathrm{g}_{0}^{*} \rightarrow \mathfrak{f}^{*}$ is the natural projection. In inducing from $G_{0}$ to $G$, either (a) almost all $\pi_{l}$, induce to irreducibles, or (b) almost none induce to irreducibles. One handles each of these possibilities separately.

The proof of the multiplicity formula follows a similar pattern. We may assume the result for $G_{0}$; to go to $G$ we need to analyze the component representations Ind $_{G_{0} \rightarrow G}\left(\pi_{l^{\prime}}\right)$, and must determine when the same representation $\pi_{l}$ of $G$ occurs in two (or more) different component representations. An analysis of the two possibilities mentioned above shows that in case (b) nearly all disjoint representations induce to disjoint representations, and the inductive proof is not hard. In case (a), the analysis of multiplicities is more subtle. Throughout the proof one must, of course, take into account a number of measure-theoretic complications. In our notation, the final result of this approach can be stated as follows.

THEOREM. With notation as above, let $f \in \mathcal{O}_{x} \subseteq \mathfrak{l}^{*}$ and let $f^{\prime}$ be any extension of $f$ to g. Then

$$
\operatorname{Ind}_{K \rightarrow G}(\chi)=\int_{G^{\wedge}}^{\oplus} n(\pi) d \nu^{\prime}(\pi)
$$

where $\nu^{\prime}$ is the push-forward to $G^{\wedge} \approx g^{*} / \operatorname{Ad}^{*}(G)$ of a finite measure $\mu^{\prime}$ on $f^{\prime}+\mathfrak{f}^{\perp}=$ $P^{-1}\{f\}$ which is equivalent to Lebesgue measure. When

$$
\operatorname{dim} \mathcal{O}_{l}-\operatorname{dim} \mathcal{O}_{\chi}=2 \operatorname{dim}\left(\mathcal{O}_{1} \cap\left(f^{\prime}+\mathfrak{H}^{\perp}\right)\right) \quad \text { for almost all } l \in f^{\prime}+\mathfrak{H}
$$

( with respect to $\nu^{\prime}$ ), the multiplicities are given by

$$
n(\pi)=\text { number of connected components of } \mathcal{O}_{\pi} \cap\left(f^{\prime}+\mathfrak{f}^{\perp}\right) .
$$

Otherwise, the multiplicity is

$$
n(\pi)=\infty \text { for } \nu^{\prime} \text {-almost all } \pi .
$$

As part of the proof, it is shown that $\mathcal{O}_{1} \cap\left(f^{\prime}+\mathfrak{t}^{\perp}\right)$ is a closed submanifold for $\nu^{\prime}$-almost every $l \in f^{\prime}+\mathfrak{f}^{\perp}$. It is not hard to show that this version of the multiplicity theorem is equivalent to the one stated previously (which will be proved in this paper), except that the existence of a uniform bound for the multiplicities in the finite multiplicity case seems to require the techniques from algebraic geometry used in the present account. On the other hand, the proof in [8] also applies to the case when $G$ is an exponential, solvable Lie group and $K$ is a connected normal subgroup; then $\operatorname{Ind}_{K \rightarrow G}(\chi)$ is either multiplicity-free or of uniform infinite multiplicity. In the solvable, exponential case, there is an example in which the dimension criterion for finite multiplicity stated above is valid, but nevertheless $n(\pi)=\infty$. (In this example, a spiral orbit meets a flat variety in infinitely many points; thus infinite multiplicity is no longer associated with orbit intersections having too high a dimension, as is the case with nilpotent groups.) The case of a general connected $K$ in an exponential solvable group is apparently still open; see [2] for one result, describing the spectrum only. 
Here is a brief description of our method of attack and of the organization of the paper. For most of the paper, we consider the case where $\operatorname{dim} \chi=1$. Then, of course, there is an element $f \in \mathfrak{H}^{*}$ such that $\chi(\exp Y)=\exp$ if $(Y)$ for all $Y \in \mathfrak{f}^{\prime}$. Let $f^{\prime}$ be any extension of $f$ to $g$. Then $P^{-1}\left(\mathcal{O}_{\chi}\right)=f^{\prime}+\mathfrak{f}^{\perp}$. Our first main step (in §3) is to find a subset $E \subseteq \mathfrak{f}^{\perp}$ such that

$$
\rho \cong \int_{E}^{\oplus} \pi_{l+f^{\prime}} d l
$$

here, $E$ turns out to be a Zariski-open subset of a subspace of $\mathfrak{L}^{\perp}$, and $d l$ is Lebesgue measure on the subspace. Of course, we may well have $\pi_{l_{1}+f^{\prime}} \cong \pi_{l_{2}+f^{\prime}}$ for distinct $l_{1}, l_{2} \in E$, so that (1) does not give information about multiplicities. We therefore must analyze the map $\phi: E \rightarrow \Sigma_{e}$ which maps $l \in E$ to the element $l^{\prime} \in \Sigma_{e}$ with $l+f^{\prime} \in \mathcal{O}_{l^{\prime}}$. This analysis takes up $\S \S 4$ and 5 ; it is complicated by our need to exclude sets on which the rational map $\phi$ exhibits singular behavior. In $\S 6$ we remove the hypothesis that $\chi$ be 1-dimensional, thus completing the proof. $\S 2$ is devoted to various algebraic preliminaries concerning parametrization of orbits, and $\S 7$ to a number of examples.

The reduction to the case when $P^{-1}\left(\mathcal{O}_{\chi}\right)$ is a flat variety should be no surprise. If $\chi \in K^{\wedge}$, it is induced from some character $\sigma=e^{2 \pi i f_{0}}$ on a maximal subordinate subgroup $K_{0}$. If $P_{0}: \mathrm{g}^{*} \rightarrow \mathfrak{f}_{0}^{*}$ is the natural projection and if $f^{\prime}$ is any extension of $f_{0}$ to all of $\mathrm{g}$, then $\mathcal{O}_{\sigma}=\left\{f_{0}\right\}, P_{0}^{-1}\left(\mathcal{O}_{\sigma}\right)=f^{\prime}+\mathfrak{H}_{0}^{\perp}$, and we are reduced to studying $\operatorname{Ad}^{*}\left(K_{0}\right)$-orbits in the flat variety $f^{\prime}+\mathfrak{H}_{0}^{\perp}$. More is true: $P_{0}^{-1}\left(\mathcal{O}_{\sigma}\right)$ is contained in $P^{-1}\left(\mathcal{O}_{\chi}\right)$ and we will show that, for any $l \in \mathrm{g}^{*}$, the $K$-orbits in $P^{-1}\left(\mathcal{O}_{\chi}\right) \cap \mathcal{O}_{l}$ are in one-to-one correspondence with the $K_{0}$-orbits in $\mathcal{O}_{1} \cap\left(f^{\prime}+\mathfrak{H}_{0}^{\perp}\right)$. The invariant $\tau_{0}$ has the same value in both situations, $\Sigma^{\chi}=\Sigma^{\chi_{0}}$, and we get exactly the same direct integral decomposition from either point of view.

2. We shall need three facts about coadjoint orbits in nilpotent Lie groups; at least one is well known.

Let $G$ be a connected, simply connected, nilpotent Lie group with Lie algebra $g$, and let $G_{0}$ be a connected closed subgroup of codimension 1; denote by $\mathfrak{g}_{0}$ the Lie algebra corresponding to $G_{0}$, and let $P: \mathrm{g}^{*} \rightarrow \mathrm{g}_{0}^{*}$ be the canonical projection. Given an irreducible unitary representation $\pi_{0}$ of $G_{0}$, we let $\mathcal{O}_{\pi_{0}}$ be the $\operatorname{Ad}^{*}\left(G_{0}\right)$-orbit in $\mathfrak{g}_{0}^{*}$ corresponding to $\pi_{0}$ via the Kirillov map.

Proposition 1. Let notation be as above. Then

(a) There exists at least one $\operatorname{Ad}^{*}(G)$-orbit $\mathcal{O}$ in $\mathrm{g}^{*}$ with $P(\mathcal{O}) \supseteq \mathcal{O}_{\pi_{0}}$.

(b) Either

(1) $\mathcal{O}$ is unique, in which case $P^{-1}\left(\mathcal{O}_{\pi_{0}}\right) \varsubsetneqq \mathcal{O}$ and $\operatorname{dim} \mathcal{O}=\operatorname{dim} \mathcal{O}_{\pi_{0}}+2$, or

(2) $\mathcal{O}$ is not unique, in which case $P \mathcal{O}^{\prime}=\mathcal{O}_{\pi_{0}}$ for any $\mathcal{O}^{\prime}$ with $P\left(\mathcal{O}^{\prime}\right) \supseteq \mathcal{O}_{\pi_{0}}$, and $\operatorname{dim} \mathcal{O}^{\prime}=\operatorname{dim} \mathcal{O}_{\pi_{0}}$.

(c) In case (1), the representation $\pi \in G^{\wedge}$ corresponding to $\mathcal{O}$ is induced from $\pi_{0}$. Moreover, if $l \in P^{-1}\left(\mathcal{O}_{\pi_{0}}\right)$, then $\operatorname{Ad}^{*}\left(G_{0}\right) l=P^{-1}\left(\mathcal{O}_{\pi_{0}}\right)$.

(d) In case (2), let $\mathcal{O}$ be an orbit as in (a). Then for any nonzero $l \in \mathrm{g}_{0}^{\perp}$, the orbit $\mathcal{O}^{\prime}$ satisfies $P\left(\mathcal{O}^{\prime}\right) \supseteq \mathcal{O}_{\pi_{0}}$ if and only if $\mathcal{O}^{\prime}=\mathcal{O}+\alpha l=\mathcal{O}^{(\alpha)}$ for some $\alpha \in \mathbf{R}$; the $\mathcal{O}^{(\alpha)}$ are 
disjoint. Letting $\pi^{(\alpha)} \in G^{\wedge}$ correspond to $\mathcal{O}^{(\alpha)}$, we have $\pi^{(\alpha)} \mid G_{0} \cong \pi_{0}$ and

$$
\operatorname{Ind}_{G_{0} \rightarrow G}\left(\pi_{0}\right) \cong \int_{\mathbf{R}}^{\oplus} \pi^{(\alpha)} d \alpha,
$$

or equivalently,

$$
\operatorname{Ind}_{G_{0} \rightarrow G}\left(\pi_{0}\right) \cong \int_{\mathbf{R}}^{\oplus} \pi_{\alpha l+l^{\prime}} d \alpha
$$

where $l^{\prime} \in \mathcal{O}$ is fixed.

Proof. Except for the last claim in (d), this is a summary of results proved in [10, pp. 525-527]. The claim is part of Lemma 6.2 in [9].

We now examine the way in which various sets of representations of a subgroup behave with respect to the larger group. The main result follows from some elementary algebraic geometry.

Let $G$ and $\mathrm{g}$ be as before, and let $K$ be a connected, closed subgroup with Lie algebra $\mathfrak{k}$. Form a chain of $\mathfrak{f}=\mathfrak{g}_{0} \subsetneq \mathfrak{g}_{1} \subsetneq \cdots \varsubsetneqq \mathfrak{g}_{m}=\mathfrak{g}$, with $\operatorname{dim} \mathfrak{g}_{i+1}=\operatorname{dim} \mathfrak{g}_{i}$ $+1,0 \leqslant i \leqslant m-1$. Let $G_{i}$ be the group corresponding to $\mathfrak{g}_{i}$, and let $P_{i}=\mathfrak{g}^{*} \rightarrow \mathrm{g}_{i}^{*}$ be the canonical projection.

Proposition 2. For $l \in \mathrm{g}^{*}$, let $d_{i}(l)=\operatorname{dim}\left(\operatorname{Ad}^{*} G_{i}\right)\left(P_{i}(l)\right)$, and let $d(l)=$ $\left(d_{0}(l), \ldots, d_{m}(l)\right)$. If $d=\left(d_{0}, \ldots, d_{m}\right)$ is any $m+1$ tuple, let $U_{d}=\{l: d(l)=d\}$ and let $\mathscr{D}=\left\{d: U_{d} \neq \varnothing\right\}$. Then there exists an ordering of the elements of $\mathscr{D}$, $\mathscr{D}=\left\{d^{(1)}>d^{(2)}>\cdots\right\}$ such that for each $d \in \mathscr{D}$ the set $V_{d}=\bigcup\left\{U_{d^{\prime}}: d^{\prime} \geqslant d\right\}$ is Zariski-open. In particular, each $U_{d}$ is the set-theoretic difference of two Zariski-open sets in $V$.

Proof. If $d \in \mathscr{D}$, the $d_{i}$ are increasing with $i$ and are even because all $\operatorname{Ad}^{*}\left(G_{j}\right)$ orbits in $\mathrm{g}_{j}^{*}$ have even dimension. Let $X_{1}, \ldots, X_{n}$ be a basis of $\mathrm{g}$ such that $X_{1}, \ldots, X_{n-m+j}$ is a basis of $g_{j}$ for $0 \leqslant j \leqslant m$, and let $l_{1}, \ldots, l_{n}$ be the dual basis in $\mathrm{g}^{*}$. Saying that $d_{j}(l) \geqslant q$ for some $j$ is equivalent to saying that

$$
\operatorname{dim}\left(\operatorname{span}\left\{\operatorname{ad}^{*} X_{1}(l), \ldots, \operatorname{ad}^{*} X_{n-m+j}(l)\right\}+\mathfrak{g}_{j}^{\perp}\right) \geqslant m+q-j .
$$

In coordinates, this is equivalent to saying that at least one of a certain set of determinants depending polynomially on $l$ is nonzero, which is a Zariski-open condition. Thus the set $S_{d}=\left\{l: d_{j}(l) \geqslant d_{j}\right.$, all $\left.j\right\}$ is Zariski-open in $\mathrm{g}^{*}$.

Partially order $\mathscr{D}$ by letting $d^{\prime} \succ d$ if $d_{j}^{\prime} \geqslant d_{j}$ for all $j$. Let $d_{j}^{(1)}=$ maximal dimension of $\operatorname{Ad}^{*}\left(G_{j}\right)$-orbits in $\mathfrak{g}_{j}^{*}$. Then $U_{d^{(1)}}=\bigcap_{j=0}^{m}\left\{l \in \mathrm{g}^{*}: \operatorname{dim} \operatorname{Ad}^{*}\left(G_{j}\right)\left(P_{j} l\right)=\right.$ $\left.d_{j}^{(1)}\right\}=\bigcap_{j=0}^{m}\left\{l: \operatorname{dim} \operatorname{Ad}^{*}\left(G_{j}\right)\left(P_{j} l\right) \geqslant d_{j}^{(1)}\right\}$, which is nonempty and Zariski-open; $d^{(1)}$ is the unique maximal element in $D$. Inductively define $\mathscr{D}_{1}=\left\{d^{(1)}\right\}, \mathscr{D}_{k+1}=$ $\left\{d: d\right.$ maximal in $\left.\left(\mathscr{D} \backslash \bigcup_{j \leqslant k} \mathscr{D}_{j}, \succ\right)\right\}$. Then list the elements of $\mathscr{D}$ as $d^{(1)}>d^{(2)}>$ $\cdots$ by listing successively the elements in $\mathscr{D}_{1}, \mathscr{D}_{2}, \ldots$, taking any order within a particular set $\mathscr{D}_{k}$.

For brevity denote $V_{k}=V_{d^{(k)}}, U_{k}=U_{d^{(k)}}, S_{k}=S_{d^{(k)}}$ for $d^{(k)} \in \mathscr{D}$. We claim that $V_{k}=U_{j \leqslant k} U_{j}$ is equal to $U_{j \leqslant k} S_{j}$ for each $k$; this clearly shows that the $V_{k}$ are Zariski-open, as desired. Obviously $S_{j} \supseteq U_{j}$ and $U_{j \leqslant k} S_{j} \supseteq U_{j \leqslant k} U_{j}=V_{k}$, so it suffices to show $S_{i} \subseteq V_{i}$ for each $i$, since the $V_{k}$ are increasing with $k$. Consider any 
$d^{(i)} \in \mathscr{D}_{k}=\left\{d^{(p)}, \ldots, d^{(q)}\right\}$ and let $l \in S_{i}$. Now $l \in U_{r}$ for some $r$. If $r \geqslant p$, this would imply that $d_{j}(l)=d_{j}^{(r)} \geqslant d_{j}^{(i)}$ for all $0 \leqslant j \leqslant m$, by definition of $l \in S_{i}$ and $l \in U_{r}$. This means that $d^{(r)}>d^{(i)}$ in $(\mathscr{D}, \succ)$. But by the way we have listed the $d^{(j)}$ and grouped them, $d^{(i)}$ is maximal in $\left(\mathscr{D} \backslash \bigcup_{j \leqslant k-1} \mathscr{D}_{j}\right)$. But $d^{(r)}$ is in this set if $r \geqslant p$, so in this case we would conclude that $r=i, d^{(r)}=d^{(i)}$. Therefore,

$$
l \in S_{i} \Rightarrow l \in U_{i} \cup\left(\bigcup\left\{U_{d}: d \in \bigcup_{j \leqslant k-1} \mathscr{D}_{j}\right\}\right)
$$

and

$$
S_{i} \subseteq U_{i} \cup\left(\bigcup_{j<p} U_{j}\right) \subseteq \bigcup_{j \leqslant i} U_{j}=V_{i}
$$

for any $i$. This proves the result.

Note. One can actually show directly that each $U_{d}$ is a difference of Zariski-open sets. The point of making a careful choice of ordering $d^{(1)}>d^{(2)}>\cdots$ is that the "layers" $U_{d}$ should always yield Zariski-open sets $V_{d}$ as they are adjoined successively. This property is important later on.

A variant of this result holds in the case of a chain of normal subgroups. The following theorem is certainly known in part, but there does not seem to be a convenient reference.

We let $G, \mathfrak{g}$ be as before; let $\mathfrak{g}_{1} \subseteq \mathfrak{g}_{2} \subseteq \cdots \subseteq \mathfrak{g}_{n}$ be a chain of ideals in $\mathfrak{g}$ with $\operatorname{dim} \mathfrak{g}_{j}=j$. Let $X_{1}, \ldots, X_{n}$ be a basis of $\mathfrak{g}$ such that $X_{1}, \ldots, X_{j}$ span $\mathfrak{g}_{j}(1 \leqslant j \leqslant n)$, let $l_{1}, \ldots, l_{n}$ be the dual basis in $\mathrm{g}^{*}$, and let $P_{j}: \mathrm{g}^{*} \rightarrow \mathrm{g}_{j}^{*}$ be the canonical projection. For $l \in \mathrm{g}^{*}$, let $e_{i}(l)=\operatorname{dim} P_{i}\left(\operatorname{Ad}^{*}(G) l\right)$ and let $e(l)=\left(e_{1}(l), \ldots, e_{n}(l)\right)$, a nondecreasing list of integers. (Note that $G$ acts on $G_{j}$ by conjugation, hence on $\mathfrak{g}_{j}$ and also $\mathrm{g}_{j}^{*}$; the projection $P_{j}$ is equivariant for these actions of $G$, so that $P_{j}\left(\operatorname{Ad}^{*}(G) l\right)=\operatorname{Ad}^{*}(G)\left(P_{j} l\right)$ is a manifold. Let $U_{e}^{-}=\{l: e(l)=e\}$ and $\mathscr{E}=$ $\left\{e: U_{e}^{-} \neq \varnothing\right\}$.

THEOREM 1. Let $G$ be a nilpotent Lie group and let ideals $(0)=g_{0} \subseteq g_{1} \subseteq \cdots \subseteq$ $\mathrm{g}_{n}=\mathrm{g}$ be specified as above. Then

(a) $\mathscr{E}$ is finite and $e=\left(e_{1}, \ldots, e_{n}\right) \in \mathscr{E} \Rightarrow e_{j+1}=e_{j}$ or $e_{j+1}=e_{j}+1$ for $j<n$.

(b) There is an ordering of elements of $\mathscr{E}, e^{(1)}>e^{(2)}>\cdots>e^{(k)}$ such that $\bigcup_{j \leqslant i} U_{e^{(j)}}$ is Zariski-open in $\mathrm{g}^{*}$ for each $i$. In particular, each $U_{e^{(i)}}$ is a difference of Zariski-open sets.

(c) Each $U_{e}^{\sim}$ is $\operatorname{Ad}^{*}(G)$-invariant.

Given $e \in \mathscr{E}$, let $S(e)=\left\{j: e_{j}=e_{j-1}+1\right\}, T(e)=\left\{j: e_{j}=e_{j-1}\right\}$, letting $e_{0}=0$ by convention, and let

$$
V_{S(e)}=\mathbf{R}-\operatorname{span}\left\{l_{j}: j \in S(e)\right\}, \quad V_{T(e)}=\mathbf{R}-\operatorname{span}\left\{l_{j}: j \in T(e)\right\} .
$$

Then

(d) The set $\Sigma_{e}^{\sim}=U_{e}^{\sim} \cap V_{T(e)}$ is nonempty and Zariski-open in the Zariski-closed set obtained by intersecting $V_{T(e)}$ with the complement of $\bigcup\left\{U_{e^{\prime}}^{-}: e^{\prime}>e\right\}$.

(e) $\operatorname{Ad}^{*}(G) \Sigma_{e}^{-}=U_{e}^{\sim}$.

(f) The elements of $\Sigma_{\tilde{e}}^{\sim}$ form a cross-section for the $\operatorname{Ad}^{*}(G)$-orbits in $U_{e}^{\sim}$. 
(g) There is a rational, nonsingular map $P_{e}: \Sigma_{e}^{-} \times V_{S(e)} \rightarrow U_{e}^{\sim}$ such that for each $l \in \Sigma_{e}^{-}, P_{e}(l, \cdot)$ is a polynomial map whose graph is the orbit $\mathrm{Ad}^{*}(G) l$. If $l \in U_{e}^{-}$, then $P_{e}^{-1}(l)=\left(l^{\prime}, l^{\prime \prime}\right)$ where $l^{\prime \prime}$ is the projection of $l$ onto $V_{S(e)}$ along $V_{T(e)}$, and $l^{\prime}$ is the unique point in $\operatorname{Ad}^{*}(G) l \cap V_{T(e)}$.

Thus $\cup_{e \in \mathscr{E}} \Sigma_{e}^{-}$is a cross-section for all the $\mathrm{Ad}^{*}(G)$-orbits in $\mathrm{g}^{*}$.

Proof. Parts (a)-(b) are proved essentially as in Proposition 2. Part(c) is an easy consequence of the fact that the $g_{j}$ are ideals. For $e=e^{(1)},(\mathrm{d})-(\mathrm{g})$ are proved in $[\mathbf{1 0}$, pp. 525-527], (see also [3]), and it is not hard to adapt that proof to the general case.

3. We now give a first description of $\rho=\operatorname{Ind}_{K \rightarrow G}(\chi)$ as a direct integral of irreducibles. (Recall that $\chi$ is 1-dimensional and corresponds to $f \in \mathfrak{H}^{*}$.) Let $\mathfrak{f}=\mathfrak{g}_{0} \subsetneq \mathfrak{g}_{1} \varsubsetneqq \cdots \subsetneq \mathfrak{g}_{m}=\mathfrak{g}$ be a chain of subalgebras of the sort considered in Proposition 2, and let $G_{j}=\exp \left(\mathrm{g}_{j}\right)$. Choose elements $Y_{j} \in \mathfrak{g}_{j} \backslash \mathfrak{g}_{j-1}(1 \leqslant j \leqslant m)$ and let $F=\mathbf{R}-\operatorname{span}\left\{Y_{1}, \ldots, Y_{m}\right\}$; let $l_{1}, \ldots, l_{m}$ be the basis of $\mathfrak{f}^{\perp} \subseteq \mathrm{g} *$ "dual" to $Y_{1}, \ldots, Y_{m}$ (so that $l_{i}\left(Y_{j}\right)=\delta_{i j}$ ). Note that $F^{\perp}=\mathfrak{f}^{*}$ in a natural way. Define an extension $f^{\prime} \in \mathrm{g}^{*}$ by $f^{\prime} \mid \mathfrak{f}=f, f^{\prime} \in F^{\perp}$.

Let $i$ be the smallest integer such that $U_{d^{(1)}} \cap\left(f^{\prime}+\mathfrak{H}^{\perp}\right) \neq \varnothing$, where $U_{d^{(i)}}$ is defined as in $\S 2$, and write $d=d^{(i)} ; V_{0}=U_{d} \cap\left(f^{\prime}+\mathfrak{H}^{\perp}\right)$ is a Zariski-open set in $f^{\prime}+\mathfrak{f}^{\perp}$. Define

$$
R_{1}=\left\{j: d_{j}=d_{j-1}\right\}, \quad R_{2}=\left\{j: d_{j}=2+d_{j-1}\right\} .
$$

From the note after Proposition 2, $R_{1}$ and $R_{2}$ are complementary subsets of $\{1,2, \ldots, m\}$. Let $E_{i}=\mathbf{R}-\operatorname{span}\left\{l_{j}: j \in R_{i}\right\}$ and let $r_{i}=\operatorname{card}\left(R_{i}\right), i=1,2$.

As we will see, the irreducibles appearing in $\rho$ are those whose $\operatorname{Ad}^{*}(G)$-orbits $\mathcal{O}_{1}$ meet $f^{\prime}+\mathfrak{f}^{\perp}$. Our first step is to show that, generically, these are precisely the orbits meeting the lower dimensional space $f^{\prime}+E_{1}$; this yields a direct integral decomposition of $\rho$ over $f^{\prime}+E_{1}$ (Theorem 2 below). Theorem 2 simplifies the problem of determining multiplicities, but further refinements are needed before we arrive at a solution. The following technical result is our main tool: it shows that orbits in $U_{d}$ that meet $f^{\prime}+f^{\perp}$ must also meet $f^{\prime}+E_{1}$ (thereby allowing us to prove Theorem 2), and it is also essential in later refinements. It may help to note that the sets $U_{d}$ are not necessarily $\operatorname{Ad}^{*}(G)$-invariant, unlike the $U_{e}^{\sim}$ of Theorem 1; this makes part (b) of the corollary below particularly important.

If $d \in \mathscr{D}, l \in U_{d}$, and $R_{2}(d)=\left\{i_{1}<\cdots<i_{k}\right\}$ we define an "action basis at $l \in U_{d}$ " to be any set of vectors $\mathscr{Y}=\left\{Y_{1}, \ldots, Y_{k}\right\}$ such that

$$
\operatorname{ad}^{*}\left(Y_{i}\right) P_{j_{i}}(l)=P_{j_{i}}\left(l_{j_{l}}\right) \text { and } \quad Y_{i} \in \mathrm{g}_{j_{l}} \quad(1 \leqslant i \leqslant k) \text {. }
$$

Given an action basis at $l \in U_{d}$, we define a map $\psi_{l}: \mathbf{R}^{k} \rightarrow \mathfrak{g}^{*}$ via

$$
\psi_{l}(t)=\operatorname{Ad}^{*}\left(\exp \left(t_{1} Y_{1}\right) \cdots \exp \left(t_{k} Y_{k}\right)\right) l
$$

and denote its range by $M_{l}$. 
Proposition 3. Given a chain of subalgebras $\mathfrak{f}=\mathfrak{g}_{0} \subseteq \cdots \subseteq \mathfrak{g}_{m}=\mathfrak{g}$, fix vectors $X_{i} \in \mathfrak{g}_{i} \backslash \mathfrak{g}_{i-1}$ and define dimension indices $\mathscr{D}$ as above. Fix $d \in \mathscr{D}$ and let $\mathfrak{H}^{\perp}=E_{1}$ $\oplus E_{2}$ be the corresponding splitting. If $\mathscr{Y}=\left\{Y_{1}, \ldots, Y_{k}\right\}$ is any action basis at $l \in U_{d}$, the variety $M_{l}$ has the following properties:

(a) $M_{l} \subseteq U_{d} \cap\left(l+\mathfrak{H}^{\perp}\right)$.

(b) If $l_{1} \in M_{l}$ and $\mathscr{J}^{\prime}=\left\{Y_{1}^{\prime}, \ldots, Y_{k}^{\prime}\right\}$ is any action basis at $l_{1}$, then the corresponding variety $M_{l_{1}}=M_{l_{1}}\left(\mathscr{Y}^{\prime}\right)$ satisfies $M_{l}=M_{l_{1}}$.

(c) If $\operatorname{Pr}_{2}$ is the projection of $\mathrm{g}^{*}=\mathfrak{f}^{*} \oplus E_{1} \oplus E_{2}$ onto $E_{2}$, the map $t \rightarrow \operatorname{Pr}_{2} \psi_{l}(t)$ is a diffeomorphism from $\mathbf{R}^{r_{2}}$ onto $E_{2}$.

Furthermore, $U_{d}$ may be covered by a finite number of Zariski-open sets $Z_{\alpha} \subseteq \mathrm{g}^{*}$ on which are defined rational, nonsingular maps $Y_{i}: Z_{\alpha} \rightarrow \mathfrak{g}_{j_{i}}\left(1 \leqslant i \leqslant k=r_{2}\right)$ such that $\left\{Y_{1}(l), \ldots, Y_{k}(l)\right\}$ is an action basis at l for each $l \in Z_{\alpha} \cap U_{d}$.

COROLlaRy. Given a chain of subalgebras from $\mathfrak{f}$ to $\mathfrak{g}$, fix an $l^{\prime} \in \mathfrak{g}$ *and consider all indices $\mathscr{D}_{0} \subseteq \mathscr{D}$ such that $U_{d}$ meets $l^{\prime}+\mathfrak{H}^{\perp}$. If $d \in \mathscr{D}_{0}$, then for each $l \in U_{d} \cap\left(l^{\prime}\right.$ $+\mathfrak{I}^{\perp}$ ) we may define a submanifold $M_{\text {l }}$ through l such that

(a) $M_{l} \subseteq l^{\prime}+\mathfrak{l}^{\perp}$.

(b) $M_{l} \subseteq U_{d}$ and $M_{l} \subseteq \mathcal{O}_{l}=\operatorname{Ad}^{*}(G) l$.

(c) The $M_{l}$ are "coherently defined": if $l_{1} \in M_{l}$, then $M_{l_{1}}=M_{l}$. Thus the $M_{l}$ do not depend on the base point, and partition $U_{d}$.

(d) For all $l \in U_{d} \cap\left(l^{\prime}+\mathfrak{l}^{\perp}\right), M_{l}$ meets $l^{\prime}+E_{1}$ in a unique point. (In particular, $U_{d} \cap\left(l^{\prime}+\mathfrak{H}^{\perp}\right)=\bigcup\left\{M_{l}: l \in U_{d} \cap\left(l^{\prime}+E_{1}\right)\right\}$ and if $d$ is the largest index in $\mathscr{D}$ such that $U_{d}$ meets $l^{\prime}+\mathfrak{H}^{\perp}$, then $U_{d}$ meets both $l^{\prime}+\mathfrak{l}^{\perp}$ and $l^{\prime}+E_{1}$ in nonempty Zariski-open sets.)

(e) For all $l \in U_{d} \cap\left(l^{\prime}+\mathfrak{H}^{\perp}\right), \mathrm{Pr}_{2}: M_{l} \rightarrow E_{2}$ is an onto diffeomorphism.

Proof. If $l \in U_{d}$, define $M_{l}$ using any action basis at $l$, as in Proposition 3. Since $l \in l^{\prime}+\mathfrak{l}^{\perp} \Rightarrow l^{\prime}+\mathfrak{l}^{\perp}=l+\mathfrak{l}^{\perp}$, (a)-(c), (e) are immediate. Since $\operatorname{Pr}_{2}\left(M_{l}\right)=E_{2}$, there is a unique $l_{1} \in M_{l}$ such that $\operatorname{Pr}_{2}\left(l_{1}\right)=\operatorname{Pr}_{2}\left(l^{\prime}+E_{1}\right)=\operatorname{Pr}_{2}\left(l^{\prime}\right)$. Since $M_{l} \subseteq l^{\prime}$ $+\mathfrak{f}^{\perp}, l_{1}=l^{\prime}+l_{0}$ for some $l_{0} \in \mathfrak{H}^{\perp}$. But $\operatorname{Pr}_{2}\left(l_{1}\right)=\operatorname{Pr}_{2}\left(l^{\prime}\right)+\operatorname{Pr}_{2}\left(l_{0}\right)$, so $\operatorname{Pr}_{2}\left(l_{0}\right)=$ 0 ; thus, $l_{0} \in \mathfrak{H}^{\perp} \cap \operatorname{ker} \operatorname{Pr}_{2}=E_{1}$ and we have $l_{1} \in l^{\prime}+E_{1}$. This proves (d). The parenthetical remarks in (d) follow from the way $\mathscr{D}$ is ordered, as in Proposition 2.

Note. If $d \in \mathscr{D}$, Proposition 3 shows that we may partition $U_{d}$ into varieties $M_{l}$, each of which maps diffeomorphically onto $E_{2}$ under $\operatorname{Pr}_{2}$. In a natural sense the $M_{1}$ vary rationally with $l$. Intersections of cosets of $\mathfrak{f}^{\perp}$ with $U_{d}$ also partition $U_{d}$, but this partition is coarser than that determined by the $M_{l}$. The transversality property (d) of the Corollary will be important in studying direct integral decompositions. Though the definition of the $M_{l}$ is rather ad hoc, the coherence property suggests that they are natural objects, and they are very useful; we do not know a canonical description of them.

Proof of Proposition 3. We work by induction on $\operatorname{dim}(g / \mathfrak{l})$, the result being trivial when this is zero. So assume everything is true for $K$ and $G_{m-1}$. We denote objects associated with $G_{m-1}$ by tildes. Using the same chain of subalgebras in $\mathfrak{g}_{m-1}$ as in $\mathfrak{g}$, we single out the indices $\mathscr{D}^{\sim}$ and layers $U_{d}^{\sim}$ partitioning $\mathfrak{g}_{m-1}^{*}$. The map 
$J\left(d_{0}, \ldots, d_{m}\right)=\left(d_{0}, \ldots, d_{m-1}\right)$ carries $\mathscr{D}$ onto $\mathscr{D}^{\sim}$. In fact, it is at most $2: 1$, and $J$

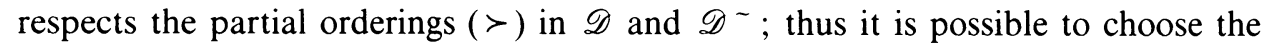
finer linear orderings $(<)$ so that $d_{1}<d_{2} \Rightarrow J\left(d_{1}\right) \leqslant J\left(d_{2}\right)$. Let $d$ be a fixed index in $\mathscr{D}$.

Clearly $P_{m-1}\left(U_{d}\right) \subseteq U_{J(d)}$. Notice that $R_{i}^{-}=R_{i} \cap\{0,1, \ldots, m-1\}$ where $R_{i}^{\sim}$ are the indices for $J(d)$. Also, $U_{d}$ is a union of $\mathbf{R} l_{m}$-cosets: if $l \in U_{d}$ and $l^{\prime}=l+c l_{m}$, we have $\operatorname{Ad}^{*}(G)$-orbits $\mathcal{O}_{l}, \mathcal{O}_{l^{\prime}}$ of equal dimension since $\operatorname{ad}^{*}(\mathrm{~g}) l_{m}=0$. Because $l^{\prime}\left|\mathfrak{g}_{m-1}=l\right| \mathfrak{g}_{m-1}$, we must have $d(l)=d\left(l^{\prime}\right)=d$.

There are two cases to consider.

Case 1. $m \in R_{1}$. Then $d_{m}=d_{m-1}$, and if $l \in U_{d}$ its $G$-orbit is mapped diffeomorphically to the $G_{m-1}$ orbit of $l^{-}=P_{m-1}(l)$.

Let us first consider the construction of rationally varying action bases on $U_{d}$. We have noted that $P_{m-1}\left(U_{d}\right) \subseteq U_{J(d)}$. Define $Q: \mathrm{g}_{m-1}^{*} \rightarrow \mathrm{g}^{*}$ by $Q l\left(X_{m}\right)=0, P_{m-1} \circ Q$ $=i d$. Suppose we have covered $U_{J(d)}^{\sim}$ by Zariski-open sets $Z_{\alpha}^{\sim} \subseteq \mathfrak{g}_{m-1}^{*}$ on which rational maps $\left\{Y_{i}^{\sim}(l)\right\}$ are defined. We then construct the maps $\psi_{\alpha}^{\sim}\left(l^{\sim}, t\right)$ and manifolds $M_{l^{-}}^{\alpha}$ for $l^{\sim} \in Z_{\alpha}^{\sim} \cap U_{j(\tilde{d})}$. Using the same set of indices $\alpha$, let $Z_{\alpha}=$ $P_{m-1}^{-1}\left(Z_{\alpha}^{\sim}\right)$ and define $Y_{i}(l)=Y_{i}^{\sim}\left(l^{\sim}\right)$ for $1 \leqslant i \leqslant r_{2}$, where $l^{\sim}=P_{m-1}(l)$. Here, $r_{2}^{\sim}=r_{2}, E_{2}^{\sim}=E_{2}, E_{1}=E_{1}^{\sim} \oplus \mathbf{R} l_{m}, \operatorname{Pr}_{2}=\operatorname{Pr}_{2}^{\sim} \circ P_{m-1}$. Since $P_{j}^{\sim} \circ P_{m-1}=P_{j}$ for $j<m$, and since the actions of $G_{j}$ on $\mathrm{g}^{*}$ and $\mathrm{g}_{j}^{*}$ commute with $P_{j}$, it is clear that the $Y_{i}(l)$ on $Z_{\alpha}$ give an action basis at each $l \in U_{d} \cap Z_{\alpha}$.

Now let $l \in U_{d}$ and $\mathscr{Y}=\left\{Y_{1}, \ldots, Y_{k}\right\}$ be an action basis at $l$; define $\psi_{l}(t)$ as in (3). Since $m \notin R_{2}(d), \mathscr{Y}$ is also an action basis at $l^{\sim}=P_{m-1}(l)$ in $U_{J(d)}$. Since $l=Q l^{-}+c l_{m}$ for some $c \in \mathbf{R}$, we see that $P_{m-1} \psi_{l}(t)=\psi_{l^{-}}(t)$; hence $P_{m-1}\left(M_{l}\right)=$ $M_{1}$. . Therefore, by induction,

$$
M_{l} \subseteq P_{m-1}^{-1}\left(M_{l^{-}}\right) \subseteq P_{m-1}^{-1}\left(l^{\sim}+\mathfrak{H}^{\perp, \sim}\right)=l+\mathfrak{t} \perp
$$

and the map $t \rightarrow \operatorname{Pr}_{2} \psi_{l}(t)=\operatorname{Pr}_{2}^{-} \psi_{l^{-}}(t)$ is a diffeomorphism onto $E_{2}$. Also, $l^{\sim} \in$ $P_{m-1}\left(U_{d}\right) \subseteq U_{J(\tilde{d})}$ and $P_{m-1}\left(M_{l}\right)=M_{1-\subseteq} \subseteq U_{J(d)} \cap \mathcal{O}_{1^{-}}$where $\mathcal{O}_{1^{-}}=\operatorname{Ad}^{*}\left(G_{m-1}\right) l^{\sim}$ $=P_{m-1}\left(\operatorname{Ad}^{*}(G) l\right)$. Thus, $l^{\prime} \in M_{l} \Rightarrow l^{\prime} \in \mathcal{O}_{l} \Rightarrow P_{m-1}\left(l^{\prime}\right) \in \mathcal{O}_{1^{-}} \Rightarrow \operatorname{dim} \operatorname{Ad}^{*}(G) l^{\prime}=$ $\operatorname{dim} \operatorname{Ad}^{*}\left(G_{m-1}\right) P_{m-1}\left(l^{\prime}\right)$. Since $P_{m-1}\left(l^{\prime}\right) \in U_{J(d)}$ and $d_{m}=d_{m-1}$ in $d$, we conclude that $l^{\prime} \in U_{d}$ and hence that $M_{l} \subseteq U_{d}$.

For (b), let $l_{1} \in M_{l}$, let $\mathscr{Y}_{1}=\left\{Y_{1}^{\prime}, \ldots, Y_{k}^{\prime}\right\}$ be any action basis at $l_{1}$, and let $M_{l_{1}}=M_{l_{1}}\left(\mathscr{Y}_{1}\right)$. We have just seen that $P_{m-1}\left(M_{l}\right)=M_{l}$ - and we know that $U_{d}$ is saturated in the $\mathbf{R} l_{m}$ direction; thus $l_{1}^{\sim}=P_{m-1}\left(l_{1}\right) \in M_{l^{-}}=M_{l^{-}}(\mathscr{Y})$. Now $\mathscr{Y}_{1}$ is also an action basis at $l_{1}^{\sim} \in U_{J(d)}^{\tilde{d})}$, and by induction we get $M_{l^{-}}=M_{l_{1}^{-}}$. But $M_{l}$ and $M_{l_{1}}$ lie in the same $\operatorname{Ad}^{*}(G)$-orbit, which is mapped bijectively onto the $\operatorname{Ad}^{*}\left(G_{m-1}\right)$ orbit of $l^{-}$, and the latter contains $M_{l^{-}}, M_{l_{1^{-}}}$. Thus, $M_{l}(\mathscr{Y})=M_{l_{1}}\left(\mathscr{Y}_{1}\right)$ and (a)-(c) are proved in this case.

Case 2. $m \in R_{2}(D)$. Now $d_{m}=2+d_{m-1}$ and $U_{d}$ consists of points whose $\operatorname{Ad}^{*}(G)$-orbit is saturated with respect to $P_{m-1}$.

Let $l \in U_{d}, l^{-}=P_{m-1}(l) \in P_{m-1}\left(U_{d}\right) \subseteq U_{J(d)}$. The $\operatorname{Ad}^{*}(G)$-orbit $\mathcal{O}_{l}$ is a union of $\mathbf{R} l_{m}$-cosets. Let $U_{d}=A \backslash B$ where $A, B$ are Zariski-open sets in $\mathrm{g}^{*}$ (see Proposition 2). For any $l \in U_{d}, \operatorname{dim} \mathcal{O}_{1}=r=2 r_{2}+d_{0}$, where $d_{0}=$ $\operatorname{dim} \operatorname{Ad}^{*}(K)(l \mid \mathfrak{f})$. Thus, the maps $A_{l}(X)=\operatorname{ad}^{*}(X) l, A_{l}: \mathrm{g} \rightarrow \mathrm{g}^{*}$ for $l \in \mathrm{g}^{*}$, have rank $r$ for all $l \in U_{d}$. Taking any convenient bases in $\mathrm{g}, \mathrm{g}^{*}$, we regard $A_{l}$ as a 
matrix; let $A_{l}^{\zeta}$ indicate the various $r \times r$ submatrices of $A_{l}$, and let $Z_{\zeta}=\{l \in$ $\mathrm{g}^{*}$ : det $\left.A_{l}^{\zeta} \neq 0\right\}$. These are Zariski-open sets in $\mathrm{g}^{*}$ that cover $U_{d}=A \backslash B$. (Some may be empty, or disjoint from $U_{d}$; eliminate those.) If $l \in U_{d} \cap Z_{\zeta}$, then by definition of $U_{d}$, and the Case 2 property, $l_{m} \in \operatorname{range}\left(A_{l}\right)$. The submatrix inverse $\left(A_{l}^{\zeta}\right)^{-1}$ is rational and nonsingular on $Z_{\zeta}$. These observations allow us to form rational, nonsingular functions $X(l) \in \mathrm{g}$ on $Z_{\zeta}$, such that $\mathrm{ad}^{*}(X(l)) l=l_{m}$ for all $l \in Z_{\xi} \cap U_{d}$. [Detail: specifying an $r \times r$ submatrix $A_{l}^{\zeta}$ of the $n \times n$ matrix $A_{l}$ amounts to defining projections $P$ in $\mathrm{g}, Q$ in $\mathrm{g}^{*}$ onto subspaces $E_{P} \subseteq \mathrm{g}, E_{Q} \subseteq \mathrm{g}^{*}$, of dimension $r$ such that $A_{l}^{\zeta}$ identifies with $Q A_{l} P: E_{P} \rightarrow E_{Q}$. Now $\operatorname{dim}\left(\operatorname{range} A_{l}\right)=$ $\operatorname{dim}^{*}(\mathrm{~g}) l=r$ for all $l \in U_{d}$. Given $A_{l}^{\zeta}$ and $Z_{\zeta}$ as above, we have

(i) $\operatorname{dim} Q A_{l} P(\mathrm{~g})=r=\operatorname{dim}\left(\right.$ range $\left.A_{l}\right)$, hence

(ii) $Q$ : range $\left(A_{l}\right) \rightarrow E_{Q}$ is an isomorphism for all $l \in Z_{\xi}$.

Let $T_{l}^{\zeta}=Q A_{l} P$ for $l \in Z_{\zeta}$. Then $\left(T_{l}^{\zeta}\right)^{-1}$ is rational, nonsingular in $l$, and the map $X(l)=\left(T_{l}^{\zeta}\right)^{-1} Q\left(l_{m}\right)$ is rational, with

$$
Q A_{l}(X(l))=Q\left(l_{m}\right) \quad \text { all } l \in Z_{\zeta} .
$$

For $l \in U_{d} \cap Z_{\xi}$ we have $l_{m} \in \operatorname{range}\left(A_{l}\right)$, hence by (ii) we must have $A_{l}(X(l))=$ $\operatorname{ad}^{*}(X(l)) l=l_{m}$.]

We know that $\operatorname{ad}^{*}(\mathrm{~g}) l_{m}=0$; thus if $l \in U_{d} \cap Z_{\zeta}$ and $x \in G_{m-1}$, we have

$$
\begin{aligned}
& \operatorname{Ad}^{*}\left(x \cdot \exp \left(t_{r} X(l)\right)\right) l=\operatorname{Ad}^{*}(x) l+t_{r} l_{m}, \\
& \operatorname{Ad}^{*}(x \cdot \exp (\mathbf{R} X(l))) l=P_{m-1}^{-1}\left(\operatorname{Ad}^{*}(x) P_{m-1}(l)\right) .
\end{aligned}
$$

Now let $\left\{Y_{i}(l): 1 \leqslant i \leqslant r_{2}-1\right\}$ be the inductively defined rational maps on Zariski-open sets $Z_{\alpha}^{\sim} \subseteq \mathfrak{g}_{m-1}^{*}$, covering $U_{J(d)}$. Let $Z_{\alpha}=P_{m-1}^{-1}\left(Z_{\alpha}^{\sim}\right)$ and consider the family of Zariski-open sets $Z_{\zeta, \alpha}=Z_{\zeta} \cap Z_{\alpha} \subseteq \mathrm{g}^{*}$, which cover $U_{d}$. In Case 2, $r_{2}^{\sim}=r_{2}-1, E_{1}=E_{1}^{-}, E_{2}=E_{2}^{\sim} \oplus \mathbf{R} l_{m}$. On $Z_{\zeta, \alpha}$ define $Y_{r_{2}}(l)=X(l)$ as above, and $Y_{i}(l)=Y_{i}^{\sim}\left(P_{m-1}(l)\right)$ for $1 \leqslant i \leqslant r_{2}-1$. Again, it is obvious that $\left\{Y_{i}(l)\right\}$ is an action basis at each $l \in U_{d} \cap Z_{\zeta, \alpha}$.

Next consider a fixed $l \in U_{d}$, action basis $\mathscr{Y}=\left\{Y_{1}, \ldots, Y_{r_{2}}\right\}$ at $l$, and manifold $M_{l}=\psi_{l}\left(\mathbf{R}^{r_{2}}\right)$. Then $\mathscr{Y} \sim=\left\{Y_{1}, \ldots, Y_{r_{2}-1}\right\}$ is an action basis at $l \sim=P_{m-1}(l) \in U_{J(d)}$ and if $M_{l^{-}}=M_{l^{-}}\left(\mathscr{Y}^{\sim}\right)$, we have

$$
M_{l}=P_{m-1}^{-1}\left(M_{l^{-}}\right)
$$

by the same argument as in (4), taking $X(l)=Y_{r_{2}}$. From this we also see that, if $l=Q l^{\sim}+c l_{m}(c \in \mathbf{R})$, then

$$
\psi_{l}(t)=\left(\psi_{l}-\left(t^{\prime}\right)+t_{r_{2}} l_{m}\right)+c l_{m}
$$

where $t=\left(t^{\prime}, t_{r_{2}}\right)$. This shows that $M_{l} \subseteq l+\mathfrak{l}^{\perp}$, and that $r \rightarrow \operatorname{Pr}_{2} \psi_{l}(t)$ is a diffeomorphism. To show that $M_{l} \subseteq U_{d}$ we note that

$$
P_{m-1}\left(M_{l}\right) \subseteq M_{l} \cap \cap \mathcal{O}_{1} \cap \cap \tilde{U_{J(d)}} \text { and } M_{l} \subseteq \mathcal{O}_{l} \text {. }
$$

Thus if $l^{\prime} \in M_{l}$, we have $\operatorname{dim} \operatorname{Ad}^{*}(G) l^{\prime}=\operatorname{dim} \mathcal{O}_{1}=2+\operatorname{dim} \mathcal{O}_{1^{-}}=2+$ $\operatorname{dim} \operatorname{Ad}^{*}\left(G_{m-1}\right) P_{m-1}\left(l^{\prime}\right)$. Since $P_{m-1}\left(l^{\prime}\right) \in U_{J(d)}^{\tilde{(})}$ and $d_{m}=2+d_{m-1}$, we conclude that $l^{\prime} \in U_{d}$ and $M_{l} \subseteq U_{d}$. 
Finally, let $l_{1} \in M_{l}$ and let $\mathscr{Y}_{1}=\left\{Y_{1}^{\prime}, \ldots, Y_{r_{2}}^{\prime}\right\}$ be any action basis at $l_{1}$. Then $\mathscr{Y}_{1}^{\sim}=\left\{Y_{1}^{\prime}, \ldots, Y_{r_{2}-1}^{\prime}\right\}$ is an action basis at $l_{1}^{\sim}=P_{m-1}\left(l_{1}\right) \in U_{J(d)}$. Since $M_{l}$ is $\mathbf{R} l_{m}$-saturated, $l_{1}^{\sim} \in M_{1} \sim$. Hence, by induction, $M_{l_{1}}\left(\mathscr{Y}_{1} \sim\right)=M_{l}-(\mathscr{Y} \sim)$. But by (5) we get $M_{l_{1}}=M_{l}$, which proves (c).

THEOREM 2. Let $\chi \in K^{\wedge}$ be a one-dimensional representation, specify a chain of subalgebras $\mathfrak{f}=\mathfrak{g}_{0} \subseteq \cdots \subseteq \mathfrak{g}_{m}=\mathfrak{g}$, and let $d$ be the largest index in $D$ such that $U_{d}$ meets $f^{\prime}+\mathfrak{f}^{\perp}$. Then $U_{d} \cap\left(f^{\prime}+\mathfrak{f}^{\perp}\right)$ is Zariski-open in $f^{\prime}+\mathfrak{f}$ and $U_{d} \cap\left(f^{\prime}+E_{1}\right)$ is a nonempty Zariski-open set in $f^{\prime}+E_{1}$. If $d l$ is Euclidean measure on $E_{1}$, then

$$
\operatorname{Ind}_{K \rightarrow G}(\chi) \cong \int_{E_{1}}^{\oplus} \pi_{l+f^{\prime}} d l
$$

Proof. Again, we work by induction on $\operatorname{dim}(\mathfrak{g} / \mathfrak{f})$. The assertion about $U_{d} \cap\left(f^{\prime}\right.$ $+E_{1}$ ) has been proved in Proposition 3. We assume everything true for $K$ and $G_{m-1}$ and adopt the notation of the proposition, denoting objects in $G_{m-1}$ by tildes. If $d^{\sim}=J(d)=\left(d_{0}, \ldots, d_{m-1}\right)$ then $U_{d}$ is $P_{m-1}$-saturated and the set $U_{d} \cap\left(f^{\prime}+\mathfrak{f}^{\perp}\right)$ is Zariski-open in $f^{\prime}+\mathfrak{H}^{\perp}$, so its $P_{m-1}$-image is a dense open set in $\left(f^{\sim}+\mathfrak{f}^{\perp}\right) \cap$ $U_{J(d)}$. Hence $U_{d}$ is the first layer in $\mathfrak{g}_{m-1}^{*}$ to meet $f^{\sim}+\mathfrak{l}^{\perp, \sim}$. We let

$$
V_{f}=\left(f^{\prime}+\mathfrak{f}^{\perp}\right), \quad V_{0}=U_{d} \cap\left(f^{\prime}+\mathfrak{f}^{\perp}\right)
$$

with $V_{f}^{\sim}, V_{0}^{\sim}$ the corresponding sets in $f^{\sim}+\mathcal{\perp} \sim$. Since $U_{d}$ is $\mathbf{R} l_{m}$-saturated, $P_{m-1}\left(U_{d}\right) \subseteq U_{d^{-}}$, and $P_{m-1}\left(V_{f}\right)=V_{f}^{\sim}$, we see that $P_{m-1}\left(V_{0}\right) \subseteq V_{0}^{\sim}$ and both are Zariski-open sets in $V_{f}^{\sim}$.

By the inductive hypothesis we have

$$
\begin{aligned}
\rho & \cong \operatorname{Ind}_{G_{m-1} \rightarrow G}\left(\operatorname{Ind}_{K \rightarrow G_{m-1}}(\chi)\right) \\
& \cong \operatorname{Ind}_{G_{m-1} \rightarrow G}\left(\int_{E_{1^{-}}}^{\oplus} \sigma_{l^{-}+f^{-}} d l^{-}\right) \\
& \cong \int_{f^{-}+E_{1}^{-}}^{\oplus} \operatorname{Ind}_{G_{m-1} \rightarrow G^{-}}\left(\sigma_{l^{-}}\right) d l^{-}
\end{aligned}
$$

where $\sigma_{l-} \in G_{m-1}^{\wedge}$ corresponds to $l^{\sim}$.There are two cases to consider. In either case $P_{m-1}\left(E_{1}\right)=E_{1}^{\sim}$, so $P_{m-1}\left(V_{0}\right) \cap\left(f^{\sim}+E_{1}^{\sim}\right)=P_{m-1}\left(V_{0}\right) \cap P_{m-1}\left(f^{\prime}+E_{1}\right) \supseteq$ $P_{m-1}\left(U_{d} \cap\left(f^{\prime}+E_{1}\right)\right)$, which is an open dense semialgebraic subset of $f^{\sim}+E_{1}^{\sim}$. Thus $P_{m-1}\left(V_{0}\right)$ meets $f^{\sim}+E_{1}^{\sim}$ in a set having full Euclidean measure.

Case 1. $m \in R_{1}$. Then $P_{m-1}$ is a diffeomorphism mapping each $G$-orbit $\mathcal{O}_{l}$, $l \in U_{d}$, to a $G_{m-1}$-orbit $\mathcal{O}_{1-}\left(l^{\sim}=P_{m-1}(l)\right)$. Define $Q: \mathrm{g}_{m-1}^{*} \rightarrow \mathrm{g}^{*}$ by $Q l^{\sim}\left(Y_{m}\right)=0$, $P_{m-1} Q=i d$. In this case $E_{1}=E_{1}^{\sim} \oplus \mathbf{R} l_{m}, E_{2}=E_{2}^{\sim}, \operatorname{Pr}_{2}=\operatorname{Pr}_{2}^{\sim} P_{m-1}$, and if we let $\operatorname{Ad}^{*}(x)$ act in $\mathfrak{g}_{m-1}^{*}$ by restriction to this invariant subspace then $\operatorname{Ad}^{*}(x)$ commutes with $P_{m-1}$ for all $x \in G$. From (d) of Proposition 1 we have, for all $l^{\sim} \in P_{m-1}\left(V_{0}\right)$,

$$
\operatorname{Ind}_{G_{m-1} \rightarrow G}\left(\sigma_{l^{-}}\right) \cong \int_{\mathbf{R}}^{\oplus} \pi_{l+\alpha l_{m}} d \alpha
$$


where $l$ is any extension of $l^{\sim}$. Taking $l=Q l^{\sim}$, the integral (7) may be rewritten as

$$
\begin{aligned}
\rho & \cong \int_{\left(f^{-}+E_{1}^{\sim}\right) \cap P_{m-1}\left(V_{0}\right)}^{\oplus} \operatorname{Ind}_{G_{m-1} \rightarrow G}\left(\sigma_{l^{-}}\right) d l^{\sim} \\
& \cong \int_{\left(f^{-}+E_{1}^{\sim}\right) \cap P_{m-1}\left(V_{0}\right)}^{\oplus} \int_{\mathbf{R}}^{\oplus} \pi_{Q l^{-}+\alpha l_{m}} d \alpha d l^{\sim} \\
& \cong \int_{\left(f^{\prime}+E_{1}\right) \cap V_{0}}^{\oplus} \pi_{l} d l=\int_{E_{1}}^{\oplus} \pi_{f^{\prime}+l} d l,
\end{aligned}
$$

since $Q E_{1}^{\sim}+\mathbf{R} l_{m}=E_{1}$ and $V_{0}=P_{m-1}^{-1}\left(P_{m-1}\left(V_{0}\right)\right)\left(V_{0}\right.$ is $l_{m}$-saturated since $U_{d}$ is $)$.

Case 2. $m \in R_{2}$. Now $d_{m}=2+d_{m-1}$, and $U_{d}$ consists of points $l$ whose $G$-orbit $\mathcal{O}_{1}$ is saturated with respect to $l_{m}$, and $E_{1}=E_{1}^{\sim}$. Again, $P_{m-1}\left(V_{0}\right)$ has full

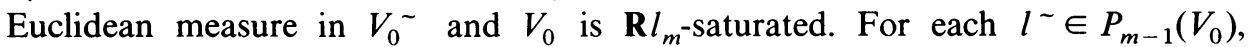
$Q l^{\sim} \in V_{0}$ and $\operatorname{Ind}_{G_{m-1} \rightarrow G}\left(\sigma_{l^{-}}\right)=\pi_{Q l^{-}}$. Since $Q\left(f^{\sim}+E_{1}^{\sim}\right)=f^{\prime}+E_{1}$, equation (7) yields

$$
\begin{aligned}
\rho & \cong \int_{f^{-}+E_{1}^{-}}^{\oplus} \pi_{Q l^{-}} d l^{\sim} \cong \int_{\left(f^{-}+E_{1}^{-}\right) \cap P_{m-1}\left(V_{0}\right)}^{\oplus} \pi_{Q l^{-}} d l^{\sim} \\
& \cong \int_{\left(f^{\prime}+E_{1}\right) \cap v_{0}}^{\oplus} \pi_{l} d l \cong \int_{E_{1}}^{\oplus} \pi_{f^{\prime}+l} d l
\end{aligned}
$$

which proves (6).

4. The weakness of Theorem 1 is that different elements in the domain of integration $f^{\prime}+E_{1}$ can correspond to the same element of $G^{\wedge}$. The subvarieties $M_{l}$ meet $f^{\prime}+E_{1}$ in a unique point, but intersections with $G$-orbits can be larger. Further cross-sectioning is needed to sort out the multiplicities. In this section we take care of the case of infinite generic multiplicity, and prepare the setting for the finite multiplicity case.

To unravel the direct integral decomposition of $\operatorname{Ind}_{K \rightarrow G}(\chi)$ we shall go back to the parametrization of $G$-orbits relative to a strong Mal'cev basis for $\mathfrak{g}$, given in Theorem 1, and the related cross-sections for families of orbits. These will be compared with the partial cross-sections used to obtain the direct integral decomposition over $f^{\prime}+E_{1}$ in $\S 3$.

Let $\mathfrak{l}^{\prime} \mathrm{g}_{0} \subseteq \mathrm{g}_{1} \subseteq \cdots \subseteq \mathrm{g}_{m}=\mathrm{g}$ be a chain of subalgebras. Pick basis vectors as in $\S 3$, define dimension indices $\mathscr{D}$ and layers $U_{d}$, and let $d \in \mathscr{D}$ be the largest index such that $U_{d}$ meets $f^{\prime}+\mathfrak{f}^{\perp}$. Defining the splitting $\mathfrak{f}^{\perp}=E_{1}(d) \oplus E_{2}(d)$ as in $\S 3$, we have shown that $U_{d}$ is also the first $\mathscr{D}$-layer to meet $f^{\prime}+E_{1}$, and that $U_{d} \cap\left(f^{\prime}+\mathfrak{f}^{\perp}\right)=\bigcup\left\{M_{l}: l \in U_{d} \cap\left(f^{\prime}+E_{1}\right)\right\}$. The intersection of $U_{d}$ with $f^{\prime}+E_{1}$ is Zariski-open in $f^{\prime}+E_{1}$.

Next, fix a strong Mal'cev basis in $\mathrm{g}$, define dimension indices $\mathscr{E}$ and layers $U_{e}^{-}$ partitioning $\mathrm{g}^{*}$ as in Theorem 1 . Let $e \in \mathscr{E}$ be the largest index such that $U_{e}^{-}$ meets $f^{\prime}+\mathfrak{H}^{\perp}$. Observe that

The index $e$ is also the largest index in $\mathscr{E}$ such that $U_{e}^{\sim}$ meets $f^{\prime}+E_{1}$ (and the intersection is Zariski-open). 
In fact, if $e^{\prime}>e$ in $\mathscr{E}$, it is clear that $U_{e^{\prime}}^{-} \cap\left(f^{\prime}+E_{1}\right)=\varnothing$. But $U_{e}^{\sim} \cap U_{d} \cap\left(f^{\prime}+\right.$ $\mathfrak{l}^{\perp}$ ) is Zariski-open in $f^{\prime}+\mathfrak{f}^{\perp}$. If $l$ is in this set, so is $M_{l}$ since $U_{e}^{\sim}$ is $G$-invariant. But then $M_{l}$ meets $f^{\prime}+E_{1}$ and so does $U_{e}^{\sim}$, as required.

For this index $e$, decompose $\mathrm{g}^{*}=V_{T(e)} \oplus V_{S(e)}$ as in Theorem 1; then $\Sigma_{e}^{-}=$ $V_{T(e)} \cap U_{e}^{\sim}$ is a semialgebraic cross-section for the orbits in $U_{e}^{\sim}$ and there is a birational nonsingular parametrizing map $P_{e}: \Sigma_{e}^{\sim} \times V_{S(e)} \rightarrow U_{e}^{\sim}$. If $\pi_{T}$ is the projection of $\mathrm{g}^{*}$ onto $V_{T(e)}$ along $V_{S(e)}$, define

$$
\phi=\pi_{T} \circ P_{e}^{-1} \mid U_{e}^{-} \cap\left(f^{\prime}+E_{1}\right) ;
$$

$\phi$ is a rational nonsingular map from this Zariski-open set in $f^{\prime}+E_{1}$ to orbit representatives in $\Sigma_{e}^{\sim}$. Let

$$
\begin{aligned}
& r_{1}=r_{1}(d)=\operatorname{dim} E_{1}, \quad r_{2}=r_{2}(d)=\operatorname{dim} E_{2}, \\
& k=\text { maximal (generic) rank of } d \phi, \text { for } l \in U_{e}^{-} \cap\left(f^{\prime}+E_{1}\right), \\
& k^{\prime}=r_{1}-k,
\end{aligned}
$$

and define

$$
E^{*}=\left(f^{\prime}+E_{1}\right) \cap U_{e}^{\sim} \cap U_{d} \cap\left\{l: \operatorname{rank} d \phi_{l}=k\right\} \text {. }
$$

This is a nonempty Zariski-open set in $f^{\prime}+E_{1}$. By a standard theorem, see [8, pp. 5-7], there is a foliation of $E^{*}$ with respect to $\phi$ and near each $l \in E^{*}$ we can find a centered rectangular coordinate neighborhood $N_{l}=I_{1} \times I_{2} \subseteq \mathbf{R}^{k} \times \mathbf{R}^{r_{1}-k}$ such that $\phi$ is a diffeomorphism on the transversal $I_{1} \times(0)$, constant on the "leaves" $(s) \times I_{2}, s \in I_{1}$, and has distinct values on each leaf. Each leaf in $N_{l}$, and in fact the whole $\phi$-leaf through $l$ in $E^{*}$, lies within a single orbit intersection $\operatorname{Ad}^{*}(G) l \cap\left(f^{\prime}\right.$ $\left.+E_{1}\right)$, with $G \cdot l \subseteq U_{e}^{-}$.

We now define a "defect index"

$$
\tau_{0}=\operatorname{dim} E_{1}-k=r_{1}(d)-\text { generic } \operatorname{rank}\left\{d \phi_{l}: l \in f^{\prime}+E_{1}\right\} .
$$

This will be given a canonical geometric interpretation in terms of orbit dimensions in the next section. We also define the following sets, which are semialgebraic since $P_{e}^{-1}$ is rational nonsingular

$$
\begin{aligned}
& \Sigma^{\chi}=\pi_{T} \circ P_{e}^{-1}\left(U_{e}^{\sim} \cap\left(f^{\prime}+\mathfrak{H}^{\perp}\right)\right), \\
& \Sigma^{1}=\pi_{T} \circ P_{e}^{-1}\left(U_{e}^{\sim} \cap\left(f^{\prime}+E_{1}\right)\right)=\phi\left(U_{e}^{\sim} \cap\left(f^{\prime}+E_{1}\right)\right), \\
& \Sigma^{*}=\pi_{T} \circ P_{e}^{-1}\left(E^{*}\right)=\phi\left(E^{*}\right) .
\end{aligned}
$$

Since $\phi$ has constant rank $k$ on $E^{*}$, we see that $\Sigma^{*}$ is covered by countably many submanifolds in $V_{T(e)}$ having dimension $k$, so that $\operatorname{dim} \Sigma^{*} \leqslant k$; the converse is obvious, so $\operatorname{dim} \Sigma^{*}=k$.

THEOREM 3. Let $\mathrm{g}$ be a nilpotent Lie algebra, $f$ a subalgebra, and $G, K$ the simply connected Lie groups. Let $\chi \in K^{\wedge}$ have $\operatorname{dim} \chi=1$, so $\chi=e^{2 \pi i f}$ with $f \in \mathfrak{f}^{*}$; if $P: \mathrm{g}^{*} \rightarrow \mathfrak{f}^{*}$ is the natural map, form $P^{-1}\left(\mathcal{O}_{\chi}\right)=f^{\prime}+\mathfrak{H}^{\perp}$, where $f^{\prime}$ is any extension of $f$ to $\mathfrak{g}$. Using a chain of subalgebras $\mathfrak{f}=\mathfrak{g}_{0} \subseteq \cdots \subseteq \mathfrak{g}_{m}=\mathfrak{g}$, define layers 
$\left\{U_{d}: d \in \mathscr{D}\right\} ;$ let $U_{d}$ be the first layer meeting $f^{\prime}+\mathfrak{H}^{\perp}$ and define the splitting $\mathfrak{H}^{\perp}=E_{1}(d) \oplus E_{2}(d)$ as above. Using a Mal'cev basis $X_{1}, \ldots, X_{n}$ for $\mathfrak{g}$, define layers $\left\{U_{e}^{\sim}: e \in \mathscr{E}\right\} ;$ let $U_{e}^{\sim}$ be the first layer that meets $f^{\prime}+\mathfrak{H}^{\perp}$ and define maps $P_{e}^{-1}$ and $\phi$ as above. Define $\Sigma^{x}, \Sigma^{1}, \Sigma^{*}$ as above. Then

(a) If $\phi^{\sim}=\pi_{T} \circ P_{e}^{-1} \mid U_{e}^{\sim} \cap\left(f^{\prime}+\mathfrak{f}^{\perp}\right)$, then $\operatorname{range}\left(\phi^{\sim}\right)=\Sigma^{x}$,

(11) generic $\operatorname{rank}\left\{d \phi_{l}: l \in f^{\prime}+\mathfrak{H}^{\perp}\right\}=$ generic $\operatorname{rank}\left\{d \phi_{l}: l \in f^{\prime}+E_{1}\right\}=k$

and $\operatorname{dim} \Sigma^{x}=k$.

(b) $\Sigma^{x}, \Sigma^{1}, \Sigma^{*}$ differ by sets having dimension less than $\operatorname{dim} \Sigma^{*}=k$, and so determine the same measure class $[\nu]$.

(c) If $\tau_{0}>0$ then

$$
\rho=\operatorname{Ind}_{K \rightarrow G}(\chi) \cong \int_{\Sigma^{x}}^{\oplus} \infty \cdot \pi_{l^{\prime}} d \nu\left(l^{\prime}\right) .
$$

Proof. The semialgebraic set $\Sigma^{*}$ has a stratification $\mathscr{P}=\left\{S_{1}, \ldots, S_{p}\right\}$ and $\operatorname{dim} \Sigma^{*}=k$. Let $\Sigma_{r}^{*}=$ union of the $k$-dimensional pieces and $\Sigma_{s}^{*}$ the union of the others. Let $\nu$ be the measure obtained by putting a nonvanishing $k$-form on each $k$-dimensional piece; clearly $\nu\left(\Sigma_{s}^{*}\right)=0$ and $\operatorname{dim} \Sigma_{s}^{*}<k$. Let us partition $E^{*}$ into the semialgebraic sets

$$
E_{r}^{*}=\phi^{-1}\left(\Sigma_{r}^{*}\right) \cap E^{*}, \quad E_{s}^{*}=\phi^{-1}\left(\Sigma_{s}^{*}\right) \cap E^{*} .
$$

Then $\Sigma_{r}^{*}$ is an open set in $\Sigma^{*}$, and since $\phi$ is continuous $E_{r}^{*}$ is open in $f^{\prime}+E_{1}$. To prove (a), and for later work, we will need information about the " $M_{l}$-saturant" $S_{A}=\bigcup\left\{M_{l}: l \in A\right\}$ of an open, dense, semialgebraic set in $U_{d} \cap\left(f^{\prime}+E_{1}\right)$, such as $A=E^{*}$. For the particular set $A_{0}=U_{d} \cap\left(f^{\prime}+E_{1}\right), S_{A_{0}}=U_{d} \cap\left(f^{\prime}+\mathfrak{H}^{\perp}\right)$ by Theorem 2, and has complement of lower dimension in $f^{\prime}+\perp^{\perp}$. More generally,

If $A$ is any dense, open, semialgebraic set in $U_{d} \cap\left(f^{\prime}+E_{1}\right)$, then $S_{A}=\bigcup\left\{M_{l}: l \in A\right\}$ is semialgebraic and contains an open, dense, subset of $f^{\prime}+\mathfrak{t}^{\perp}$, and its complement has dimension lower than $\operatorname{dim} \mathfrak{f}^{\perp}=r_{1}(d)+r_{2}(d)$.

To see this, cover $U_{d}$ with Zariski-open sets $V_{\alpha} \subseteq \mathrm{g}^{*}$ on which are defined rational nonsingular maps $\left\{X_{1}^{\alpha}(l), \ldots, X_{r_{2}}^{\alpha}(l)\right\}$ that give an action basis at each $l \in U_{d} \cap V_{\alpha}$; then $\psi_{\alpha}: V_{\alpha} \times \mathbf{R}^{r_{2}} \rightarrow \mathrm{g}^{*}$ defined as in (3) is rational nonsingular and $\psi_{\alpha}\left(l, \mathbf{R}^{r_{2}}\right)=M_{l}$ if $l \in U_{d} \cap V_{\alpha}$. In particular,

$$
S_{A}^{\alpha}=\psi_{\alpha}\left(A \cap V_{\alpha}, \mathbf{R}^{r_{2}}\right) \text { and } S_{A}=\cup_{\alpha} S_{A}^{\alpha}
$$

are semialgebraic. Since $f^{\prime}+E_{1}$ cross-sections the $M_{l}$ (see Proposition 3), $S_{A} \cap S_{B}$ $=\varnothing$ if $A \cap B=\varnothing$. If $M$ is a submanifold of $\left(f^{\prime}+E_{1}\right) \cap V_{\alpha} \cap U_{d}$ of dimension $<r_{1}$, then $\operatorname{dim}\left(M \times \mathbf{R}^{r_{2}}\right)<r_{1}+r_{2}$; hence

$$
\psi_{\alpha}\left(M \times \mathbf{R}^{r_{2}}\right)=\bigcup\left\{M_{l}: l \in M\right\} \text { has dimension }<r_{1}+r_{2}
$$

and is semialgebraic.

Consider any stratification $\mathscr{P}$ of $f^{\prime}+E_{1}$ compatible with the sets $A_{0}, U_{d} \cap\left(f^{\prime}\right.$ $\left.+E_{1}\right), V_{\alpha} \cap\left(f^{\prime}+E_{1}\right)$, and the given set $A$. Clearly $\operatorname{dim}\left(A_{0} \backslash A\right)<\operatorname{dim} A_{0}=r_{1}=$ $\operatorname{dim} E_{1}$ since $A$ is open and dense in $f^{\prime}+E_{1}$, and semialgebraic. If $B \in \mathscr{P}$ and 
$B \subseteq A_{0} \backslash A$, let $\alpha$ be the index such that $B \subseteq V_{\alpha}$. Now $\operatorname{dim} B<r_{1}$ and $\psi_{\alpha}$ is differentiable, so $S_{B}=\bigcup\left\{M_{l}: l \in B\right\}=\psi_{\alpha}\left(B \times \mathbf{R}^{r_{2}}\right)$ must have $\operatorname{dim} S_{B} \leqslant \operatorname{dim} B+$ $r_{2}<r_{1}+r_{2}$. Next, consider any stratification of $f^{\prime}+\mathfrak{f}^{\perp}$ compatible with $S_{A}, S_{A_{0}}$. Recall that $\operatorname{dim} \mathfrak{f}^{\perp}=r_{1}+r_{2}$. As $S_{A_{0}}=U_{d} \cap\left(f^{\prime}+\mathfrak{t}^{\perp}\right)$ is Zariski-open,

$$
\operatorname{dim}\left(f^{\prime}+\mathfrak{f}^{\perp}\right) \backslash S_{A_{0}}<r_{1}+r_{2} ;
$$

also, $S_{A_{0}} \backslash S_{A}=S_{A_{0} \backslash A}=\bigcup\left\{S_{B}: B \in \mathscr{P}, B \subseteq A_{0} \backslash A\right\}$ has dimension $<r_{1}+r_{2}$ by the preceding remarks. This proves (12).

The map $\phi^{\sim}: U_{e}^{\sim} \cap\left(f^{\perp}+\mathfrak{f}^{\perp}\right) \rightarrow \Sigma^{\chi}$ is constant on the varieties $M_{l}$. We have shown (Corollary to Proposition 3) that the $M_{l}$ are transverse to $f^{\prime}+E_{1}$ in the set-theoretic sense; this is also true for the tangent spaces, by part (e) of the Corollary. For each Zariski-open set $V_{\alpha} \subseteq \mathrm{g}^{*}$ we now restrict the map $\psi_{\alpha}$ to a (rational nonsingular) map $\psi_{\alpha}:\left(V_{\alpha} \cap E^{*}\right) \times \mathbf{R}^{r_{2}} \rightarrow \mathrm{g}^{*}$. Then $\operatorname{range}\left(\psi_{\alpha}\right) \subseteq U_{e}^{-} \cap$ $\left(f^{\perp}+\mathfrak{f}^{\perp}\right) \cap U_{d}$ and the composite $\phi^{\sim} \circ \psi_{\alpha}:\left(V_{\alpha} \cap E^{*}\right) \times \mathbf{R}^{r_{2}} \rightarrow \mathrm{g}^{*}$ is rational nonsingular and constant on the sets $(l) \times \mathbf{R}^{r_{2}}$; hence it is determined by its restriction to $\left(V_{\alpha^{\circ}} \cap E^{*}\right) \times(0)$, where it is equal to $\phi \circ \psi_{\alpha}$. Since $\psi_{\alpha}\left(l, \mathbf{R}^{r_{2}}\right)=M_{l}$ and tangent spaces are transverse if $l \in V_{\alpha} \cap E^{*}$, we see that

$$
\operatorname{rank}\left(d \psi_{\alpha}\right)_{(l, 0)}=r_{1}+r_{2}=\operatorname{dim} \mathfrak{A}^{\perp}, \quad l \in V_{\alpha} \cap E^{*} .
$$

This remains true on an open set containing $\left(V_{\alpha} \cap E^{*}\right) \times(0)$, and $\psi_{\alpha}$ is a local diffeomorphism on it. For $(l, t)$ in this set we have

$$
\begin{aligned}
\operatorname{rank}\left(d \phi^{\sim}\right)_{\psi_{\alpha}(l, t)} & =\operatorname{rank} d\left(\phi^{\sim} \circ \psi_{\alpha}\right)_{(l, t)} \\
& =\operatorname{rank} d\left(\phi^{\circ} \psi_{\alpha}\right)_{(l, 0)}=\operatorname{rank} d \phi_{l} .
\end{aligned}
$$

But generic (maximal) rank of $d \phi^{\sim}, d \phi$ is achieved on Zariski-open subsets of $U_{e}^{-} \cap\left(f^{\prime}+\mathfrak{f}^{\perp}\right), E^{*}$ so we conclude that

$$
\text { generic } \operatorname{rank}\left\{d \phi_{l} \tilde{l}: l \in f^{\prime}+\mathfrak{H}^{\perp}\right\}=k .
$$

This proves (a) and shows that $\Sigma^{x}=\operatorname{range}\left(\phi^{\sim}\right)$ has dimension $k$.

If $\Sigma^{x} \backslash \Sigma^{*}$ contains a $k$-dimensional piece $S$, then $S$ is open in $\Sigma^{x}$ (relative topology) and $\phi^{-1}(S)$ is open in $U_{e}^{\sim} \cap\left(f^{\prime}+\mathfrak{l}^{\perp}\right)$. By (12), $S_{E^{*}}=$ $\cup\left\{M_{l}: l \in E^{*}\right\}$ is open, dense in $f^{\prime}+\mathfrak{H}^{\perp}$; hence the set

$$
S^{\prime}=\phi^{-1}(S) \cap U_{d} \cap\left(f^{\prime}+\mathfrak{k}^{\perp}\right) \cap S_{E^{*}}
$$

is nonempty and open in $f^{\prime}+\mathfrak{f}^{\perp}$. Clearly, $U\left\{M_{l}: l \in S^{\prime}\right\}$ is equal to $S^{\prime}$. Thus $S^{\prime}$ meets $E^{*}$ in a nonempty set. Since $S \supseteq \phi^{\sim}\left(S^{\prime}\right) \supseteq \phi\left(S^{\prime} \cap E^{*}\right), S$ meets $\Sigma^{*}=$ $\phi\left(E^{*}\right)$, which is a contradiction. This proves (b).

Splitting $E^{*}=E_{r}^{*} \cup E_{s}^{*}$ as at the outset of this proof, we first note that $\operatorname{dim}\left(f^{\prime}+E_{1}\right) \backslash E^{*}<r_{1}=\operatorname{dim} E_{1}$; furthermore, $\operatorname{dim} E^{*} \backslash E_{r}^{*}<r_{1}$. [In fact, if the latter were not true, there would be an open piece in $E^{*} \backslash E_{r}^{*}$ which would contain a rectangular coordinate patch $N$ adapted to the $\phi$-foliation of $E^{*}$. Then $\phi(N) \subseteq$ $\phi\left(E_{s}^{*}\right)=\Sigma_{s}^{*}$ would contain a $k$-dimensional set, in conflict with the definitions.] Thus the decomposition of Theorem 2 gives

$$
\rho=\operatorname{Ind}_{K \rightarrow G}(\chi) \cong \int_{E_{r^{*}}^{*}}^{\oplus} \pi_{f^{\prime}+l} d l .
$$


Stratify $\Sigma_{r}^{*}$ into $k$-dimensional pieces, which are open in $\Sigma^{*}$; cover $E_{r}^{*}$ with rectangular coordinate patches $N_{j}=I_{j}^{1} \times I_{j}^{2} \subseteq \mathbf{R}^{k} \times \mathbf{R}^{r_{1}-k}$ adapted to the $\phi$-foliation of $E^{*}$, such that each $\phi\left(N_{j}\right)=F_{j}$ lies in a single piece of $\Sigma_{r}^{*}$. Let $G_{j}=$ $F_{j} \backslash\left(\cup_{i<j} F_{i}\right)$; these sets partition $\Sigma_{r}^{*}$, and since $\phi$ is constant on each fiber $(s) \times I_{j}^{2} \subseteq N_{j}$, the sets $M_{j}=\phi^{-1}\left(G_{j}\right) \cap N_{j}$ are rectangular, of the form $K_{j} \times I_{j}^{2}$. Since $\phi$ is a diffeomorphism of $I_{j}^{1} \times(0)$ to an open set in $\Sigma_{r}^{*}$, it carries $k$-dimensional Euclidean measure $m_{j}$ on $I_{j}^{1} \times(0)$ to a measure equivalent to the $k$-volume $\nu_{j}=\nu \mid \phi\left(N_{j}\right)$. Thus we get

$$
\begin{aligned}
\int_{M_{j}}^{\oplus} \pi_{l} d \lambda & \cong \int_{K_{j} \times I_{j}^{2}}^{\oplus} \pi_{(s, t)} d m_{j}(s) d t \\
& \cong \int_{K_{j}}^{\oplus} \infty \cdot \pi_{(s, 0)} d m_{j}(s) \cong \int_{G_{j}}^{\oplus} \infty \cdot \pi_{l^{\prime}} d \nu\left(l^{\prime}\right)
\end{aligned}
$$

and

$$
\rho \cong \int_{f^{\prime}+E_{1}}^{\oplus} \pi_{l} d l \cong \int_{\Sigma_{r}^{*}}^{\oplus} \infty \cdot \pi_{l^{\prime}} d \nu\left(l^{\prime}\right) \cong \int_{\Sigma^{x}}^{\oplus} \infty \cdot \pi_{l^{\prime}} d \nu\left(l^{\prime}\right)
$$

5. We continue with the notation of $\$ 4$ and now treat the remaining case, when $\tau_{0}=0$. Then $k=\operatorname{rank} d \phi_{l}=r_{1}=\operatorname{dim} E_{1}$ and $\phi$ is a local diffeomorphism from $E^{*}$ into $V_{T(e)}$. As we shall see, the multiplicities are finite and bounded in this case. We will also give a geometric description of $\tau_{0}$.

For $l^{\prime} \in \Sigma_{r}^{*}$ let $A\left(l^{\prime}\right)=G \cdot l^{\prime} \cap E_{r}^{*}=G \cdot l^{\prime} \cap E^{*}$ and $B\left(l^{\prime}\right)=\operatorname{card} A\left(l^{\prime}\right)$.

THEOREM 4. Let the hypotheses be as in Theorem 3, but now assume $\tau_{0}=0$. Then

(a) There is a finite number $B$ such that $B\left(l^{\prime}\right) \leqslant B$ for all $l^{\prime} \in \Sigma_{r}^{*}$.

(b) If we let $\Sigma_{r}^{*}(j)=\left\{l^{\prime} \in \Sigma_{r}^{*}: \operatorname{card}\left(G \cdot l^{\prime} \cap E^{*}\right)=j\right\}$, this set is semialgebraic in $V_{T(e)}$, as is

$$
E_{r}^{*}(j)=\phi^{-1}\left(\Sigma_{r}^{*}(j)\right) \cap E_{r}^{*}=\bigcup\left\{G \cdot l^{\prime} \cap E_{r}^{*}: l^{\prime} \in \Sigma_{r}^{*}(j)\right\} .
$$

(c) If $[\nu]$ is the canonical measure class on $\Sigma_{r}^{*}$, then

$$
\begin{aligned}
\operatorname{Ind}_{K \rightarrow G}(\chi) & \cong \bigoplus_{j=1}^{B} \int_{\Sigma_{r}^{*}(j)}^{\oplus} j \cdot \pi_{l^{\prime}} d \nu\left(l^{\prime}\right) \\
& \cong \int_{\Sigma^{x}}^{\oplus} n\left(l^{\prime}\right) \pi_{l^{\prime}} d \nu\left(l^{\prime}\right)
\end{aligned}
$$

where

$$
n\left(l^{\prime}\right)=\operatorname{card}\left(G \cdot l^{\prime} \cap\left(f^{\prime}+E_{1}\right)\right), \quad \text { all } l^{\prime} \in \Sigma^{\chi} .
$$

Proof. From the discussion of Theorem 1, the set $\Sigma_{e}^{\sim}=U_{e}^{\sim} \cap V_{T(e)}$ is semialgebraic and there is a birational nonsingular map $P_{e}: \Sigma_{e}^{\sim} \times V_{S(e)} \rightarrow U_{e}^{\sim}$ such that $P_{e}\left(l^{\prime}, \cdot\right)$ is a polynomial diffeomorphism from $\mathbf{R}^{q}$ to the $\mathrm{Ad}^{*}(G)$-orbit of $l^{\prime}$ for each $l^{\prime} \in \Sigma_{e}^{\sim}$, if we identify $V_{S(e)} \cong \mathbf{R}^{q}$. Let $l_{1}, \ldots, l_{n}$ be a basis of $\mathrm{g}^{*}$ such that $l_{1}, \ldots, l_{p}$ is a basis for $E_{1}$. In these coordinates the map has the form

$$
F:\left(t_{1}, \ldots, t_{q}\right) \rightarrow \sum_{j=1}^{n} P_{j}\left(t_{1}, \ldots, t_{q} ; l^{\prime}\right) l_{j}
$$


where $P_{j}$ is a polynomial in the $t_{j}$. Now $E^{*}$, as defined in (9), is Zariski-open in $f^{\prime}+E_{1}$; since $E_{r}^{*}, E_{s}^{*}$ are pullbacks in $E^{*}$ of $\Sigma_{r}^{*}, \Sigma_{s}^{*}$, an orbit $G \cdot l^{\prime}$ for $l^{\prime} \in \Sigma_{r}^{*}$ hits $E_{r}^{*} \Leftrightarrow$ it hits $E^{*}$. The number of points in $G \cdot l^{\prime} \cap E^{*}$ is equal to the number of roots of the polynomial map

$$
(s, t) \rightarrow F(t)-\left(f^{\prime}+\sum_{i=1}^{p} s_{i} l_{i}\right), \quad s \in \mathbf{R}^{p}, t \in \mathbf{R}^{q},
$$

lying in the Zariski-open set $B \times \mathbf{R}^{q}$, where $B=\left\{s: f^{\prime}+\sum_{i=1}^{p} s_{i} l_{i} \in E^{*}\right\}$. Now we use the following result, whose proof we sketch. We reduce to the scalar polynomial case discussed in this lemma by considering the sum of squares of components of the polynomial map given above.

Lemma 1. Let $Z \subseteq \mathbf{R}^{n}$ be a Zariski-open set, $Z=\left\{x \in \mathbf{R}^{n}: Q(x) \neq 0\right\}$ for some polynomial $Q$, and let $P: \mathbf{R}^{n} \rightarrow \mathbf{R}$ be a polynomial, $P=P\left(t_{1}, \ldots, t_{n}\right)$. Then there is a number $N$ depending only on $m, n, \operatorname{deg} P$, and $\operatorname{deg} Q$ such that: either $P(x)=0$ has a one-parameter family of solutions in $Z$, or the number of solutions in $Z$ is bounded by $N$.

Proof. If $Z=\mathbf{R}^{n}$, we may argue by induction on $n$ using Sturm's theorem. If $n=1$, the number of roots is bounded by $\operatorname{deg} P$, or else every $x$ is a root. In general, write $P$ in the form

$$
P=\sum_{j=0}^{r} Q_{j}\left(t_{1}, \ldots, t_{n-1}\right) t_{n}^{j} .
$$

For fixed $t_{1}, \ldots, t_{n-1}$, this has a solution if and only if (i) the $Q_{j}$ are all zero, or (ii) the conditions of Sturm's theorem are met, see [15]. The latter involve certain algebraic inequalities in the $Q_{j}\left(t_{1}, \ldots, t_{n-1}\right)$; if they are satisfied on any open interval in $t_{n}$, we get a 1-parameter family of solutions. If not, we have a bunch of polynomial equations in $t_{1}, \ldots, t_{n-1}$ which are satisfied if and only if there is a $t_{n}$ making $Q\left(t_{1}, \ldots, t_{n}\right)=0$. The number of such $t_{n}$ is $\leqslant r$, and we have now reduced the number of variables to be considered by one.

If $Z \neq \mathbf{R}^{n}$ we note that there is a continuous one-to-one correspondence between the solutions of $P(x)=0, x \in Z$, and those of

$$
P(x)=0, \quad Q(x) y=1 \quad(y \in \mathbf{R}) .
$$

This proves (a).

For (b), the statement that $l^{\prime} \in \Sigma_{r}^{*}(j)$ can be written as the statement that a certain set of polynomial equations and inequalities have solutions. For example, if $j=1$ then $l^{\prime} \notin \Sigma_{r}^{*}(1)$ if and only if

(i) $l^{\prime} \in \Sigma_{r}^{*}$, and

(ii) $\sum_{j=1}^{p} P_{j}\left(t, l^{\prime}\right)^{2}=0$ has a solution $t=\left(t_{1}, \ldots, t_{q}\right)$,

(iii) $\sum_{j=1}^{p} P_{j}\left(t, l^{\prime}\right)^{2}=0, \sum_{j=1}^{p} P_{j}\left(u, l^{\prime}\right)^{2}=0, \sum_{j=1}^{q}\left(t_{j}-u_{j}\right)^{2}>0$ has a solution $(t, u)$.

The Tarski-Seidenberg theorem says that this set is semialgebraic, and by taking complements, so is $\Sigma_{r}^{*}(1)$. The proof for $\Sigma_{r}^{*}(j)$ is similar. This proves (b). 
In (c) we have

$$
\text { Ind }_{K \rightarrow G}(\chi)=\bigoplus_{j=1}^{B} \int_{E_{r}^{*}(j)}^{\oplus} \pi_{l} d l \text {. }
$$

We want to write this as a direct integral over the $k$-dimensional pieces of $\Sigma_{r}^{*}(j)$. Fix a stratification of $\Sigma_{r}^{*}$ compatible with the $\Sigma_{r}^{*}(j)$; let $\left\{\Sigma_{j}^{\alpha}\right\}$ be the $k$-dimensional pieces of $\Sigma_{r}^{*}(j)$, if any, and let $\Sigma_{r}^{* *}(j)==\bigcup_{\alpha} \Sigma_{j}^{\alpha}, \Sigma_{r}^{* *}=\bigcup_{j} \Sigma_{r}^{* *}(j)$. Let

$$
\begin{aligned}
& E_{r}^{* *}(j)=\phi^{-1}\left(\Sigma_{r}^{* *}(j)\right) \cap E^{*}=\bigcup\left\{A\left(l^{\prime}\right): l^{\prime} \in \Sigma_{r}^{* *}(j)\right\}, \\
& E^{* *}=\bigcup_{j} E_{r}^{* *}(j)=\phi^{-1}\left(\Sigma_{r}^{* *}\right) \cap E^{*} .
\end{aligned}
$$

Then $E^{* *}$ is open in $f^{\prime}+E_{1}$, since $k$-dimensional pieces are open in $\Sigma_{r}^{*}$, and it is obvious that $\operatorname{dim} \Sigma^{*} \backslash \Sigma_{r}^{* *}<k$. Furthermore, $\operatorname{dim} E^{*} \backslash E^{* *}<k$. [If not, there would be an (open) $k$-dimensional piece in $E^{*} \backslash E^{* *}$, but then $\phi\left(E^{*} \backslash E^{* *}\right) \subseteq$ $\Sigma^{*} \backslash \Sigma_{r}^{* *}$ would contain a piece of dimension $k$, which is a contradiction.]

Split $E^{* *}$ into its (open) topologically connected components, $E^{* *}=\cup_{\beta} E^{\beta}$. Each of these $k$-dimensional manifolds is mapped into a single $\Sigma_{j}^{\alpha}$ by $\phi$, which is an open mapping. For each $\Sigma_{j}^{\alpha}$ consider the $E^{\beta}$ which map into it; if $x \in \Sigma_{j}^{\alpha}$, for each such $E^{\beta}$ let

$$
m_{\beta}(x)=\operatorname{card}\left\{y \in E^{\beta}: \phi(y)=x\right\} .
$$

This covering index has integer values, and $\Sigma_{\beta} m_{\beta}(x)=j$ for all $x \in \Sigma_{j}^{\alpha}$. Furthermore, if $x_{0} \in \Sigma_{j}^{\alpha}$ is fixed, then for each $\beta$ there is a neighborhood of $x_{0}$ on which $m_{\beta}(x) \geqslant m\left(x_{0}\right)$. Let $I=\left\{\beta: m_{\beta}\left(x_{0}\right) \neq 0\right\}$; this is finite, and the preceding inequality holds throughout some neighborhood $N$ of $x_{0}$ for all $\beta \in I$. Then for all $x \in N$ we have

$$
j=\sum_{\beta} m_{\beta}\left(x_{0}\right)=\sum_{\beta \in I} m_{\beta}\left(x_{0}\right) \leqslant \sum_{\beta \in I} m_{\beta}(x) \leqslant \sum_{\beta} m_{\beta}(x)=j .
$$

Thus the $m_{\beta}$ are constant on $N$, and only finitely many are nonzero. In particular, each $m_{\beta}$ is locally constant on $\Sigma_{j}^{\alpha}$ (hence constant), only finitely many can be nonzero (hence there are only finitely many $E^{\beta}$ ), $\phi: E^{\beta} \rightarrow \Sigma_{j}^{\alpha}$ is a covering map with uniform covering index $m_{\beta}$, and $\sum m_{\beta}=j$.

Let $\nu_{j, \alpha}$ be any $k$-dimensional volume on the manifold $\Sigma_{j}^{\alpha}$ and let $\nu_{j}=\Sigma \nu_{j, \alpha}$ on $\Sigma_{r}^{* *}(j)$. Then

$$
\int_{E^{\beta}}^{\oplus} \pi_{l} d l=\sum_{\Sigma_{j}^{\alpha}}^{\oplus} m_{\beta} \cdot \pi_{l^{\prime}} d \nu_{j, \alpha}\left(l^{\prime}\right) \quad \text { all } \alpha, \beta, j
$$

and since $\sum m_{\beta}=j$, we get

$$
\rho \cong \int_{E^{* *}}^{\oplus} \pi_{l} d l=\bigoplus_{j=1}^{B} \int_{\Sigma_{r}^{* *}(j)}^{\oplus} j \cdot \pi_{l^{\prime}} d \nu_{j}\left(l^{\prime}\right) .
$$

Since $\operatorname{dim} \Sigma_{r}^{*}(j) \backslash \Sigma_{r}^{* *}(j)<k$, this set is $\nu$-null and we get the first direct integral in (c). This may be rewritten as

$$
\int_{\Sigma_{r}^{*}}^{\oplus} n_{1}\left(l^{\prime}\right) \pi_{l^{\prime}} d \nu\left(l^{\prime}\right)
$$


where $n_{1}\left(l^{\prime}\right)=\operatorname{card}\left(G \cdot l^{\prime} \cap E_{r}^{*}\right)$. To get the second, more canonical, version we must show that for $\nu$-a.e. $l^{\prime}$ in $\Sigma_{r}^{*}, G \cdot l^{\prime}$ cannot meet $f^{\prime}+E_{1}$ outside $E_{r}^{*}$. Then we can replace $\Sigma_{r}^{*}$ by $\Sigma^{x}$ since $\operatorname{dim} \Sigma^{x} \backslash \Sigma_{r}^{*}<k$, as shown in Theorem 3 .

Let $S_{1}=U_{e} \sim \cap\left(f^{\prime}+E_{1}\right)$ and $\Sigma^{1}=\phi\left(S_{1}\right)$. We showed that $\operatorname{dim} \Sigma^{1} \backslash \Sigma_{r}^{*}<k$; it is obvious that $\operatorname{dim} S^{1} \backslash E^{*}<k$, and in proving Theorem 3 we showed that $\operatorname{dim} E^{*} \backslash E_{r}^{*}<k$, so the set $X=S_{1} \backslash E_{r}^{*}$ has $\operatorname{dim} X<k$. This implies that $Y=$ $\phi(X)$ has $\operatorname{dim} Y<k$, hence $\operatorname{dim} Y \cap \Sigma_{r}^{*}<k$. Let $W=\Sigma_{r}^{*} \backslash Y$. This set obviously has full measure in $\Sigma_{r}^{*}$ and $\Sigma^{x}$. But if $l^{\prime} \in W$, we have $G \cdot l^{\prime} \cap X=\varnothing$. On the other hand, we always have $G \cdot l^{\prime} \cap\left(f^{\prime}+E_{1}\right) \subseteq U_{e}^{-} \cap\left(f^{\prime}+E_{1}\right)=S_{1}$, which implies that $G \cdot l^{\prime} \cap E_{r}^{*}=G \cdot l^{\prime} \cap\left(f^{\prime}+E_{1}\right)$. This completes the proof.

To get a more canonical version of Theorem 4(c) we must show that the multiplicity function there satisfies

$$
n\left(l^{\prime}\right)=\text { number of } \operatorname{Ad}^{*}(K) \text {-orbits in } G \cdot l^{\prime} \cap\left(f^{\prime}+\mathfrak{f}^{\perp}\right)
$$

for $\nu$-a.e. $l^{\prime}$ in $\Sigma^{x}$. To begin this we need a technical result.

In the proof we must consider all the sets $U_{d}, d \in \mathscr{D}$, discussed in Proposition 2 (defined relative to a chain of subalgebras $\mathfrak{f}=\mathfrak{g}_{0} \subseteq \cdots \subseteq \mathfrak{g}_{m}=\mathfrak{g}$ ) and the set $U_{e} \sim$ defined in Theorem 3 (relative to a Mal'cev basis in $g$ ). We assume the subalgebras and Mal'cev basis are fixed, and let $d=$ largest index such that $U_{d}$ meets $f^{\prime}+\mathfrak{f}^{\perp}$; the splitting $\mathrm{f}^{\perp}=E_{1} \oplus E_{2}$ is defined relative to this index $d$. Proposition 3 will play a central role.

Proposition 4. Let $\tau_{0}=0$, define $\Sigma^{* *}, \Sigma_{r}^{* *}(j), E^{* *}, E_{r}^{* *}(j)$ as in Theorem 4, let $k=$ generic rank of $\phi^{\sim}: U_{e}^{\sim} \cap\left(f^{\prime}+\mathfrak{l}^{\perp}\right) \rightarrow V_{T(e)}$ (recall Theorem 3(a)), and define

$$
\begin{aligned}
& U=\left\{l \in U_{e}^{\sim} \cap\left(f^{\prime}+\mathfrak{f}^{\perp}\right): \operatorname{rank} d \phi_{l}^{\sim}=k\right\}, \\
& W=\left\{l \in U_{e}^{\sim} \cap\left(f^{\prime}+\mathfrak{H}^{\perp}\right): \operatorname{dim} \operatorname{Ad}^{*}(K) l=r\right\}
\end{aligned}
$$

( where $r=$ generic $\operatorname{dim} \operatorname{Ad}^{*}(K) l$ for $l \in f^{\prime}+\mathfrak{k}^{\perp}$ )

$$
E^{0}=E^{* *} \cap U \cap W \quad\left(E^{* *}=\cup_{j} E_{r}^{* *}(j)\right)
$$

(recall $E^{* *} \subseteq E^{*} \subseteq\left(f^{\prime}+E_{1}\right) \cap U_{e}^{-} \cap U_{d} \cap \mathcal{J} ; E^{*}$ is nonempty and Zariski-open in $f^{\prime}+E_{1}$, and $E^{* *}$ is open, dense, and semialgebraic)

$$
F=\left(U_{e}^{-} \cap\left(f^{\prime}+\mathfrak{H}^{\perp}\right)\right) \backslash \operatorname{Ad}^{*}(K) E^{0} .
$$

Then

(a) $r$ is equal to $r_{2}=\operatorname{dim} E_{2}$.

(b) $W$ is an $\operatorname{Ad}^{*}(K)$-invariant Zariski-open set in $f^{\prime}+\mathfrak{H}^{\perp}$.

(c) For each $l \in U_{d} \cap U \cap W$, the intersection of $\mathcal{O}_{l}$, with this set is a relatively closed manifold, and $\operatorname{Ad}^{*}(K) l=M_{l}=$ connected component of $\mathcal{O}_{l} \cap U_{d} \cap U \cap W$ through $l$.

(d) $E^{0}$ is a nonempty, open, dense semialgebraic set in $f^{\prime}+E_{1}$ and $\operatorname{Ad}^{*}(K) E^{0}$ is semialgebraic, contains a dense open subset of $f^{\prime}+\mathfrak{H}^{\perp}$, and has complement of measure zero. 
(e) The set $F$ is semialgebraic, as are its $\phi^{\sim}$-image, and G-saturant $[F]=$ $\phi^{--1}\left(\phi^{-}(F)\right)=\bigcup\left\{G \cdot l \cap U_{e}^{\sim} \cap\left(f^{\prime}+\mathfrak{l}^{\perp}\right): l \in F\right\}$. The saturant is a finite union of manifolds of dimension $<\operatorname{dim} \mathfrak{f}^{\perp}$, and hence has measure zero in $f^{\prime}+\mathfrak{H}^{\perp}$. All pieces in any stratification of the $\phi^{\sim}$-image have dimension $<k$.

(f) The set $H=\operatorname{Ad}^{*}(K) E^{0} \backslash[F]$ is equal to $\left(U_{e}^{\sim} \cap\left(f^{\prime}+\mathfrak{f}^{\perp}\right)\right) \backslash[F]$, is $G$ saturated, has complement of measure zero in $f^{\prime}+\mathfrak{H}^{\perp}$, and its $\phi^{\sim}$-image lies within $\Sigma^{* *}=\bigcup_{j} \Sigma_{r}^{* *}(j)$ of Theorem 4. Furthomore, for all $j$ and $l \in H$ :

$$
\begin{aligned}
& \phi^{\sim}(l) \in \sum_{r}^{* *}(j) \Leftrightarrow \mathcal{O}_{1} \cap\left(f^{\prime}+\mathfrak{H}^{\perp}\right) \text { consists of exactly } j \\
& \operatorname{Ad}^{*}(K) \text {-orbits }(\text { each having dimension } r) .
\end{aligned}
$$

If we let

$$
\begin{aligned}
H_{j} & =\phi^{--1}\left(\Sigma_{r}^{* *}(j)\right) \cap H \\
& =\left\{l \in H: \mathcal{O}, \cap\left(f^{\prime}+\mathfrak{H}^{\perp}\right) \text { consists of } j \operatorname{Ad}^{*}(K) \text {-orbits }\right\}
\end{aligned}
$$

for $1 \leqslant j \leqslant B$, then these sets are semialgebraic and partition $H$. Furthermore,

$$
\begin{aligned}
& \nu\left(\Sigma_{r}^{* *}(j) \backslash \phi^{\sim}\left(H_{j}\right)\right)=0, \quad 1 \leqslant j \leqslant B, \\
& \nu\left(\Sigma^{x} \backslash \phi^{\sim}(H)\right)=\nu\left(\Sigma^{x} \backslash \Sigma^{* *}\right)=0,
\end{aligned}
$$

and

$$
\begin{aligned}
\rho & \cong \bigoplus_{j=1}^{B} \int_{\phi^{-}\left(H_{j}\right)}^{\oplus} j \pi_{l^{\prime}} d \nu\left(l^{\prime}\right) \\
& \cong \int_{\phi^{-}(H)}^{\oplus} j \pi_{l^{\prime}} d \nu\left(l^{\prime}\right) \\
& \cong \int_{\phi^{-}(H)}^{\oplus} n_{1}\left(l^{\prime}\right) \pi_{l^{\prime}} d \nu\left(l^{\prime}\right)
\end{aligned}
$$

where $n_{1}\left(l^{\prime}\right)=$ number of $\operatorname{Ad}^{*}(K)$-orbits in $G \cdot l^{\prime} \cap\left(f^{\prime}+\mathfrak{l}^{\perp}\right)$, for $l^{\prime} \in \Sigma^{x}$.

Note 1 . The important technical point here lies in (14). Starting with Theorem 4 it is fairly easy to describe multiplicities as the number of $\operatorname{Ad}^{*}(K)$-orbits in $\mathcal{O}_{1} \cap S$ where $S$ is some computed set; (15) shows how to compute them as the number of $\operatorname{Ad}^{*}(K)$-orbits in $\mathcal{O}, \cap\left(f^{\prime}+\mathfrak{f}^{\perp}\right)$, a more natural task. This is the reason for constructing $H$.

Note 2. It is not hard to check that $U_{d}, U$, and $W$ are $\operatorname{Ad}^{*}(K)$-invariant. Since $H$ is $G$-saturated, it too is $\operatorname{Ad}^{*}(K)$-invariant, so that

$$
\begin{aligned}
\mathcal{O}_{l} \cap\left(f^{\prime}+\mathfrak{H}^{\perp}\right) & \subseteq \mathcal{O}_{l} \cap H \subseteq \mathcal{O}_{l} \cap \operatorname{Ad}^{*}(K) E^{0} \\
& \subseteq \mathcal{O}_{l} \cap U_{d} \cap U \cap W \subseteq \mathcal{O}_{l} \cap\left(f^{\prime}+\mathfrak{H}^{\perp}\right)
\end{aligned}
$$

for all $l \in H$. By (c), $\mathcal{O}_{1} \cap\left(f^{\prime}+\mathfrak{f}^{\perp}\right)$ is then a closed submanifold for all $l \in H$. This intersection lies in $\operatorname{Ad}^{*}(K) E^{0}$, which is a union of $\operatorname{Ad}^{*}(K)$-orbits, and consists of finitely many such orbits. Hence

If $l \in H, \mathcal{O}, \cap\left(f^{\prime}+\mathfrak{H}^{\perp}\right)$ is a closed submanifold and its connected components are precisely the $\operatorname{Ad}^{*}(K)$-orbits in it. 
This gives an alternative geometric description of multiplicities-just replace "Ad* $(K)$-orbits" with "connected components" in (15).

Proof. The set $U$ is Zariski-open in $f^{\prime}+\mathfrak{H}^{\perp}$. Since rank $\phi^{\sim}=k$ on $U$, there is a foliation of $U$ consistent with $\phi^{-}$: obviously the leaf through $l \in U$ coincides with the orbit intersection $\mathcal{O}_{1} \cap U$ locally; in particular each intersection $\mathcal{O}_{I} \cap U$ is a closed submanifold of $U$ and the leaf is the connected component of $\mathcal{O}_{1} \cap U$ containing $l$. Since $\tau_{0}=r_{1}-k=0$, the leaf dimension is $\operatorname{dim} \mathcal{H}^{\perp}-k=r_{2}$. For $l \in U_{d} \cap U$, the subspace $T_{l}=\operatorname{ad}^{*}(\mathrm{~g}) l \cap \mathfrak{f}^{\perp}$ is the component of the tangent space $\operatorname{ad}^{*}(\mathfrak{g}) l$ to $\mathcal{O}$, that is parallel to $\mathfrak{f}^{\perp}$; it includes $\operatorname{ad}^{*}(\mathfrak{f}) l$. Minimum dimension $\delta_{l}=\operatorname{dim} T_{l}$ (maximal transversality to $\mathfrak{f}^{\perp}$ ) is generic, so $\delta_{l}=\delta=\min \delta_{l}$ on some Zariski-open set $U_{1} \subseteq f^{\prime}+\mathfrak{l}^{\perp}$. In view of the following lemma we may determine the value of $\delta$ :

$$
\delta=\delta_{l}=\operatorname{corank}(d \tilde{\phi})_{l}=\operatorname{dim} \operatorname{ker}(d \tilde{\phi})_{l} \quad \text { all } l \in U_{1} .
$$

Lemma 2 (TRANSVERSAlity OF ORBITS). Let $U_{e}^{\sim}$ be one of the layers in the orbit parametrization of $\mathrm{g}^{*}$, as in Theorem 1. Let $P_{e}^{-1}: U_{e}^{\sim} \rightarrow \Sigma_{e} \times V_{S(e)}$ be the parametrizing map and $\pi_{S}, \pi_{T}$ the projections of $\mathrm{g}^{*}$ onto $V_{S(e)}, V_{T(e)}$. Let $S$ be an affine subspace of $\mathrm{g}^{*}$ that meets $U_{e}^{-}$in a Zariski-open subset $S^{\prime}$, and let $\phi=$ $\pi_{T} \circ P_{e}^{-1} \mid S^{\prime}: S^{\prime} \rightarrow V_{T(e)}$. Let $S^{\prime \prime}=\left\{l \in S^{\prime}: \operatorname{rank}(d \phi)\right.$, is maximal $\}$. For $l \in S^{\prime \prime}$ we have $\operatorname{ker}(d \phi)_{l}=\operatorname{ad}^{*}(\mathrm{~g}) l \cap T_{l} S$ where $T_{l} S$ is the tangent space to $S$ at $l$.

Proof. Since $d \phi$ has constant rank, there is a foliation of $S^{\prime \prime}$ whose leaves are components of orbit intersections with $S^{\prime \prime}$. In particular, $\operatorname{ker}\left(d \phi_{l}\right)=$ (leaf tangent space at $l) \subseteq \operatorname{ad}^{*}(\mathrm{~g}) l \cap T_{l} S$ for $l \in S^{\prime \prime}$. If for some $l \in S^{n}$ the inclusion is proper, let us choose an origin in $S$, a transverse subspace $W$, and decompose $\mathrm{g}^{*}=S \oplus W$ with corresponding projections $P_{S}$ and $P_{W}$. Then there is a $C^{\infty}$ curve $\mu(t)$ such that

$$
\begin{gathered}
\mu(0)=l, \quad \mu(t) \in \operatorname{Ad}^{*}(G) l \text { for all } t, \\
\mu^{\prime}(0) \in\left(\operatorname{ad}^{*}(\mathrm{~g}) l \cap T_{l} S\right) \backslash \operatorname{ker}(d \phi) l .
\end{gathered}
$$

By the last condition, $P_{W}(\mu(t))=O\left(t^{2}\right)$. In $\mathfrak{g}^{*}$ we have

$$
\mu^{\prime}(0)=\lim _{t \rightarrow 0} \frac{\mu(t)-\mu(0)}{t}=\lim _{t \rightarrow 0} \frac{P_{S}(\mu(t))-P_{S}(\mu(0))}{t}=\left(P_{S} \mu\right)^{\prime}(0) .
$$

The parametrizing map $P_{e}: \Sigma_{e}^{-} \times V_{S(e)} \rightarrow U_{e}^{\sim}$ of Theorem 1 has rational, nonsingular inverse $P_{e}^{-1}$ : that is, there are rational, nonsingular maps $Q_{\theta}$ on Zariski-open sets $U_{\theta} \subseteq \mathrm{g}^{*}$ that cover $U_{e}^{-}$and have the property $P_{e}^{-1}=Q_{\theta}$ on $U_{\theta} \cap U_{e}^{-}$for all $\theta$. Pick an index $\theta$ such that $l \in U_{\theta} \cap U_{e}^{\sim}$ and write $Q=Q_{\theta}$ for simplicity. Then $\mu(t) \in G \cdot l \subseteq U_{e}^{-} \cap U_{\theta}$ and

$$
\pi_{T} \circ Q(\mu(t))=\pi_{T} \circ Q(l)=\phi(l)
$$


for all $t$ near $t=0$. Since $\pi_{T} \circ Q$ is differentiable on $\mathrm{g}^{*}$ at $l$, being a nonsingular rational function on $U_{\theta}$, and $\phi=\pi_{T} \circ Q^{-1} \mid S \cap U_{e}^{\sim}$, we get

$$
\begin{aligned}
d \phi\left(\mu^{\prime}(0)\right) & =\left(\phi \circ P_{S} \mu\right)^{\prime}(0)=\lim _{t \rightarrow 0} \frac{\phi\left(P_{S} \mu(t)\right)-\phi(l)}{t} \\
& =\lim _{t \rightarrow 0} \frac{\pi_{T} \circ Q\left(P_{S} \mu(t)\right)-\pi_{T} \circ Q(\mu(t))}{t}+\lim _{t \rightarrow 0} \frac{\pi_{T} \circ Q(\mu(t))-\phi(l)}{t} \\
& =\lim _{t \rightarrow 0} \frac{\pi_{T} \circ Q\left(P_{S} \mu(t)\right)-\pi_{T} \circ Q(\mu(t))}{t}+0 \\
& =\lim _{t \rightarrow 0} \frac{O\left(t^{2}\right)}{t}=0 .
\end{aligned}
$$

But $\mu^{\prime}(0) \notin \operatorname{ker}(d \phi)$, by hypothesis.

By Theorem 3(a),

$$
\text { gen } \operatorname{rank}\left\{d \phi_{l}^{\tilde{I}}: l \in f^{\prime}+\mathfrak{H}^{\perp}\right\}=\operatorname{gen} \operatorname{rank}\left\{d \phi_{l}: l \in f^{\prime}+E_{1}\right\},
$$

so when $\tau_{0}=0$ we have, for generic $l \in f^{\prime}+\mathfrak{H}^{\perp}$,

$$
\begin{aligned}
\delta & =\operatorname{corank} d \phi_{l}^{\tilde{}}=\operatorname{dim} \mathfrak{l}^{\perp}-\operatorname{rank} d \phi_{l}^{\tilde{l}} \\
& =\operatorname{dim} \mathfrak{l}^{\perp}-\operatorname{rank} d \phi_{l}=\operatorname{dim} \mathfrak{l}^{\perp}-r_{1}=r_{2} .
\end{aligned}
$$

Once we know this, we may compute gen $\operatorname{dim} \operatorname{Ad}^{*}(K) l$ as follows. If $\mathfrak{r}_{l}=$ radical $=$ $\left\{X \in \mathrm{g}: \operatorname{ad}^{*}(X) l=0\right\}$, then $\operatorname{dim} \operatorname{ad}^{*}(\mathrm{~g}) l=\operatorname{dim} \mathrm{r}_{l^{\perp}}$, so for any $l \in \mathrm{g}^{*}$ we get

$$
\begin{aligned}
\delta_{l} & =\operatorname{dim}\left(\operatorname{ad}^{*}(\mathfrak{g}) l \cap \mathfrak{f}^{\perp}\right)=\operatorname{dim}\left(\mathfrak{r}_{l}^{\perp} \cap \mathfrak{H}^{\perp}\right)=\operatorname{dim}\left(\mathrm{r}_{l}+\mathfrak{t}\right)^{\perp} \\
& =\operatorname{dim} \mathfrak{g}-\operatorname{dim}\left(\mathfrak{r}_{l}+\mathfrak{H}\right) \\
& =\operatorname{dim} \mathfrak{g}-\operatorname{dim} \mathfrak{f}-\operatorname{dim} \mathrm{r}_{l}+\operatorname{dim}\left(\mathfrak{H} \cap \mathfrak{r}_{l}\right) \\
& =\operatorname{dim} \mathcal{O}_{l}-\operatorname{dim} \mathrm{Ad}^{*}(K) l .
\end{aligned}
$$

For $l \in U_{d} \cap\left(f^{\prime}+\mathfrak{H}^{\perp}\right)$ we have $\operatorname{dim} \mathcal{O}_{l}=2 r_{2}$, and on some smaller Zariski-open set we also have $\delta_{l}=\delta=r_{2}$. Thus, when $\tau_{0}=0$, we get

$$
\operatorname{dim} \operatorname{Ad}^{*}(K) l=r_{2} \quad \text { for generic } l \in f^{\prime}+\mathfrak{H}^{\perp}
$$

so $r=r_{2}$. This proves (a).

Clearly $W$ is $\operatorname{Ad}^{*}(K)$-invariant and Zariski-open since $\operatorname{dim} \operatorname{Ad}^{*}(K) l=$ $\operatorname{dim} \operatorname{ad}^{*}(\mathfrak{f}) l$ maximal is a Zariski-open condition for $l \in f^{\prime}+\mathfrak{H}^{\perp}$. Also, $U$ is $\operatorname{Ad}^{*}(K)$-invariant since $\operatorname{Ad}^{*}(K)$ is a diffeomorphism of $f^{\prime}+\mathfrak{l}$ for each $k \in K$. Furthermore, it is not hard to check that $U_{d}$ is $\mathrm{Ad}^{*}(K)$-invariant. Now consider any $l \in U_{d} \cap U \cap W$. We have

$$
\mathcal{O}_{1} \cap U \text { locally equal to } M_{l} \text {, with dimension } r_{2} \text {, }
$$

$\operatorname{Ad}^{*}(K) l$ locally contained in $\mathcal{O}_{l} \cap U$, with dimension $r_{2}$.

So all these sets coincide locally near $l$. But $\mathrm{Ad}^{*}(K) l$ and $M_{l}$ are algebraic-graphs of rational functions-hence they are equal. Since $\operatorname{Ad}^{*}(K) l \subseteq U_{d} \cap U \cap W$ by invariance of these sets,

$$
\operatorname{Ad}^{*}(K) l=M_{l}=\text { connected component of } l \text { in } \mathcal{O}_{l} \cap U_{d} \cap U \cap W
$$


and (b), (c) are proved. Note that we have also shown

$$
U_{d} \cap U \cap W \text { is the union of all } M_{l} \text { in } U_{d} \text { that meet it. }
$$

Now consider $E^{0}=E^{* *} \cap U \cap W$. Since $E^{* *}$ is dense, open, and semialgebraic in $f^{\prime}+E_{1}$, (12) implies that $S=\bigcup\left\{M_{l}: l \in E^{* *}\right\}$ contains a dense open set in $f^{\prime}+\mathfrak{f}^{\perp}$. On the other hand, $U_{d} \cap U \cap W$ is Zariski-open in $f^{\prime}+\mathfrak{H}^{\perp}$ and, as we have just seen, is the union of the $M_{l}$ it meets. If $l_{0} \in S \cap U_{d} \cap U \cap W$, then $M_{l_{0}}$ meets $f^{\prime}+E_{1}$ (by Theorem 2), so the set $E^{* *} \cap U \cap W$ is nonempty. But $U \cap W$ must then meet $f^{\prime}+E_{1}$ in a nonempty Zariski-open set. Thus $E^{0}$ is dense, open, and semialgebraic in $f^{\prime}+E_{1}$. From (12) and (20a) we conclude that $S^{0}=\bigcup\left\{M_{l}: l\right.$ $\left.\in E^{0}\right\}=\operatorname{Ad}^{*}(K) E^{0}$ contains an open dense set of $f^{\prime}+\mathfrak{H}^{\perp}$ whose complement has measure zero. It is obviously semialgebraic, since $\operatorname{Ad}^{*}(K)$ acts polynomially, so (d) is proved.

Now consider the set $F$. It is obviously semialgebraic, as are $\tilde{\phi}(F)$ and the saturant $[F]=\tilde{\phi}^{-1}(\tilde{\phi}(F))$. Once we show that the pieces in any stratification of $\tilde{\phi}(F)$ have dimension $<k$ it follows that the pieces in any stratification of $[F]$ have dimension $<r_{1}+r_{2}$. In fact if there is a piece of $[F]$ with dimension $r_{1}+r_{2}$ it is open in $f^{\prime}+\mathfrak{f}^{\perp}$ and rank $\tilde{\phi}=k$ on some open subset of it which maps to a $k$-dimensional manifold within $\tilde{\phi}(F)$.

Let $\mathscr{S}^{-}$be a stratification of $\Sigma^{\chi}$ compatible with the sets $\tilde{\phi}\left(E^{0}\right)=$ $\tilde{\phi}\left(\operatorname{Ad}^{*}(K) E^{0}\right)$ and $\tilde{\phi}(F)$. The maximum dimension of pieces in $\mathscr{S}^{\sim}$ is $k=r_{1}$. Suppose there is a piece $M_{0} \widetilde{\subseteq} \tilde{\phi}(F)$ with $\operatorname{dim} M_{0}{ }^{\sim}=k$; we will produce a contradiction. Take any stratification $\mathscr{S}$ of $f^{\prime}+\mathfrak{f}^{\perp}$ compatible with the sets $U_{e}^{-} \cap\left(f^{\prime}\right.$ $\left.+\mathfrak{f}^{\perp}\right)$, the $U_{d_{1}} \cap\left(f^{\prime}+\mathfrak{H}^{\perp}\right)\left(d_{i} \in \mathscr{D}\right), \operatorname{Ad}^{*}(K) E^{0}, \tilde{\phi}^{-1}\left(\mathscr{S}^{\sim}\right)$, and $U$. The set $M_{0}^{\sim}$ is covered by $\tilde{\phi}$-images of pieces $M_{0} \in \mathscr{S}$ lying in $F$. At least one $M_{0}$ must meet (hence lie within) the set $U$ where $\operatorname{rank}\left(d \tilde{\phi}_{l}\right)=k$. In fact, for any $l \in U_{e}^{\sim} \cap\left(f^{\prime}+\right.$ $\left.\mathfrak{f}^{\perp}\right), \operatorname{rank}\left(\tilde{\phi} \mid M_{0}\right)_{l} \leqslant \operatorname{rank}(\tilde{\phi})_{l} \leqslant k$ and if equality fails for every $l \in M_{0}$ and every piece $M_{0}$ we are in conflict with the fact that $\operatorname{dim} M_{0}{ }^{-}=k$ and $M_{0}{ }^{-}=$ $\bigcup\left\{\tilde{\phi}\left(M_{0}\right): M_{0} \subseteq F\right\}$. Take any $M_{0}$ such that $\operatorname{rank}\left(\tilde{\phi} \mid M_{0}\right)_{l}=k$ on some open subset $A \subseteq M_{0}$; clearly $A \subseteq U$. The tangent spaces $\left(T M_{0}\right)_{l}, l \in A$, contain subspaces of dimension $k$ transverse to the leaves of the $\tilde{\phi}$-foliation of $U$. (This is of course the maximum possible degree of transversality.) Thus there exists a submanifold $M \subseteq A$ $\subseteq M_{0}$ of dimension $k$ such that $\tilde{\phi} \mid M$ is a diffeomorphism into $M_{0} \tilde{}$.

Next consider the $U_{d_{i}}\left(d_{i} \in \mathscr{D}\right)$. The index $d=d_{0}$ is the first such that $U_{d_{i}}$ meets $f^{\prime}+\mathfrak{\perp}$. There may be other indices $d_{0}>d_{1}>\cdots>d_{p}$ such that $U_{d^{\prime}}$ meets $f^{\prime}+\mathfrak{H}^{\perp}$. Now $U_{e}^{-} \cap\left(f^{\prime}+\mathfrak{H}^{\perp}\right)$ is covered by the sets $U_{d_{0}}, \ldots, U_{d_{p}}\left(d_{i} \in \mathscr{D}\right)$. Let $U_{d_{i}}$ be the first of these meeting $M$ and recall that the sets $V_{d}=U_{d^{\prime}}>d U_{d^{\prime}}$ are Zariski-open in $\mathrm{g}^{*}$. Since $M \cap V_{d}=\varnothing$ for $d>d_{i}, U_{d_{i}} \cap M$ must contain a nonempty open subset of $M$; replacing $M$ with this set, we may assume $M \subseteq U_{d_{i}}$. By Proposition 3, for each $l \in M$ the variety $M_{l}$ in $U_{d_{i}}$ lies in the $\tilde{\phi}$-leaf through $l$ (since $M_{l} \subseteq \mathcal{O}_{l} \cap U_{d_{l}}$ ) and meets $M$ only at $l$, by transversality. We now claim that, owing to the way the $M_{l}$ were constructed,

The set $Y=\bigcup\left\{M_{l}: l \in M\right\}$ contains an open set in $f^{\prime}+\mathfrak{H}^{\perp}$. 
Once (21) has been proved we argue as follows: $Y$ must then meet the open, dense in set in $\operatorname{Ad}^{*}(K) E^{0} \subseteq U_{d} \cap U \cap W\left(d=d_{0}\right)$. This implies that $d_{i}=d_{0}=d$ because each $M_{l}\left(l \in U_{d_{i}}\right)$ is contained in $U_{d_{i}}$, which would otherwise be disjoint from $U_{d}$. We have seen in (c), (d) that $\operatorname{Ad}^{*}(K) E^{0}=\bigcup\left\{M_{l}: l \in E^{0}\right\}$, hence contains every $M_{l}$ in $U_{d}$ that meets it. Thus $M$ meets $\operatorname{Ad}^{*}(K) E^{0}$. However, $M \subseteq M_{0} \subseteq F$, which by definition is disjoint from $\operatorname{Ad}^{*}(K) E^{0}$, so the hypothesis that $\operatorname{dim} M_{0}{ }^{-}=k$ at last leads to a contradiction, and (e) is proved once we establish (21).

For (21) notice that $M$ lies within the set $A_{i}=U_{d_{i}} \cap U_{e}^{\sim} \cap U \cap\left(f^{\prime}+\mathfrak{f}^{\perp}\right)$. But $\operatorname{dim} \mathcal{O}_{1}$ is constant for $l \in U_{e}^{\sim}$, by definition, and $\operatorname{dim} \mathcal{O}_{l}=2 r_{2}\left(r_{2}=r_{2}\left(d_{0}\right)\right)$ for $l \in U_{e}^{-} \cap U_{d_{0}}$; since the latter set is nonempty we see that $\operatorname{dim} \mathcal{O}_{l}=2 r_{2}$ for $l \in U_{e}^{-}$. On the other hand, $\operatorname{dim} \mathcal{O}_{l}=2 r_{2}\left(d_{i}\right)$ if $l \in U_{d_{i}}$, so if $l \in A_{i}$ (a nonempty set) we have $\operatorname{dim} \mathcal{O}_{1}=2 r_{2}\left(d_{i}\right)=2 r_{2}$. Therefore $r_{2}\left(d_{i}\right)=r_{2}$.

Fix a point $l \in M$ and take a centered rectangular coordinate neighborhood $N=I_{1} \times I_{2}$ compatible with the $\phi^{\sim}$-foliation of $U \subseteq M$. Then $\phi^{\sim}$ is a diffeomorphism on $I_{1} \times(0)$ and is constant on leaves $(s) \times I_{2}$, which have dimension = corank $d \phi^{\sim}=k=r_{2}$ because $\tau_{0}=0$. If $l^{\prime} \in N \cap U_{d_{i}}, M_{l^{\prime}}$ is a smooth manifold with dimension $r_{2}\left(d_{i}\right)=r_{2}(d)=r_{2}$, and is constant locally in the leaf $(s) \times I_{2}$ through $l^{\prime}$. Hence $M_{l^{\prime}} \cap N$ contains $(s) \times I_{2}$. As above, $M \subseteq A_{i} \subseteq U_{d_{i}} \cap U$ and is transverse to the $\phi^{\sim}$-foliation. Reduce the size of $M$ so that $M \subseteq N$ and the projection $P_{1}: M \rightarrow I_{1} \times(0)$ is a diffeomorphism. Then $\bigcup\left\{M_{l^{\prime}}: l^{\prime} \in M\right\} \supseteq P_{1}(M)$ $\times I_{2}$. The latter is an open set in $N$, which is open in $U$ and in $f^{\prime}+\mathfrak{I}^{\perp}$. This proves the claim (21) and (e).

For (f) it is clear that the two descriptions of $H$ agree; $G$-saturation is obvious from one of them. Also, $H \subseteq \operatorname{Ad}^{*}(K) E^{0} \subseteq U_{d}$ since $E^{0} \subseteq U_{d}$ and $U_{d}$ is $\operatorname{Ad}^{*}(K)$-invariant. Because $H$ is $G$-saturated, $H \cap\left(f^{\prime}+E_{1}\right) \subseteq E_{0} \subseteq E^{* *}$ and $H \cap\left(f^{\prime}+E_{1}\right)=H \cap E^{* *}$. Furthermore,

$$
H=\bigcup\left\{M_{l}: l \in H \cap\left(f^{\prime}+E_{1}\right)\right\}=\bigcup\left\{\operatorname{Ad}^{*}(K) l: l \in H \cap E^{* *}\right\},
$$

and $\tilde{\phi}(H) \subseteq \Sigma^{* *}$. For $l \in H$,

$$
\tilde{\phi}(l) \in \Sigma_{r}^{* *}(j) \Leftrightarrow \operatorname{card}\left(\mathcal{O}, \cap E^{* *}\right)=j .
$$

But by $G$-saturation of $H$,

$$
\begin{aligned}
\operatorname{card}\left(\mathcal{O}, \cap E^{* *}\right) & =\operatorname{card}\left(\mathcal{O}_{l} \cap E^{0}\right)=\text { number of } \operatorname{Ad}^{*}(K) \text {-orbits in } \mathcal{O}_{l} \cap H \\
& =\text { number of } K \text {-orbits in } \mathcal{O}_{l} \cap\left(f^{\prime}+\mathfrak{H}^{\perp}\right) .
\end{aligned}
$$

So, (14) is proved. For the rest of the statements, the sets $H, H_{j}$ are semialgebraic, as are the sets $\phi^{\sim}\left(H_{j}\right)$; clearly $\phi^{\sim}\left(H_{j}\right) \subseteq \Sigma_{r}^{* *}(j)$. Take any stratification $\mathscr{S}$ of $\Sigma^{x}$ compatible with the sets $\Sigma_{r}^{* *}(j), \phi^{\sim}\left(H_{j}\right)$. Maximal dimension of pieces $M_{0}^{\sim} \in \mathscr{S}$ is $r_{1}$. All pieces in $\Sigma^{\chi} \backslash \Sigma_{r}^{* *}$ have dimension $<, r_{1}$, and likewise for pieces in $\Sigma_{r}^{* *}(j) \backslash \phi^{-}\left(H_{j}\right)$; otherwise, $\operatorname{dim} M_{0}^{\sim}=r_{1}$ and $\tilde{\phi}^{-1}\left(M_{0}^{\sim}\right)$ is open in $f^{\prime}+\mathfrak{H}^{\perp}$ and disjoint from $H$, which is a contradiction. The first and second lines of (15) now follow from Theorem 4, since $\nu\left(\Sigma_{r}^{* *}(j) \backslash \phi^{\sim}\left(H_{j}\right)\right)=0$. For $l^{\prime} \in \phi^{\sim}\left(H_{j}\right), n_{1}\left(l^{\prime}\right)=j$, but by Theorem $4(\mathrm{c})$ we have $j=n\left(l^{\prime}\right)=\operatorname{card}\left(G \cdot l^{\prime} \cap\left(f^{\prime}+E_{1}\right)\right)$ for $\nu$-a.e. $l^{\prime} \in$ $\Sigma_{r}^{*}(j)$. Since

$$
\nu\left(\Sigma_{r}^{*}(j) \backslash \Sigma_{r}^{* *}(j)\right)=\nu\left(\Sigma_{r}^{* *}(j) \backslash \phi^{-}\left(H_{j}\right)\right)=0, \quad 1 \leqslant j \leqslant B,
$$

we get the final formula in (15), and this completes the proof of the Proposition. 
We now state the final version of the multiplicity theorem for the case $\tau_{0}=0$. If $\operatorname{dim} \chi=1$, and we define $\tau_{0}$ as in (10), it is immediate from the preceding discussion. The case $\operatorname{dim} \chi>1$ and the task of reconciling definitions (10) and (22) of $\tau_{0}$ will be taken up below. The case $\tau_{0}>0$ is covered in Theorem 3 .

THEOREM 5. Let $\mathfrak{g}$ be a nilpotent Lie algebra, $\mathfrak{f}$ a subalgebra, and $G, K$ the simply connected Lie groups. Let $P: \mathrm{g}^{*} \rightarrow \mathfrak{l}^{*}$ be the natural projection, let $\chi \in K^{\wedge}$ be associated with a K-orbit $\mathcal{O}_{\chi} \subseteq \mathfrak{1}^{*}$, and form the variety $P^{-1}\left(\mathcal{O}_{\chi}\right) \subseteq \mathrm{g}^{*}$. Using a Mal'cev basis for $\mathfrak{g}$, define layers $\left\{U_{e}^{\sim}: e \in \mathscr{E}\right\}$, let $U_{e}^{\sim}$ be the first layer that meets $P^{-1}\left(\mathcal{O}_{\chi}\right)$, and define parametrization map $P_{e}: \Sigma_{e}^{\sim} \times V_{S(e)} \rightarrow U_{e}^{\sim}$ as usual. Let

$$
\Sigma^{x}=\pi_{T} \circ P_{e}^{-1}\left(U_{e}^{\sim} \cap P^{-1}\left(\mathcal{O}_{\chi}\right)\right),
$$

$[\nu]$ the canonical measure class, and define $\tau_{0}$ to be the generic value of

$$
\operatorname{dim} G \cdot l-2 \operatorname{dim} K \cdot l+\operatorname{dim} \mathcal{O}_{\chi}, \quad l \in P^{-1}\left(\mathcal{O}_{\chi}\right) .
$$

Then if $\tau_{0}=0$, we have

$$
\rho \cong \int_{\Sigma^{x}}^{\oplus} n_{1}\left(l^{\prime}\right) \pi_{l^{\prime}} d \nu\left(l^{\prime}\right)
$$

where

$$
n_{1}\left(l^{\prime}\right)=\text { number of } \operatorname{Ad}^{*}(K) \text {-orbits in } G \cdot l^{\prime} \cap P^{-1}\left(\mathcal{O}_{\chi}\right)
$$

Furthermore,

(a) There is a bound $B$ such that $n_{1}\left(l^{\prime}\right) \leqslant B$, v-a.e. $l^{\prime}$.

(b) For $\nu$-a.e. $l^{\prime}$, the intersection is a closed manifold in $P^{-1}\left(\mathcal{O}_{\chi}\right)$, and its connected components are the $\operatorname{Ad}^{*}(K)$-orbits.

For now we shall compare (10) and (22) when $\operatorname{dim} \chi=1$; the general case will be derived from this in the next section. If $\operatorname{dim} \chi=1$, then $\chi=e^{2 \pi i f}$ and $P^{-1}\left(\mathcal{O}_{\chi}\right)=$ $P^{-1}(K \cdot f)=P^{-1}(f)=f^{\prime}+\mathfrak{f}^{\perp}$, where $f^{\prime}$ is any extension of $f$ to $\mathrm{g}$. Definition (10) uses the layering $\left\{U_{d}: d \in \mathscr{D}\right\}$ to obtain a splitting $\mathfrak{H}^{\perp}=E_{1} \oplus E_{2}$; then, with respect to a $\mathrm{Mal}^{\prime} \mathrm{cev}$ basis for $\mathrm{g}$, we define the layers $\left\{U_{e}^{\sim}: e \in \mathscr{E}\right\}$, determine the first layer $U_{e}^{\sim}$ that meets $f^{\prime}+\mathfrak{H}^{\perp}$, and define the parametrization map $\phi^{\sim}: U_{e}^{\sim} \cap$ $\left(f^{\prime}+\mathfrak{l}^{\perp}\right) \rightarrow \Sigma^{\chi}$ for this layer. Then in (10) we let

$$
\tau_{0}=\operatorname{dim} E_{1}-\text { gen } \operatorname{rank}\left\{d \phi_{l}: l \in f^{\prime}+E_{1}\right\}
$$

where $\phi=\phi^{\sim} \mid U_{e}^{\sim} \cap\left(f^{\prime}+E_{1}\right)$. In the splitting,

$$
r_{2}=\operatorname{dim} E_{2}=\frac{1}{2} \operatorname{dim} G \cdot l \quad \text { all } l \in U_{d} \cap\left(f^{\prime}+\mathfrak{l}^{\perp}\right)
$$

since dimensions jump by 2 for each basis vector in $E_{2}$, and $\operatorname{dim} K \cdot f=0$. Thus we have an interpretation of $r_{2}$ that is independent of the $U_{d}$ layering:

$$
r_{2}=\frac{1}{2} \operatorname{gen} \operatorname{dim}\left\{G \cdot l: l \in f^{\prime}+\mathfrak{H}^{\perp}\right\}, \quad r_{1}=\operatorname{dim} \mathfrak{H}^{\perp}-r_{2} .
$$

By Lemma 2 and formulas (17), (18), which are valid for any value of $\tau_{0}$,

$$
\operatorname{dim} \mathfrak{H}^{\perp}-\operatorname{rank} d \phi^{\sim}=\operatorname{dim}^{*}(g) l \cap \mathfrak{A d}^{\perp}=\operatorname{dim} G \cdot l-\operatorname{dim} \operatorname{Ad}^{*}(K) l
$$


for generic $l \in f^{\prime}+\mathfrak{H}^{\perp}$. Putting this together with Theorem 3(a), we get

$$
\begin{aligned}
\tau_{0} & =\operatorname{dim} \mathfrak{L}^{\perp}-r_{2}-\text { gen rank } d \phi_{l}^{\tilde{l}} \\
& =\operatorname{dim} \mathfrak{H}^{\perp}-\frac{1}{2} \operatorname{gen} \operatorname{dim} G \cdot l-\operatorname{dim} \mathfrak{H}^{\perp}+\operatorname{gen} \operatorname{dim} G \cdot l-g e n \operatorname{dim} K \cdot l \\
& =\frac{1}{2}\left[\text { gen } \operatorname{dim} G \cdot l-2 \operatorname{gen} \operatorname{dim} K \cdot l+\operatorname{dim} \mathcal{O}_{\chi}\right]
\end{aligned}
$$

for $l \in f^{\prime}+\mathfrak{l}^{\perp}$, since we are in the case $\operatorname{dim} \mathcal{O}_{\chi}=0$. Except for the factor $\frac{1}{2}$, this is the form of $\tau_{0}$ in (22). In the next section we show how to deal with the case $\tau_{0}>0$.

Note. Recall that, generically, $\operatorname{dim} G \cdot l=2 \operatorname{dim} E_{2}$. Hence for generic $l$, we have

$$
\tau_{0}=\operatorname{dim} G \cdot l-2 \operatorname{dim} K \cdot l=2\left(\operatorname{dim} E_{2}-\operatorname{dim} K \cdot l\right)=2\left(\operatorname{dim} M_{l}-\operatorname{dim} K \cdot l\right) .
$$

If $\tau_{0}>0$, then $\operatorname{dim} K \cdot l<\operatorname{dim} M_{l} \leqslant \operatorname{dimension}$ of the connected component of $l$ in $G \cdot l \cap P^{-1}\left(\mathcal{O}_{\chi}\right)$, so that $G \cdot l$ contains infinitely many $\operatorname{Ad}^{*}(K)$-orbits if $\tau_{0}>0$. That is, the multiplicity formula

$$
n(\pi)=\text { number of } \mathrm{Ad}^{*}(K) \text {-orbits in } \mathcal{O}_{\pi} \cap P^{-1}\left(\mathcal{O}_{\chi}\right)
$$

holds in the $\tau_{0}>0$ case as well. As Proposition 5 will show, this formula will also hold when $\operatorname{dim} \chi>1$.

6. We now remove the hypothesis that $\operatorname{dim} \chi=1$, and prove Theorem 5 in full generality. We continue with the notation of that theorem.

The multiplicity formula for $\rho=\operatorname{Ind}_{K \rightarrow G}(\chi), \chi \in K^{\wedge}$, follows essentially because $\chi$ is monomial. Thus if $\chi$ corresponds to an orbit $\mathcal{O}_{\chi} \subseteq \mathfrak{H}^{*}$, and $f \in \mathcal{O}_{\chi}$, we can find a maximal subordinate subalgebra $\mathfrak{k}_{0}$ for $f$. Then $\chi$ is induced from the 1-dimensional representation $\sigma=e^{2 \pi i f_{0}}, f_{0}=f \mid \mathfrak{f}_{0}$. Let $f^{\prime}$ be any extension of $f$ to g. the multiplicity formula (23) for $\rho=\operatorname{Ind}_{K \rightarrow G}(\sigma)$ is then a consequence of the following proposition. If $l \in \mathrm{g}^{*}$, we abbreviate $\operatorname{Ad}^{*}(H) l=H \cdot l$ if $H \subseteq G$, and similarly for $f \in \mathfrak{H}^{*}$. We have orbits $\mathcal{O}_{\sigma}=K_{0} \cdot f_{0}=\left\{f_{0}\right\} \subseteq \mathfrak{f}_{0}^{*}, \mathcal{O}_{\chi}=K \cdot f \subseteq \mathfrak{F}^{*}$, $\mathcal{O}=G \cdot f^{\prime} \subseteq \mathrm{g}^{*}$. Let $S$ be a smooth cross-section for $K_{0} \backslash K$, and let $P_{0}: \mathrm{g}^{*} \rightarrow \mathfrak{f}_{0}^{*}$, $P: \mathrm{g}^{*} \rightarrow \mathfrak{f}^{*}$ be the natural projections.

Proposition 5. Let $\mathcal{O} \subseteq \mathrm{g}^{*}$ be any $\mathrm{Ad}^{*}(G)$ orbit. Then $P^{-1}\left(\mathcal{O}_{\chi}\right) \supseteq P_{0}^{-1}\left(\mathcal{O}_{\sigma}\right)=$ $f^{\prime}+\mathfrak{H}^{\perp}$, and there is a bijection between $K$-orbits in $\mathcal{O} \cap P^{-1}\left(\mathcal{O}_{\chi}\right)$ and $K_{0}$-orbits in $\mathcal{O} \cap P_{0}^{-1}\left(\mathcal{O}_{\sigma}\right)$, given by $\Phi\left(K_{0} \cdot l\right)=K \cdot l$.

To prove this, we need a lemma.

LEMma 3. In the setting of Proposition 5, the extension $f \in \mathfrak{H}^{*}$ satisfies

(a) $\operatorname{Ad}^{*}\left(K_{0}\right) f=f^{\prime}+\mathfrak{H}_{0}^{\perp}, K$ where $\mathfrak{f}_{0}^{\perp, K}$ is the annihilator of $\mathfrak{f}_{0}$ in $\mathfrak{k}^{*}$.

(b) If $l \in \mathcal{O}_{\chi}=K \cdot f \subseteq \mathfrak{I}^{*}$, there is a unique $s \in S$ such that $\operatorname{Ad}^{*}(s) l \in f+\mathfrak{f}_{0}^{\perp, K}$.

Note. $K \cdot f$ need not be a union of cosets of $\mathfrak{f}_{0}^{+}, K$, since $K_{0}$ need not be normal in K.

Proof. (a) is a well-known fact: If $\mathfrak{m}$ is any polarization for $f \in \mathfrak{l}^{*}$, and $M=\exp (\mathrm{m})$, then $M \cdot f=f+\mathfrak{m}^{\perp, K}$.

(b) Since $K=K_{0} S$ there is some $k=k_{0} s\left(k_{0} \in K_{0}, s \in S\right)$ such that $\operatorname{Ad}^{*}(k) l=$ $f$. Then $\operatorname{Ad}^{*}(s) l \in f+\mathfrak{l}_{0}^{\perp}, K$, from (a). If $s_{1}, s_{2} \in S$ satisfy $\operatorname{Ad}^{*}\left(s_{i}\right) l \in f+\mathfrak{f}_{0}^{\perp}, K$ for $i=1,2$ then there exist $k_{1}, k_{2} \in K_{0}$ such that $l=\operatorname{Ad}^{*}\left(s_{i}^{-1} k_{i}\right) f$. Hence

$$
\operatorname{Ad}^{*}\left(k_{1}^{-1} s_{1} s_{2}^{-1} k_{2}\right) f=f \quad \text { and } \quad k_{1}^{-1} s_{1} s_{2}^{-1} k_{2} \in \operatorname{Stab}_{K}(f) \subseteq K_{0}
$$


since a polarization always contains the radical, or $K_{0} s_{1}=K_{0} s_{2}$. Thus $s_{1}=s_{2}$ and (b) follows.

Proof of Proposition 5. It is easy to check that $P^{-1}\left(\mathcal{O}_{\chi}\right)$ is $K$-invariant, $P_{0}^{-1}\left(\mathcal{O}_{\sigma}\right)$ is $K_{0}$-invariant, $P_{0}^{-1}\left(\mathcal{O}_{\sigma}\right)=P^{-1}\left(f^{\prime}+\mathfrak{f}_{0}^{\perp} \cdot K\right)=P^{-1}\left(K_{0} \cdot f\right) \subseteq P^{-1}\left(\mathcal{O}_{\chi}\right)$, and that

$$
\begin{gathered}
l \in \mathcal{O} \cap P_{0}^{-1}\left(\mathcal{O}_{\sigma}\right) \Rightarrow K_{0} \cdot l \subseteq \mathcal{O} \cap P_{0}^{-1}\left(\mathcal{O}_{\sigma}\right), \\
K \cdot l \subseteq \mathcal{O} \cap P^{-1}\left(\mathcal{O}_{\chi}\right) .
\end{gathered}
$$

Thus we may define a map $\Phi$ from $K_{0}$-orbits in $\mathcal{O} \cap P_{0}^{-1}\left(\mathcal{O}_{\sigma}\right)$ to $K$-orbits in $\mathcal{O} \cap P^{-1}\left(\mathcal{O}_{\chi}\right)$ by $\Phi\left(K_{0} \cdot l\right)=K \cdot l$.

This map is surjective, for if $l_{1} \in P^{-1}\left(\mathcal{O}_{\chi}\right)$, the lemma says we can find an $s \in S$ such that $l=\operatorname{Ad}^{*}(s) l_{1}$ satisfies $l \mid \mathfrak{f}=f$ and hence $l \mid \mathfrak{f}_{0}=f_{0}$. Thus $l \in \mathcal{O} \cap P_{0}^{-1}\left(\mathcal{O}_{\sigma}\right)$ and $l_{1} \in K \cdot l=\Phi\left(K_{0} l\right)$. Finally, $\Phi$ is injective, for if $K \cdot l_{1}=K \cdot l$ with $l_{1}, l \in \mathcal{O}$ $\cap P_{0}^{-1}\left(\mathcal{O}_{\sigma}\right)$, then there exists a $k \in K$ with $l_{1}=\operatorname{Ad}^{*}(k) l$. Write $k=k_{0}^{-1} s\left(k_{0} \in K_{0}\right.$, $s \in S)$ and let $l_{2}=\operatorname{Ad}^{*}\left(k_{0}\right) l_{1}=\operatorname{Ad}^{*}(s) l$. Then $l, l_{1}, l_{2} \in \mathcal{O} \cap P_{0}^{-1}\left(\mathcal{O}_{\sigma}\right)$. But $l^{\prime} \in$ $P_{0}^{-1}\left(\mathcal{O}_{\sigma}\right) \Leftrightarrow l^{\prime}\left|\mathfrak{f}_{0}=f_{0} \Leftrightarrow l^{\prime}\right| \mathfrak{f} \in f^{\prime}+\mathfrak{H}_{0}^{\perp}, K$; both $\operatorname{Ad}^{*}(s) l=\operatorname{Ad}^{*}\left(k_{0}\right) l_{1}$ and $l$ restrict to elements in $f^{\prime}+\mathfrak{f}_{0}^{\perp}, K$ on $\mathrm{f}$. By the uniqueness condition (b) of the lemma, $s=e$. Hence $\operatorname{Ad}^{*}\left(k_{0}\right) l_{1}=l$, or $K_{0} \cdot l=K_{0} \cdot l_{1}$.

Proof of THEOREM 5. The result has been proved when $\operatorname{dim} \chi=1$, as was the geometric interpretation of $\tau_{0}$. In general, Proposition 5 ensures that every $K$-orbit in $P^{-1}\left(\mathcal{O}_{\chi}\right)$ meets $P_{0}^{-1}\left(\mathcal{O}_{\sigma}\right)$. Thus $U_{e}^{-}$is also the first layer in $\mathrm{g}^{*}$ that meets $P_{0}^{-1}\left(\mathcal{O}_{\sigma}\right)=$ $f^{\prime}+\mathfrak{f}_{0}^{\perp}$, and

$$
\Sigma^{\chi}=\phi^{\sim}\left(U_{e}^{\sim} \cap P^{-1}\left(\mathcal{O}_{\chi}\right)\right)=\phi^{\sim}\left(U_{e}^{\sim} \cap\left(f^{\prime}+\mathfrak{f}_{0}^{\perp}\right)\right)
$$

is the same set for $\left(K_{0}, \sigma\right)$ and $(K, \chi)$. In particular, the generic dimension of $G \cdot l$ is the same whether we consider ponts in $f^{\prime}+\mathfrak{f}_{0}^{\perp}$ or $P^{-1}\left(\mathcal{O}_{\chi}\right)$; it is the dimension of all orbits in $U_{e}^{\sim}$. Theorem 5 applies to the pair $\left(K_{0}, \sigma\right)$; by Proposition 5 , the multiplicity formula (23) is the same whether we consider $\left(K_{0}, \sigma\right)$ or $(K, \chi)$, and (a) is also clear. As for (b): for $\nu$-a.e. $l \in \Sigma^{x}, G \cdot l \cap\left(f^{\prime}+\mathfrak{f}_{0}^{\perp}\right)$ is a closed submanifold consisting of finitely many $K_{0}$-orbits. These correspond one-to-one with the $K$-orbits in $G \cdot l \cap P^{-1}\left(\mathcal{O}_{\chi}\right)$, hence the latter is a closed submanifold whose connected components are the $K$-orbits. This proves (b).

Next, we compare the value of $\tau_{0}$ in these two situations; they are equal. In fact, if $\mathfrak{h}$ is a Lie algebra let us denote the stabilizer of $l \in \mathfrak{h} *$ by $\mathfrak{h}_{l}$. We must compare

$$
\text { gen } \operatorname{dim} K \cdot l-\frac{1}{2} \operatorname{dim} \mathcal{O}_{\chi}=\operatorname{gen} \operatorname{dim} K \cdot l-\frac{1}{2} \operatorname{dim} K \cdot f \quad\left(l \in P^{-1}\left(\mathcal{O}_{\chi}\right)\right)
$$
with

$$
\text { gen } \operatorname{dim} K_{0} \cdot l-\frac{1}{2} \operatorname{dim} \mathcal{O}_{\sigma}=\operatorname{gen} \operatorname{dim} K_{0} \cdot l \quad\left(l \in P_{0}^{-1}\left(\mathcal{O}_{\sigma}\right)=f^{\prime}+\mathfrak{f}_{0}^{\perp}\right) .
$$

But the maps $P, P_{0}$ are equivariant for the actions of $\operatorname{ad}^{*}(\mathfrak{f}), \operatorname{ad}^{*}\left(\mathfrak{f}_{0}\right)$ respectively. Thus if $l \in f^{\prime}+\mathfrak{f}_{0}^{\perp}, P(l) \in P\left(f^{\prime}+\mathfrak{f}_{0}^{\perp}\right)=f+\mathfrak{f}_{0}^{\perp}, K=K_{0} \cdot f$, so there is a $k_{0} \in K_{0}$ such that $P\left(k_{0} \cdot l\right)=f$. Thus $K_{0} \cdot l$ has a representative in $f^{\prime}+\mathfrak{f}^{\perp}$ and gen $\operatorname{dim} K_{0} \cdot l\left(\right.$ for $\left.l \in f^{\prime}+\mathfrak{f}_{0}^{\perp}\right)=\operatorname{gen} \operatorname{dim} K_{0} \cdot l\left(\right.$ for $\left.l \in f^{\prime}+\mathfrak{f}^{\perp}\right)$.

Likewise, if $l \in P^{-1}\left(\mathcal{O}_{\chi}\right)$, there is a $k \in K$ such that $k \cdot l \in f^{\prime}+\mathfrak{f}_{0}^{\perp}$ (Proposition 5), and hence a $k^{\prime} \in K$ such that $k^{\prime} \cdot l \in f^{\prime}+\mathfrak{f}^{\perp}$. Thus

gen $\operatorname{dim} K \cdot l\left(\right.$ for $\left.l \in P^{-1}\left(\mathcal{O}_{\chi}\right)\right)=\operatorname{gen} \operatorname{dim} K \cdot l\left(\right.$ for $\left.l \in f^{\prime}+\mathfrak{f}{ }^{\perp}\right)$. 
Now fix an $l \in f^{\prime}+\mathfrak{f}^{\perp}$. If $X \in \mathfrak{f} \cap \mathfrak{g}_{l}$, this implies that $X \in \mathfrak{f}$, ad* $(X) l=0 \Rightarrow$ $\operatorname{ad}^{*}(X) P l=\operatorname{ad}^{*}(X) f=0 \Rightarrow X \in \mathfrak{f}_{f} \subseteq \mathfrak{f}_{0} \subseteq \mathfrak{f}$ since $\mathfrak{f}_{0}$ is a polarization for $f$. Hence $\mathfrak{g}_{l} \cap \mathfrak{f} \subseteq \mathfrak{g}_{l} \cap \mathfrak{f}_{f} \subseteq \mathfrak{g}_{l} \cap \mathfrak{f}_{0} \cap \mathfrak{f}$ and these subalgebras are equal. Now

$$
\operatorname{dim} K \cdot l=\operatorname{dim} \mathfrak{f}-\operatorname{dim} \mathfrak{f} \cap \mathfrak{g}_{l}=\operatorname{dim} \mathfrak{f}-\operatorname{dim} \mathfrak{f}_{f} \cap \mathfrak{g}_{l}
$$

and since $\operatorname{dim} \mathfrak{f}_{0}=\frac{1}{2}\left(\operatorname{dim} \mathfrak{f}+\operatorname{dim} \mathfrak{f}_{f}\right)$, we get

$$
\begin{aligned}
\operatorname{dim} K \cdot l-\frac{1}{2} \operatorname{dim} K \cdot f & =\operatorname{dim} \mathfrak{f}-\operatorname{dim} \mathfrak{f}_{f} \cap \mathfrak{g}_{l}-\frac{1}{2}\left(\operatorname{dim} \mathfrak{f}-\operatorname{dim} \mathfrak{f}_{f}\right) \\
& =\frac{1}{2} \operatorname{dim} \mathfrak{f}-\operatorname{dim} \mathfrak{f}_{f} \cap \mathfrak{g}_{l}+\frac{1}{2} \operatorname{dim} \mathfrak{f}_{f} \\
& =\frac{1}{2}\left(\operatorname{dim} \mathfrak{f}+\operatorname{dim} \mathfrak{f}_{f}\right)-\operatorname{dim} \mathfrak{f}_{f} \cap \mathfrak{g}_{l} \\
& =\operatorname{dim} \mathfrak{f}_{0}-\operatorname{dim} \mathfrak{f}_{0} \cap \mathfrak{g}_{l}=\operatorname{dim} K_{0} \cdot l .
\end{aligned}
$$

Write $X$ for $P^{-1}\left(\mathcal{O}_{\chi}\right)$. If we compute $\tau_{0}$ for $(K, \chi)$ we have

$$
\begin{aligned}
\tau_{0} & =\frac{1}{2} \text { gen } \operatorname{dim}\left\{\mathcal{O}_{l}: l \in X\right\}-\text { gen } \operatorname{dim}\{K \cdot l: l \in X\}+\frac{1}{2} \operatorname{dim} \mathcal{O}_{\chi} \\
& =\frac{1}{2} \operatorname{gen} \operatorname{dim}\left\{\mathcal{O}_{l}: l \in X\right\}-\text { gen } \operatorname{dim}\left\{K_{0} \cdot l: l \in f^{\prime}+\mathfrak{f}^{\perp}\right\} \\
& =\frac{1}{2} \operatorname{gen} \operatorname{dim}\left\{\mathcal{O}_{l}: l \in X\right\}-\text { gen } \operatorname{dim}\left\{K_{0} \cdot l: l \in f^{\prime}+\mathfrak{f}_{0}^{\perp}\right\} \\
& =\frac{1}{2} \text { gen } \operatorname{dim}\left\{\mathcal{O}_{l}: l \in f^{\prime}+\mathfrak{H}_{0}^{\perp}\right\}-\text { gen } \operatorname{dim}\left\{K_{0} \cdot l: l \in f^{\prime}+\mathfrak{f}_{0}^{\perp}\right\}+\operatorname{dim} \mathcal{O}_{\sigma} .
\end{aligned}
$$

But the last line is just $\tau_{0}$ computed for $\left(K_{0}, \sigma\right)$, as required.

Theorem 5 has geometric elegance, but in doing calculations it may be helpful to note that multiplicities can be computed by considering intersections of $G$-orbits $\mathcal{O}_{\text {, }}$ with a flat variety $f^{\prime}+\mathfrak{l}^{\perp}$, rather than the variety $P^{-1}\left(\mathcal{O}_{\chi}\right)$. Here we take $f \in \mathcal{O}_{\chi}, f^{\prime}$ any extension of $f$ to $\mathrm{g}$. However, $f^{\prime}+\mathfrak{f}^{\perp}$ need not be $\operatorname{Ad}^{*}(K)$-invariant, so in the situation described below the multiplicity can only be described as the number of connected components in $\mathcal{O}_{1} \cap\left(f^{\prime}+\mathfrak{H}^{\perp}\right)$, and the connection with $K$-orbits is less apparent. The following result from [8] follows easily from Theorem 5.

THEOREM 6. Let notation be as in Theorem 5. If $\tau_{0}>0$, then

$$
\rho \cong \int_{\Sigma^{x}}^{\oplus} \infty \cdot \pi_{l^{\prime}} d \nu\left(l^{\prime}\right)
$$

If $\tau_{0}=0$, let $f \in \mathcal{O}_{\chi}$ be fixed and let $f^{\prime}$ be any extension to $\mathrm{g}$. Then

$$
\rho \cong \int_{\Sigma^{x}}^{\oplus} m\left(l^{\prime}\right) \pi_{l^{\prime}} d \nu\left(l^{\prime}\right)
$$

where

$$
m\left(l^{\prime}\right)=\text { number of connected components in } G \cdot l^{\prime} \cap\left(\dot{f}^{\prime}+\mathfrak{l}^{\perp}\right) .
$$

Furthermore, if $\tau_{0}=0$,

(a) There is a bound $B$ such that $m\left(l^{\prime}\right) \leqslant B$ for $\nu$-a.e. $l^{\prime}$.

(b) For v-a.e. $l^{\prime}$, the intersection is a closed submanifold in $\left(f^{\prime}+\mathfrak{L}^{\perp}\right)$.

Proof. In proving Proposition 5 we actually established a somewhat stronger result. 
(24) If $l \in P^{-1}\left(\mathcal{O}_{\chi}\right)$, there is a $k \in K$ such that $k \cdot l \in f^{\prime}+\mathfrak{f}^{\perp}$, where $k \cdot l=\operatorname{Ad}^{*}(k) l$. The natural actions of $\operatorname{Ad}^{*}(K)$ on $\mathfrak{g}^{*}$ and $\mathfrak{f}^{*}$ are equivariant under $P: \mathfrak{g}^{*} \rightarrow \mathfrak{l}^{*}$. Let

$$
\begin{aligned}
H & =\operatorname{Stab}_{K}(f)=\{x \in K: x \cdot f=f\} \\
& =\left\{x \in K: \operatorname{Ad}^{*}(x)\left(f^{\prime}+\mathfrak{H}^{\perp}\right) \subseteq\left(f^{\prime}+\mathfrak{H}^{\perp}\right)\right\} .
\end{aligned}
$$

This is a closed, connected subgroup of $K$ since $\operatorname{Ad}^{*}(K)$ acts unipotently on $\mathfrak{f}^{*}$. Let $\mathcal{O}$ be a $G$-orbit meeting $P^{-1}\left(\mathcal{O}_{\chi}\right)$. Then $\mathcal{O}$ meets $f^{\prime}+\mathfrak{H}^{\perp}$ and we may take a representative $l \in \mathcal{O} \cap\left(f^{\prime}+\mathfrak{H}^{\perp}\right)$. Of course $H \cdot l \subseteq K \cdot l \cap\left(f^{\prime}+\mathfrak{f}^{\perp}\right)$ and is a closed submanifold; but in fact, $H \cdot l=K \cdot l \cap\left(f^{\prime}+\mathfrak{H}^{\perp}\right)$ because

$$
k \cdot l \in f^{\prime}+\mathfrak{l}^{\perp} \Leftrightarrow k \cdot f=k \cdot P(l)=P(k \cdot l)=P\left(f^{\prime}+\mathfrak{l}^{\perp}\right)=f \Leftrightarrow k \in H .
$$

The case $\tau_{0}>0$ is covered by Theorem 3. If $\tau_{0}=0$, then for $\nu$-a.e. $l^{\prime} \in \Sigma^{x}$, $\mathcal{O}_{l^{\prime}}=G \cdot l^{\prime}$ meets $P^{-1}\left(\mathcal{O}_{\chi}\right)$ in a closed submanifold consisting of a finite number $(\leqslant B)$ of $\operatorname{Ad}^{*}(K)$-orbits, having representatives $l_{1}, \ldots, l_{q}$ in $f^{\prime}+\mathfrak{l}^{\perp}$. Now $K \cdot l_{j} \cap$ $\left(f^{\prime}+\mathfrak{f}^{\perp}\right)=H \cdot l_{j}$ is a closed submanifold in $f^{\prime}+\mathfrak{f}^{\perp}$, and these are the connected components of $G \cdot l^{\prime} \cap\left(f^{\prime}+\mathfrak{l}^{\perp}\right)$. Hence $m\left(l^{\prime}\right)=n_{1}\left(l^{\prime}\right)$ for $\nu$-a.e. $l \in \Sigma^{\chi}$, and we are done.

7. Here are examples illustrating the various theorems. For brevity, we adhere to the following procedure.

(1) The Lie algebra $g$ will be specified by giving a strong Mal'cev basis $X_{1}, \ldots, X_{n}$ $\left(g_{i}=\mathbf{R}\right.$-span $\left\{X_{1}, \ldots, X_{i}\right\}$ is an ideal), plus those nonzero brackets $\left[X_{i}, X_{j}\right]$ with $i>j$. The dual basis in $\mathrm{g}^{*}$ will be denoted $l_{1}, \ldots, l_{n}$.

(2) The Lie algebra $\mathfrak{f}$ will be spanned by some of the $X_{j}$, which will be named. We then give $\chi$. (We restrict attention to 1-dimensional $\chi$.)

(3) We shall then determine the set $T(e)$ of indices $j$ such that $l_{j}$ appears in the cross sectioning subspace $V_{T(e)}$ for each layer $U_{e}^{\sim}(e \in \mathscr{E})$, and describe $V_{T(e)} \cap U_{e}^{\sim}$.

Thereafter, we give the direct integral decomposition.

EXAmPLE 1. The 3-dimensional Heisenberg group, spanned by $Z=X_{1}, Y=X_{2}$, $X=X_{3}$, with $[X, Y]=Z$. Let $\mathfrak{f}=\mathbf{R}$-span $\{Y\}$ and $\chi_{\alpha}(\exp y Y)=e^{i \alpha y}$. Then

$$
\begin{aligned}
& T_{1}=\{1\}, \quad V_{T_{1}} \cap U_{e^{(1)}}=\mathbf{R}-\operatorname{span}\left\{l_{1}\right\} \backslash\{0\}, \\
& T_{2}=\{1,2,3\}, \quad V_{T_{2}} \cap U_{e^{(2)}}=\mathbf{R}-\operatorname{span}\left\{l_{2}, l_{3}\right\} .
\end{aligned}
$$

The generic orbits for $K \backslash G$ correspond to $T_{1}$; if $\lambda \neq 0$, the orbit for $\lambda l_{1}$ is $\lambda l_{1}+Z^{\perp}$, which is 2-dimensional. Each orbit intersects $f_{\alpha}^{\prime}+\mathfrak{H}=\alpha l_{2}+Y^{\perp}$ in a single line. Hence for any $\alpha, \Sigma^{\chi}=\left\{\lambda l_{1}: \lambda \neq 0\right\}$; furthermore, $\tau_{0}=0$ here, so

$$
\text { Ind }_{K \rightarrow G}\left(\chi_{\alpha}\right) \cong \int_{\mathbf{R} \backslash\{0\}}^{\oplus} \pi_{\lambda} d \lambda
$$

where $\pi_{\lambda}$ corresponds to $\lambda l_{1}$.

EXAMPLE 2. Let $g$ be spanned by $Z=X_{1}, Y=X_{2}, X=X_{3}$, and $W=X_{4}$, with $[W, X]=Y,[W, Y]=Z$. Let $1=\mathbf{R}-\operatorname{span}\{W\}$, and let $\chi_{\alpha}(\exp w W)=e^{i \alpha w}=$ $e^{i f_{\alpha}(w W)}$. There are three possible $T$ 's:

$$
\begin{aligned}
& T_{1}=\{1,3\}, \quad V_{T_{1}} \cap U_{e^{(1)}}=\mathbf{R}-\operatorname{span}\left\{l_{1}, l_{3}\right\} \backslash \mathbf{R}-\operatorname{span}\left\{l_{3}\right\}, \\
& T_{2}=\{1,2\}, \quad V_{T_{2}} \cap U_{e^{(2)}}=\mathbf{R}-\operatorname{span}\left\{l_{2}\right\} \backslash\{0\}, \\
& T_{3}=\{1,2,3,4\}, \quad V_{T_{3}} \cap U_{e^{(3)}}=\mathbf{R}-\operatorname{span}\left\{l_{3}, l_{4}\right\} .
\end{aligned}
$$


The generic orbits for $K \backslash G$ correspond to $T_{1}$; the $\operatorname{Ad}^{*}(K)$-orbit for $\lambda l_{1}+\mu l_{3}$ is

$$
\left\{\lambda l_{1}+t_{1} l_{2}+\left(\mu+t_{1}^{2} / 2 \lambda\right) l_{3}+t_{2} l_{4}: t_{1}, t_{2} \in \mathbf{R}\right\}=\mathcal{O}_{\lambda, \mu} .
$$

This meets $f_{\alpha}^{\prime}+\mathfrak{L}^{\perp}=\alpha l_{4}+W^{\perp}$ in a 1-dimensional manifold, the parabola parametrized by

$$
t_{1} \mapsto \lambda l_{1}+t_{1} l_{2}+\left(\mu+t_{1}^{2} / 2 \lambda\right) l_{3}+\alpha l_{4}, \quad\left(t_{1} \in \mathbf{R}\right) .
$$

Hence $\Sigma^{x}=\left\{\lambda l_{1}+\mu l_{3}: \lambda \neq 0\right\}$; since $\tau_{0}=0$ in this case, we get

$$
\operatorname{Ind}_{K \rightarrow G}(\chi)_{\alpha} \cong \int_{(\mathbf{R} \backslash\{0\}) \times \mathbf{R}}^{\oplus} \pi_{\lambda, \mu} d \lambda d \mu,
$$

where $\pi_{\lambda, \mu}$ corresponds to $\mathcal{O}_{\lambda, \mu}$.

If we let $\mathfrak{f}=\mathbf{R}$-span $\{Y\}$ and let $\chi_{\alpha}(\exp y Y)=e^{i \alpha y}$, the generic orbits again correspond to $T_{1}$; furthermore, $\mathcal{O}_{\lambda, \mu} \cap\left(\alpha l_{2}+Y^{\perp}\right)$ is again a line, so that

$$
\operatorname{Ind}_{K \rightarrow G}(\chi)_{\alpha} \cong \int_{(\mathbf{R} \backslash\{0\}) \times \mathbf{R}}^{\oplus} \pi_{\lambda, \mu} d \lambda d \mu .
$$

Now let $\mathfrak{f}=\mathbf{R}$-span $\{X\}$ and let $\chi_{\alpha}(\exp x X)=e^{i \alpha x}$. Then the generic orbits again correspond to $T_{1}$, but now $\mathcal{O}_{\lambda, \mu} \cap\left(f_{\alpha}^{\prime}+\mathfrak{l}^{\perp}\right)=\mathcal{O}_{\lambda, \mu} \cap\left(\alpha l_{3}+X^{\perp}\right)$ is

(i) empty, if $\lambda(\alpha-\mu)<0$;

(ii) a single line, if $\alpha=\mu$;

(iii) two lines, if $\lambda(\alpha-\mu)>0$.

The representations $\alpha=\mu$ give a set of measure 0 , and in this case $\tau_{0}=0$, so we get

$$
\operatorname{Ind}_{K \rightarrow G}\left(\chi_{\alpha}\right) \cong \int_{C_{\alpha}}^{\oplus} 2 \pi_{\lambda, \mu} d \lambda d \mu
$$

where $C_{\alpha}=\{(\lambda, \mu): \lambda \neq 0, \operatorname{sgn} \lambda=\operatorname{sgn}(\alpha-\mu)\}$.

Finally, let $f=\mathbf{R}$-span $\{Z\}$ and let $\chi_{\alpha}(\exp z Z)=e^{i \alpha z}$. There are now two cases. If $\alpha \neq 0$, then the generic orbits correspond to $T_{1} ;\left(\alpha l_{1}+Z^{\perp}\right) \cap \mathcal{O}_{\lambda, \mu}=\mathcal{O}_{\lambda, \mu}$ if $\alpha=\lambda$ and is empty otherwise. Again, $\tau_{0}=0$ and Theorem 3 says that

$$
\text { Ind }_{K \rightarrow G}\left(\chi_{\alpha}\right) \cong \int_{\mathbf{R}}^{\oplus} \infty \cdot \pi_{\alpha, \mu} d \mu .
$$

If $\alpha=0$, the generic orbits correspond to $T_{2}$; the orbit $\mathcal{O}_{\gamma}$ for $\gamma l_{2}(\gamma \neq 0)$ is $\gamma l_{2}+\{Z, Y\}^{\perp}$, and this orbit meets $Z^{\perp}$ in a plane. So

$$
\operatorname{Ind}_{K \rightarrow G}\left(\chi_{0}\right) \cong \int_{\mathbf{R} \backslash\{0\}}^{\oplus} \infty \cdot \pi_{\gamma} d \gamma,
$$

where $\pi_{\gamma}$ corresponds to $\mathcal{O}_{\gamma}$. (When $\alpha=0$, one is looking at the regular representation of $K \backslash G-K$ is normal here-and the result is not surprising.)

EXAMPLE 3. Let $g$ be the 6-dimensional Lie algebra spanned by $X, Y_{1}, Y_{2}, Y_{3}, Y_{4}, Y_{5}$, with $\left[X, Y_{j}\right]=Y_{j+1}(1 \leqslant j \leqslant 4),\left[X, Y_{5}\right]=0,\left[Y_{i}, Y_{j}\right]=0$. Let the dual basis in $g^{*}$ be $e, f_{1}, f_{2}, f_{3}, f_{4}, f_{5}$. The orbits in general position (those in the first layer $U_{e(1)}$ ) are parametrized by points $b=\left(b_{1}, b_{2}, b_{3}, b_{4}\right) \in \mathbf{R}^{4}$ with $b_{4} \neq 0$ :

$$
\begin{aligned}
\mathcal{O}_{b}=\left\{t e+\left(b_{1}+b_{2} x+b_{3} \frac{x^{2}}{2}+b_{4}\right.\right. & \left.\frac{x^{4}}{24}\right) f_{1}+\left(b_{2}+b_{3} x+b_{4} \frac{x^{3}}{6}\right) f_{2} \\
& \left.+\left(b_{3}+b_{4} \frac{x^{2}}{2}\right) f_{3}+\left(b_{4} x\right) f_{4}+b_{4} f_{5} ; t, x \in \mathbf{R}\right\} .
\end{aligned}
$$


Let $\mathfrak{f}=\mathbf{R}$-span $\left\{Y_{2}, Y_{3}\right\}$, and let $\sigma$ be the trivial representation on $\mathfrak{f}$. Then $\mathcal{O}_{b}$ meets $\mathfrak{f}^{\perp}$ if we can find $x$ with

$$
b_{3}+b_{4}\left(x^{2} / 2\right)=0, \quad b_{2}+b_{3} x+b_{4}\left(x^{3} / 6\right)=0 .
$$

So $b_{3}=-b_{4}\left(x^{2} / 2\right)$ and $b_{2}=-b_{4}\left(x^{3} / 3\right)$; that is, $x=-\left(3 b_{2} / b_{4}\right)^{1 / 3}$, and $b_{3}=$ $-\left(9 b_{2}^{2} b_{4}\right)^{1 / 3}$. That is, the representations appearing in $\rho$ correspond to a thin subset of the representations of $G^{\wedge}$ in general position-specifically, those with orbit representatives in the intersection of an algebraic variety with $V_{T_{1}} \cap U_{e(1)}=\{b \in$ $\left.\mathbf{R}^{4}: b_{4} \neq 0\right\}$. (They all have multiplicity 1 , as is easily checked.)

For an interesting variant, let $\mathfrak{f}=\mathbf{R}$-span $\left\{Y_{2}, Y_{3}, Y_{5}\right\}$; let $\sigma$ be the representation

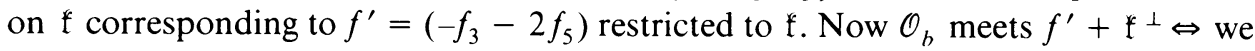
can solve

$$
b_{4}=-2, \quad b_{3}+b_{4}\left(x^{2} / 2\right)=-1, \quad b_{2}+b_{3} x+b_{4}\left(x^{3} / 6\right)=0
$$

for some $x$. Then $b_{3}=-1+x^{2}, x= \pm \sqrt{1+b_{3}}$, so that $9 b_{2}^{2}=\left(2 b_{3}-1\right)^{2}\left(1+b_{3}\right)$. Thus, in the cross-section $V_{T_{1}} \cap \tilde{U_{e(1)}}$, for orbits in general position, the set of representatives $\Sigma^{x}$ for orbits meeting $f^{\prime}+\mathfrak{f}^{\perp}$ is an algebraic surface with a singularity. In a stratification of $\Sigma^{\chi}$, the singularity is a union of lower dimensional manifolds, and hence does not contribute to the direct integral decomposition of $\rho$.

EXAMPLE 4. Let $\mathrm{g}$ be the quotient of the previous example by its center, $\mathbf{R} Y_{5}$; equivalently, $g$ is the Lie algebra spanned by $X, Y_{1}, Y_{2}, Y_{3}, Y_{4}$, with $\left[X, Y_{j}\right]=Y_{j+1}$, $1 \leqslant j \leqslant 3 ;\left[X, Y_{4}\right]=0,\left[Y_{i}, Y_{j}\right]=0$. Let the dual basis in $g^{*}$ be $e, f_{1}, f_{2}, f_{3}, f_{4}$. The orbits in general position (those in $\left.U_{e(1)}^{-}\right)$are parametrized by points $b=\left(b_{1}, b_{2}, b_{3}\right)$ $\in \mathbf{R}^{3}$ with $b_{3} \neq 0$ :

$$
\mathcal{O}_{h}=\left\{t e+\left(b_{1}+b_{2} \frac{x}{2}+b_{3} \frac{x^{3}}{6}\right) f_{1}+\left(b_{2}+b_{3} \frac{x^{2}}{2}\right) f_{3}+b_{3} f_{4}: t, x \in \mathbf{R}\right\} .
$$

Set $\mathfrak{k}=\mathbf{R}$-span $\left\{f_{1}\right\}$, and let $\boldsymbol{\sigma}=\chi_{\alpha}$ correspond to $\alpha f_{1}$ restricted to $\mathfrak{f}$. Then $\left(\alpha f^{\prime}+\mathfrak{t}^{\perp}\right) \cap \mathcal{O}_{h}$ is a finite union of lines, each line being an $\operatorname{Ad}^{*}(K)$-orbit. The number of lines is exactly the number of distinct real solutions to $b_{3} x^{3} / 6+b_{2} x / 2$ $+b_{1}=\alpha$. Let $b_{0}=b_{1}-\alpha$. The equation $b_{3} x^{3}+3 b_{2} x+6 b_{0}$ always has one or three real roots (counting multiplicities); its discriminant is $D=-108 b_{3}^{-3}\left(b_{2}^{3}+9 b_{0}^{2}\right)$, and the equation has three real roots if $D>0$, one real root if $D<0$, and two real roots (one repeated) if $D=0$; see pp. 178-180 of [15].

Thus $V_{T_{1}} \cap \tilde{U_{e(1)}}=\left\{\left(b_{0}, b_{2}, b_{3}\right): b_{i} \in \mathbf{R}, b_{3} \neq 0\right\}$ is equal to $\Sigma_{(1)}^{x}=$ representatives in this set whose orbits meet $f^{\prime}+\mathfrak{f}^{\perp}$. The multiplicity function $n\left(l^{\prime}\right)=$ number of $\operatorname{Ad}^{*}(K)$-orbits in $\mathcal{O}_{l^{\prime}} \cap\left(\alpha f_{1}+\mathfrak{f}^{\perp}\right)$ has values

$$
\begin{aligned}
& n=1 \text { on }\left\{\left(b_{0}, b_{2}, b_{3}\right): b_{3} \neq 0, b_{3}\left(b_{2}^{2}+9 b_{0}^{2}\right)>0\right\}, \\
& n=3 \text { on }\left\{\left(b_{0}, b_{2}, b_{3}\right): b_{3} \neq 0, b_{3}\left(b_{2}^{2}+9 b_{0}^{2}\right)<0\right\} ;
\end{aligned}
$$

$n=2$ on a lower dimensional set of $\nu$-measure zero. This shows that $\rho$ need not have uniform multiplicity.

By considering Lie algebras $g$ spanned by $X_{1}, \ldots, X_{n}$, with $\left[X_{n}, X_{j}\right]=X_{j-1}$ for $1<j<n$, and letting $\mathfrak{f}=\mathbf{R}$-span $\left\{X_{n-1}\right\}$, one can get an arbitrarily large finite number of different multiplicities in $\operatorname{Ind}_{K \rightarrow G}\left(\chi_{\alpha}\right)$. 
EXAMPLE 5 (“SyMmeTric SPACES"). Let $\mathfrak{g}$ be nilpotent (corresponding to $G$ ), and let $\theta: \mathfrak{g} \rightarrow \mathfrak{g}$ be an involutive automorphism. Denote the corresponding automorphism on $G$ by $\theta$, too. Let $\mathfrak{f}=\{X \in \mathfrak{g}: \theta X=X\}, \mathfrak{g}=\{X \in \mathfrak{g}: \theta X=-X\}$, $K=\exp \mathfrak{f}, S=\exp \mathfrak{s}$. Then $\mathfrak{f} \oplus \mathfrak{S}=\mathfrak{g}$ (as vector spaces), $\mathfrak{f}$ is a Lie subalgebra, $[\mathfrak{f}, \mathfrak{g}] \subseteq \mathfrak{g}$, and $[\mathfrak{g}, \mathfrak{g}] \subseteq \mathfrak{f}$. Moreover, $K=\{x \in G: \theta x=x\}$, and $G=K \cdot S=S$. $K$.

Let $\rho$ be the quasi-regular representation on $K \backslash G$. Benoist has shown in [1] that $\rho$ is multiplicity-free. This result also follows from our Theorem 6 . We need the following result.

Proposition 6. With $G, K, \theta$ as above, let $l, l^{\prime} \in \mathfrak{t}^{\perp}$ be such that $l^{\prime}=\operatorname{Ad}^{*}(x) l$ for some $x \in G$. Then $l^{\prime}=\operatorname{Ad}^{*}(k) l$ for some $k \in K$.

Proof. Write $x=s \cdot k$; then $l^{\prime}=\operatorname{ad}^{*}(s) l_{0}$, where $l_{0}=\operatorname{Ad}^{*}(k) l$. Let $s=\exp W$, $W \in \mathfrak{s}$; we shall prove first that $\operatorname{ad}^{*}(W)^{m} l_{0} \in \mathfrak{F}^{\perp}$, all $m \geqslant 0$. Note that

$$
\left(\left(\operatorname{ad}^{*} W\right)^{m} l_{0}\right)(Y)=l_{0}\left(\operatorname{ad}(-W)^{m} Y\right) ;
$$

if $Y \in \mathfrak{f}$, then $\operatorname{ad}(-W)^{m} Y \in \mathfrak{f}$ for all even $m$ (since $Y \in \mathfrak{f}$ ), so that $\operatorname{ad}^{*}(W)^{m} l_{0} \in \mathfrak{f}^{\perp}$ for all even $m$.

Now let $\mathfrak{k}_{j}=\left\{Y \in \mathfrak{f}: \operatorname{Ad}(-W)^{j} Y=0\right\}$. We shall prove that

$$
\text { For all } m \geqslant 0 \text { and all } j \geqslant 0, \operatorname{ad}^{*}(W)^{m} l_{0} \in \mathfrak{i}_{j}^{\perp} \text {. }
$$

The proof is by induction on $j$. As noted above, (29) holds for even $m$ and all $j$. Clearly (29) holds for all $m \geqslant j$, by definition of the $\mathfrak{f}_{j}$, hence (29) holds for all $m$ when $j=1$. (When $j=0,(29)$ is trivial.) Now assume that (29) is true for all even $m$ when $j<i$. Then (29) holds for all $m \geqslant 2$ when $j=i$ since

$$
\operatorname{ad}^{*}(W)^{m} l_{0}(Y)=\operatorname{ad}^{*}(W)^{m-2} l_{0}([W,[W, Y]]),
$$

and $[W,[W, Y]] \in \mathfrak{l}_{i-2}$. Moreover, $l_{0}(Y)=0$, since $l \in \mathfrak{l}^{\perp}$. That proves (29) for $j=i$ and $m \neq 1$. Finally, we have

$$
\begin{aligned}
0 & =\operatorname{Ad}^{*}(\exp W) l_{0} Y \quad \text { (by hypothesis) } \\
& =\sum_{m=0}^{\infty} \frac{1}{m !}\left(\operatorname{ad}^{*} W\right)^{m} l_{0}(Y)=\left(\operatorname{ad}^{*} W\right) l_{0}(Y),
\end{aligned}
$$

since we have shown that the other terms in the series are 0 ; thus (29) holds for all $m$ with $j=i$, as claimed.

Since $\mathfrak{f}_{j}=\mathfrak{f}$ when $\mathfrak{t}$ is large, we see that $\left(\operatorname{ad}^{*} W\right) l_{0}(Y)=0$ for $Y \in \mathfrak{f}$. But for $X \in \mathfrak{s}$,

$$
\left(\operatorname{ad}^{*} W\right) l_{0}(X)=l_{0}([X, W])=0,
$$

because $[X, W] \in \mathfrak{f}$. Thus $\left(\operatorname{ad}^{*} W\right) l_{0}=0$. It follows that

$$
l^{\prime}=\left(\operatorname{Ad}^{*} s\right) l_{0}=l_{0}=\left(\operatorname{Ad}^{*} k\right) l,
$$

which proves the proposition.

Proposition 6 shows that for every $l \in \mathfrak{f}^{\perp}, \operatorname{Ad}^{*}(G) l \cap \mathfrak{F}^{\perp}=\operatorname{Ad}^{*}(K) l$. Thus $\rho$ has multiplicity 1 , by Theorem 6 . 
EXAmple 6. In most examples so far, the decomposition of $\rho=\operatorname{Ind}_{K \rightarrow G}(\chi)$ involved only representations in general position-those in the first layer $U_{e^{(1)}}$. The exception was that part of Example 2 where $f^{f}$ contained the center of $G$. Here is one example in which representations not in general position occur and $K$ contains no central element except the identity.

Let $G$ be the $6 \times 6$ upper triangular matrices with 1's on the diagonal. Then $g$ is the Lie algebra of all $6 \times 6$ upper triangular matrices with diagonal zeros, and we may regard $g^{*}$ as the space of all $6 \times 6$ lower triangular matrices. Let $K=\exp { }^{*}$ where

$$
\mathfrak{f}=\left[\begin{array}{c|ccc}
* & * & 0 \\
* & * & 0 \\
0 & 0 & 0 \\
\hdashline 0 & & 0
\end{array}\right], \quad * \text { arbitrary real, }
$$

and let $\chi_{\alpha}$ be the trivial character. Then

$$
\mathfrak{f}^{\perp}=\left[\begin{array}{ccc:lll}
0 & 0 & 0 & & & \\
a_{21} & 0 & 0 & 0 & & \\
a_{31} & a_{32} & 0 & & & \\
\hdashline a_{41} & a_{42} & a_{43} & 0 & 0 & 0 \\
a_{51} & 0 & 0 & a_{54} & 0 & 0 \\
a_{61} & 0 & 0 & a_{64} & a_{65} & 0
\end{array}\right], \quad a_{i j} \text { real. }
$$

An element of $\mathrm{g}^{*}$ is in general position $\Leftrightarrow a_{61} \neq 0$ and $a_{51} a_{62}-a_{52} a_{61} \neq 0$ (see e.g [9]); thus $\mathfrak{f}^{\perp}$ misses all orbits in general position in $\mathfrak{g}^{*}$. We omit calculation of multiplicities in this example.

EXAMPLE 7. Whether Ind $_{K \rightarrow G}\left(\chi_{\alpha}\right)$ includes representations in general position may depend on the choice of $\mathrm{Mal}^{\prime}$ cev basis. One simple example is the following: let $g$ be spanned by $X_{1}, X_{2}, X_{3}, Y_{1}, Y_{2}, Y_{3}$ with the $Y_{i}$ central and $\left[X_{1}, X_{2}\right]=Y_{3}$, $\left[X_{2}, X_{3}\right]=Y_{1},\left[X_{3}, X_{1}\right]=Y_{2}$. Let $\mathfrak{f}=\mathbf{R}$-span $\left\{Y_{1}\right\}$ and let $\chi_{\alpha}$ be the trivial representation on $K$. If we order elements in the Mal'cev basis as $Y_{1}, Y_{2}, Y_{3}, X_{1}, X_{2}, X_{3}$ then the generic elements of $\mathrm{g}^{*}$ are those with $l\left(Y_{3}\right) \neq 0$. Hence $\mathfrak{f}^{\perp}$ meets the generic orbits in $\mathrm{g}^{*}$. But if the ordering is $Y_{1}, Y_{2}, Y_{3}, X_{2}, X_{3}, X_{1}$ then the generic elements have $l\left(Y_{1}\right) \neq 0$ and $\mathfrak{f}^{\perp}$ does not meet the generic orbits.

EXAMPLE 8. If $G$ is any nilpotent Lie group and $K$ is connected, normal then the multiplicities can only be 1 (multiplicity free) or $\infty$. This was first established in [7], by other arguments. We want to show that it follows easily from Theorems 5,6 . It suffices to show that orbit intersections $\mathcal{O}_{l} \cap\left(f^{\prime}+\mathfrak{f}^{\perp}\right)$ are always connected.

Pick an $f \in \mathcal{O}_{\chi} \subseteq \mathfrak{f}^{*}$, let $f^{\prime}$ be any extension to $\mathfrak{g}$, let $P: \mathfrak{g}^{*} \rightarrow \mathfrak{l}^{*}$ be the natural projection, and write $X=P^{-1}\left(\mathcal{O}_{\chi}\right), Y=P^{-1}\{f\}=f^{\prime}+\mathfrak{H}^{\perp}$ for brevity. Since the natural actions of $\mathrm{Ad}^{*}(G)$ on $\mathrm{g}^{*}$ and $\mathfrak{F}^{*}$ are equivariant under $P$, any orbit $\mathcal{O}_{1}$ meeting $X$ also meets $Y$. Let $H=\operatorname{Stab}_{G}(f)=\left\{x \in G: \operatorname{Ad}^{*}(x) Y=Y\right]$; it is a closed, connected subgroup since $\operatorname{Ad}^{*}(G)$ acts unipotently on $\mathfrak{f}^{*}$. 
Fix an $l \in Y$. For $x \in G$ write $x \cdot l=\operatorname{Ad}^{*}(x) l$. Then $x \cdot l \in Y \Leftrightarrow f=P(l)=$ $P(x \cdot l)=x \cdot f \Leftrightarrow x \in H$, hence $\mathcal{O}, \cap Y=H \cdot l$ is a closed connected manifold. We now claim that $\mathcal{O}_{l} \cap X=K \cdot(H \cdot l)$, and hence is connected. In fact, if $x \cdot l \in X$, then $x \cdot l \in \mathcal{O}, \cap X$, so there is a $k \in K$ such that $k x \cdot l \in \mathcal{O}_{l} \cap Y=S \cdot l$, and we see that $\mathcal{O}_{l} \cap X \subseteq K S \cdot l$. The converse inclusion is obvious, so the intersection is always connected.

\section{BIBLIOGRAPHY}

1. Y. Benoist, Espaces symmetriques exponentielles, Thesis III ${ }^{\text {me }}$ cycle, Paris VII, 1983.

2. I. K. Busiyatskaya, Representations of exponential Lie groups, J. Funct. Anal. Appl. 7 (1973), 151-152. (Russian)

3. L. Corwin, A representation-theoretic criterion for local solvability of left invariant differential operators on nilpotent Lie groups, Trans. Amer. Math. Soc. 264 (1981), 113-120.

4. L. Corwin and F. P. Greenleaf, Character formulas and spectra of compact nilmanifolds, J. Funct. Anal. 21 (1976), 123-154.

5. J. Fox, On the spectrum of compact nilmanifolds, preprint, 1984.

6. E. A. Gorin, Asymptotic properties of polynomials and algebraic functions of several variables, Uspekhi Mat. Nauk 16 (1961), 93-119.

7. G. Grelaud, Desintegration de representations induites d' un groupe de Lie resoluble exponentiel, C. R. Acad. Sci. Paris, Ser. A, 277 (1973), 327-330.

8. Sur les representations des groupes de Lie resoluble, Thesis III $^{\mathrm{me}}$ cycle, Univ. de Poitiers, October, 1984.

9. A. A. Kirillov, Unitary representations of nilpotent Lie groups, Uspekhi Mat. Nauk 17 (1962), 57-110.

10. L. Pukanszky, Unitary representations of solvable Lie groups, Ann. Sci. Ecole Norm. Sup. 4 (1971), 457-608.

11. J. T. Schwartz, Differential geometry and topology, Gordon and Breach, New York, 1968.

12. A. Seidenberg, A new decision method for elementary algebra, Ann. of Math. (2) 60 (1954), 365-374.

13. H. Sussman, Analytic stratifications and subanalytic sets, monograph (in preparation).

14. A. Tarski, $A$ decision method for elementary algebra and geometry, 2nd ed., Univ. of California Press, Berkeley, 1951, $63 \mathrm{pp}$.

15. B. Van der Waerden, Modern algebra, 2nd ed., Ungar, New York, 1949.

Department of Mathematics, Rutgers University, New Brunswick, NeW Jersey 08903

Courant Institute of Mathematical Sciences, New York University, New York, New York 10012

Departement de Mathematique, Universite de Poitiers, 40 Avenue Recteur Pineau, 86022 Poitiers, France 